\title{
COMBINATORICS OF THE DOUBLE-DIMER MODEL
}

\author{
HELEN JENNE
}

\begin{abstract}
We prove that the partition function for tripartite double-dimer configurations of a planar bipartite graph satisfies a recurrence related to the Desnanot-Jacobi identity from linear algebra. A similar identity for the dimer partition function was established nearly 20 years ago by Kuo and has applications to random tiling theory and the theory of cluster algebras. This work was motivated in part by the potential for applications in these areas. Additionally, we discuss an application to a problem in Donaldson-Thomas and Pandharipande-Thomas theory. The proof of our recurrence requires generalizing work of Kenyon and Wilson; specifically, lifting their assumption that the nodes of the graph are black and odd or white and even.
\end{abstract}

\section{INTRODUCTION}

Let $G=\left(V_{1}, V_{2}, E\right)$ be a finite edge-weighted bipartite planar graph embedded in the plane with $\left|V_{1}\right|=\left|V_{2}\right|$. Let $\mathbf{N}$ denote a set of special vertices called nodes on the outer face of $G$ numbered consecutively in counterclockwise order. A doubledimer configuration on $(G, \mathbf{N})$ is a multiset of the edges of $G$ with the property that each internal vertex is the endpoint of exactly two edges, and each vertex in $\mathbf{N}$ is the endpoint of exactly one edge. In other words, it is a configuration of paths connecting the nodes in pairs, doubled edges, and disjoint cycles of length greater than two (called loops). Define a probability measure Pr where the probability of a configuration is proportional to the product of its edge weights times $2^{\ell}$, where $\ell$ is the number of loops in the configuration. Kenyon and Wilson initiated the study of the double-dimer model in [KW11a], when they showed how to compute the probability that a random double-dimer configuration has a particular

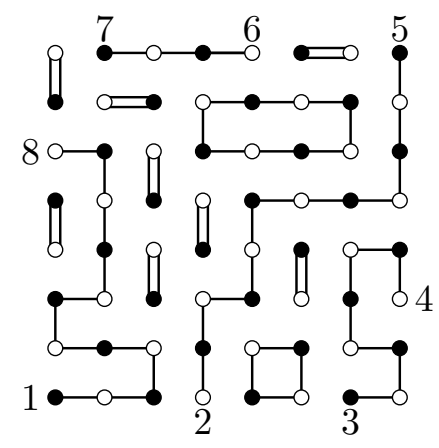

Figure 1. A double-dimer configuration on a grid graph with 8 nodes. In this configuration, the pairing of the nodes is $((1,8),(3,4),(5,2),(7,6))$. node pairing.

Before going into the details of Kenyon and Wilson's work, we will describe Kuo's recurrence for dimer configurations, which is the motivation for this paper, and state one of our main results. A dimer configuration (or perfect matching) of $G$ is a collection of the edges that covers all of the vertices exactly once. The weight of a dimer configuration is the product of its edge weights. Let $Z^{D}(G)$ denote the sum of the weights of all possible dimer configurations on $G$. In [Kuo04], Kuo proved that $Z^{D}(G)$ satisfies an elegant recurrence.

Theorem 1.0.1. [Kuo04, Theorem 5.1] Let $G=\left(V_{1}, V_{2}, E\right)$ be a planar bipartite graph with a given planar embedding in which $\left|V_{1}\right|=\left|V_{2}\right|$. Let vertices $a, b, c$, and d appear in a cyclic order on a face of $G$. If $a, c \in V_{1}$ and $b, d \in V_{2}$, then

$$
Z^{D}(G) Z^{D}(G-\{a, b, c, d\})=Z^{D}(G-\{a, b\}) Z^{D}(G-\{c, d\})+Z^{D}(G-\{a, d\}) Z^{D}(G-\{b, c\}) .
$$

His proof uses a technique called graphical condensation, which is named for its resemblance to Dodgson condensation, a method for computing the determinants of square matrices. 

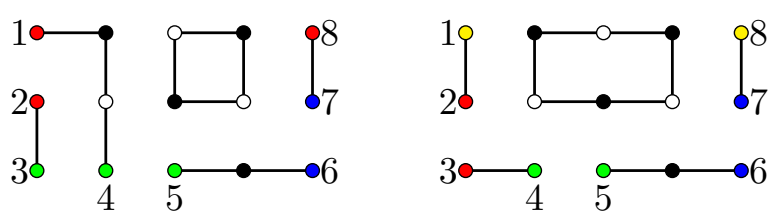

Figure 2. Two double-dimer configurations on a grid graph. The pairing of the nodes on the left is a tripartite pairing because the nodes can be colored contiguously using three colors so that no pair contains nodes of the same RGB color. The pairing on the right is not a tripartite pairing because four colors are required.
In this paper, we will show that when $\sigma$ is a tripartite pairing, a similar identity to (1.0.1) holds for $Z_{\sigma}^{D D}(G, \mathbf{N})$, the weighted sum of all double-dimer configurations on $(G, \mathbf{N})$ with pairing $\sigma$.

A planar pairing $\sigma$ is a tripartite pairing if the nodes can be divided into three circularly contiguous sets $R, G$, and $B$ so that no node is paired with a node in the same set (see Figure 2). We often color the nodes in the sets red, green, and blue, in which case $\sigma$ is the unique planar pairing in which like colors are not paired.

The following double-dimer version of equation (1.0.1) is a corollary to Theorem 1.3.3 in Section 1.3 .

Theorem 1.0.2. Let $G=\left(V_{1}, V_{2}, E\right)$ be a finite edge-weighted planar bipartite graph with a set of nodes N. Divide the nodes into three circularly contiguous sets $R, G$, and $B$ such that $|R|,|G|$ and $|B|$ satisfy the triangle inequality and let $\sigma$ be the corresponding tripartite pairing ${ }^{1}$. Let $x, y, w, v$ be nodes appearing in a cyclic order such that the set $\{x, y, w, v\}$ contains at least one node of each $R G B$ color $^{2}$. If $x, w \in V_{1}$ and $y, v \in V_{2}$ then

$$
\begin{aligned}
Z_{\sigma}^{D D}(G, \mathbf{N}) Z_{\sigma_{x y w v}}^{D D}(G, \mathbf{N}-\{x, y, w, v\})= & Z_{\sigma_{x y}}^{D D}(G, \mathbf{N}-\{x, y\}) Z_{\sigma_{w v}}^{D D}(G, \mathbf{N}-\{w, v\}) \\
& +Z_{\sigma_{x v}}^{D D}(G, \mathbf{N}-\{x, v\}) Z_{\sigma_{w y}}^{D D}(G, \mathbf{N}-\{w, y\}),
\end{aligned}
$$

where for $i, j \in\{x, y, w, v\}, \sigma_{i j}$ is the unique planar pairing on $\mathbf{N}-\{i, j\}$ in which like colors are not paired together.

We illustrate Theorem 1.0.2 with an example.

Example 1.0.3. If $G$ is a graph with eight nodes colored red, green, and blue as shown below, then $\sigma=((1,8),(3,4),(5,2),(7,6))$. If $x=8, y=1, w=2, v=5$, then by Theorem 1.0.2,

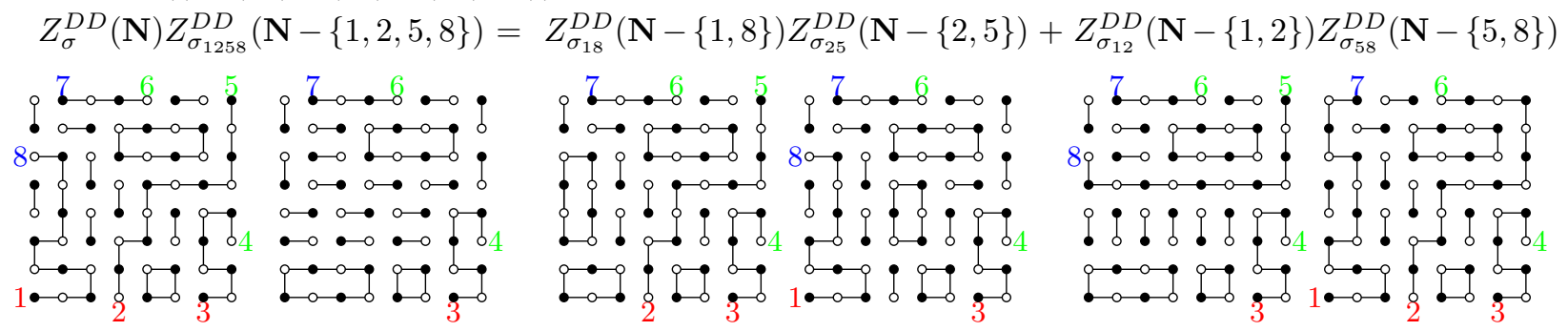

We were motivated to find an analogue of Theorem 1.0.1 by its potential applications, which we discuss in the next section.

1.1. Applications. Kuo's work has a variety of applications. For example, Kuo uses graphical condensation to give a new proof that the number of tilings of an order- $n$ Aztec diamond is $2^{n(n+1) / 2}$ [Kuo04, Theorem 3.2] and a new proof for MacMahon's generating function for plane partitions that are subsets of a box [Kuo04, Theorem 6.1]. His results also have applications to random tiling theory (see [Kuo04, Section 4.1]) and the theory of cluster algebras.

\footnotetext{
${ }^{1}$ If $|R|,|G|$, and $|B|$ do not satisfy the triangle inequality, there is no corresponding tripartite pairing $\sigma$.

${ }^{2}$ The nodes of $G$ have two colors: the black-white coloring from the bipartite assumption, and the RGB coloring. The coloring we are referring to is often clear from context, but to avoid ambiguity we will often write RGB color to emphasize that we are referring to the red, green, blue coloring of the nodes rather than the black-white coloring.
} 
Cluster algebras are a class of commutative rings introduced by Fomin and Zelevinsky [FZ02] to study total positivity and dual canonical bases in Lie theory. The theory of cluster algebras has since been connected to many areas of math, including quiver representations, Teichmüller theory, Poisson geometry, and integrable systems [Wil14]. In [LM17, LM20], Tri Lai and Gregg Musiker study toric cluster variables for the quiver associated to the cone over the del Pezzo surface $d P_{3}$, giving algebraic formulas for these cluster variables as Laurent polynomials. Using identities similar to Kuo's Theorem 1.0.1, they give combinatorial interpretations of most of these formulas [LM17].

We expect Theorem 1.0.2 to have similar applications. In addition, by using both Theorem 1.0.1 and Theorem 1.0.2 we can give a direct proof of a problem in Donaldson-Thomas and Pandharipande-Thomas theory.

1.1.1. Application to Donaldson-Thomas and Pandharipande-Thomas theory. Donaldson-Thomas (DT) theory, Pandharipande-Thomas (PT), and Gromov-Witten (GW) theory are branches of enumerative geometry closely related to mirror symmetry and string theory. The DT and GW theories give frameworks for counting curves $^{3}$ on a threefold $X$. One of the conjectures in [MNOP06a, MNOP06b] gives a correspondence between the DT and GW frameworks, which has been proven in special cases, such as when $X$ is toric [MOOP11].

PT theory gives a third framework for counting curves when $X$ is a nonsingular projective threefold that is Calabi-Yau. The correspondence between the DT and PT frameworks was first conjectured in [PT09b] and was proven in [Bri11], which is closely related to the work in [Tod10]. Specifically, let $X$ be a toric Calabi-Yau 3-fold. Define $Z_{D T}(q)=\sum_{n} I_{n} q^{n}$, where $I_{n}$ counts length $n$ subschemes of $X$, and $Z_{P T}(q)=\sum_{n} P_{n} q^{n}$, where $P_{n}$ counts stable pairs on $X$ (see [PT09b]). Bridgeland proved that these generating functions coincide up to a factor of $M(q)=\prod_{n=1}^{\infty} \frac{1}{\left(1-q^{n}\right)^{n}}$, which is the total $q$-weight of all plane partitions [Mac16].

Theorem 1.1.1. [Bri11, Theorem 1.1] $Z_{D T}(q)=Z_{P T}(q) M(q)^{4}$.

The application of Theorem 1.0.2 that we describe relates to Theorem 1.1.1 at the level of the topological vertex. Define $V_{\lambda, \mu, \nu}=q^{c(\lambda, \mu, \nu)} \sum_{\pi} q^{|\pi|}$, where the sum is taken over all plane partitions $\pi$ asymptotic to $(\lambda, \mu, \nu)$. Maulik, Nekrasov, Okounkov, and Pandharipande [MNOP06a, MNOP06b] proved that $Z_{D T}(q)=V_{\lambda, \mu, \nu}$ and thus $V_{\lambda, \mu, \nu}$ is called the DT topological vertex. Let $W_{\lambda, \mu, \nu}=$ $q^{c(\lambda, \mu, \nu)} \sum_{i} d_{i} q^{i}$ where $d_{i}$ is a certain weighted enumeration of labelled box configurations of length $i$ [PT09a]. In [PT09a, Theorem/Conjecture 2] Pandharipande and Thomas conjecture that $W_{\lambda, \mu, \nu}$ is the stable pairs vertex, i.e. that $Z_{P T}(q)=W_{\lambda, \mu, \nu}$.

In a forthcoming paper with Gautam Webb and Ben Young (for an extended abstract, see [JWY20]), we prove that

Conjecture 1.1.2. [PT09a, Calabi-Yau case of Conjecture 4] $V_{\lambda, \mu, \nu}=W_{\lambda, \mu, \nu} M(q)$.

Pandharipande and Thomas remark that a straightforward (but long) approach to this conjecture using DT theory exists [PT09a]. Our proof interprets $V_{\lambda, \mu, \nu}$ using the dimer model and $W_{\lambda, \mu, \nu}$ using the double-dimer model, and then uses Theorems 1.0.1 and 1.0.2 to show that both $V_{\lambda, \mu, \nu} / M(q)$ and $W_{\lambda, \mu, \nu}$ satisfy the same recurrence. Conjecture 1.1.2, taken together with a substantial body of geometric work, proves the aforementioned Theorem/Conjecture 2 of [PT09a]. For further details, see [JWY20].

\footnotetext{
${ }^{3}$ The frameworks differ in what is meant by a curve on $X$.

${ }^{4}$ In [Bri11, PT09a, MNOP06a, MOOP11] and elsewhere in the geometry literature, the formulas have $-q$ rather than $q$. The sign is there for geometric reasons which are immaterial to us.
} 
1.2. Proof of Theorem 1.0.2. Presently, we discuss the main ideas behind the proof of Theorem 1.0.2. We start by giving an overview of the results from [KW11a, KW09] that are needed for our work.

1.2.1. Background. Kenyon and Wilson gave explicit formulas for the probability that a random double-dimer configuration has a particular node pairing $\sigma$. When $\sigma$ is a tripartite pairing, this probability is proportional to the determinant of a matrix.

To be more precise, we need to introduce some notation and definitions. Since $G$ is bipartite, we can color its vertices black and white so that each edge connects a black vertex to a white vertex. Let $G^{B W}$ be the subgraph of $G$ formed by deleting the nodes except for the ones that are black and odd or white and even. Define $G^{W B}$ analogously, but with the roles of black and white reversed. Let $G_{i, j}^{B W}$ be the graph $G^{B W}$ with nodes $i$ and $j$ included if and only if they were not included in $G^{B W}$. For convenience, Kenyon and Wilson assume the nodes alternate in color, so all nodes are black and odd or white and even. (If a graph $G$ does not have this property, we can add edges of weight 1 to each node that has the wrong color to obtain a graph whose double-dimer configurations are in a one-to-one weight-preserving correspondence with double-dimer configurations of $G$.)

For each planar pairing $\sigma$, Kenyon and Wilson showed the normalized probability

$$
\widehat{\operatorname{Pr}}(\sigma):=\operatorname{Pr}(\sigma) \frac{Z^{D}\left(G^{W B}\right)}{Z^{D}\left(G^{B W}\right)}=\frac{Z_{\sigma}^{D D}(G, \mathbf{N})}{\left(Z^{D}\left(G^{B W}\right)\right)^{2}}
$$

that a random double-dimer configuration has pairing $\sigma$ is an integer-coefficient homogeneous polynomial in the quantities $X_{i, j}:=\frac{Z^{D}\left(G_{i, j}^{B W}\right)}{Z^{D}\left(G^{B W}\right)}$ [KW11a, Theorem 1.3].

For example, the normalized probability $\widehat{\operatorname{Pr}}$ that a random double-dimer configuration on eight nodes has the pairing $((1,8),(3,4),(5,2),(7,6))$ (see Figure 1$)$ is

$$
\begin{aligned}
\widehat{\operatorname{Pr}}\left(\begin{array}{l|l|l|l}
1 & 3 & 5 & 7 \\
8 & 4 & 2 & 6
\end{array}\right)= & X_{1,8} X_{3,4} X_{5,2} X_{7,6}-X_{1,4} X_{3,8} X_{5,2} X_{7,6}+X_{1,6} X_{3,4} X_{5,8} X_{7,2}-X_{1,8} X_{3,6} X_{5,2} X_{7,4} \\
& -X_{1,4} X_{3,6} X_{5,8} X_{7,2}+X_{1,6} X_{3,8} X_{5,2} X_{7,4} .
\end{aligned}
$$

Kenyon and Wilson gave an explicit method for computing these polynomials: they defined a matrix $\mathcal{P}^{(D D)}$ with rows indexed by planar pairings and columns indexed by odd-even pairings. They showed how to calculate the columns of the matrix completely combinatorially and proved that for any planar pairing $\sigma$,

$$
\widehat{\operatorname{Pr}}(\sigma)=\sum_{\text {odd-even pairings } \tau} \mathcal{P}_{\sigma, \tau}^{(D D)} X_{\tau}^{\prime}
$$

where $X_{\tau}^{\prime}=(-1)^{\# \text { crosses of } \tau} \prod_{i \text { odd }} X_{i, \tau(i)}$ [KW11a, Theorem 1.4].

In the case where $\sigma$ is a tripartite pairing, $\widehat{\operatorname{Pr}}(\sigma)$ is a determinant of a matrix whose entries are $X_{i, j}$ or 0 .

Theorem 1.2.1. [KW09, Theorem 6.1] Suppose that the nodes are contiguously colored red, green, and blue (a color may occur zero times), and that $\sigma$ is the (unique) planar pairing in which like colors are not paired together. Let $\sigma(i)$ denote the item that $\sigma$ pairs with item $i$. We have

$$
\widehat{\operatorname{Pr}}(\sigma)=\operatorname{det}\left[1_{i, j} \text { RGB-colored differently } X_{i, j}\right]_{j=\sigma(1), \sigma(3), \ldots, \sigma(2 n-1)}^{i=1,3,2 n-1} .
$$

Initially, it seems that Theorem 1.0.2 will follow immediately from combining Theorem 1.2.1 with the Desnanot-Jacobi identity. 
Theorem 1.2.2 (Desnanot-Jacobi identity). Let $M=\left(m_{i, j}\right)_{i, j=1}^{n}$ be a square matrix, and for each $1 \leq i, j \leq n$, let $M_{i}^{j}$ be the matrix that results from $M$ by deleting the ith row and the $j$ th column. Then

$$
\operatorname{det}(M) \operatorname{det}\left(M_{i, j}^{i, j}\right)=\operatorname{det}\left(M_{i}^{i}\right) \operatorname{det}\left(M_{j}^{j}\right)-\operatorname{det}\left(M_{i}^{j}\right) \operatorname{det}\left(M_{j}^{i}\right)
$$

However, we run into some technical obstacles, which we illustrate with an example.

1.2.2. Example. Suppose we wish to prove the equation from Example 1.0.3:

$Z_{\sigma}^{D D}(\mathbf{N}) Z_{\sigma_{1258}}^{D D}(\mathbf{N}-\{1,2,5,8\})=Z_{\sigma_{18}}^{D D}(\mathbf{N}-\{1,8\}) Z_{\sigma_{25}}^{D D}(\mathbf{N}-\{2,5\})+Z_{\sigma_{12}}^{D D}(\mathbf{N}-\{1,2\}) Z_{\sigma_{58}}^{D D}(\mathbf{N}-\{5,8\})$ where recall that $\sigma=((1,8),(3,4),(5,2),(7,6))$. Then the matrix $M$ from Theorem 1.2.1 is

$$
M=\left(\begin{array}{cccc}
X_{1,8} & X_{1,4} & 0 & X_{1,6} \\
X_{3,8} & X_{3,4} & 0 & X_{3,6} \\
X_{5,8} & 0 & X_{5,2} & 0 \\
0 & X_{7,4} & X_{7,2} & X_{7,6}
\end{array}\right) .
$$

Since the first row and column of $M$ correspond to nodes 1 and 8, respectively, and the third row and column correspond to nodes 5 and 2, we apply the Desnanot-Jacobi identity with $i=1$ and $j=3$ :

$$
\operatorname{det}(M) \operatorname{det}\left(M_{1,3}^{1,3}\right)=\operatorname{det}\left(M_{1}^{1}\right) \operatorname{det}\left(M_{3}^{3}\right)-\operatorname{det}\left(M_{1}^{3}\right) \operatorname{det}\left(M_{3}^{1}\right) .
$$

By Theorem 1.2.1,

$$
\operatorname{det}(M)=\frac{Z_{\sigma}^{D D}(G, \mathbf{N})}{\left(Z^{D}\left(G^{B W}\right)\right)^{2}}
$$

We also need to prove, for example, that

$$
\operatorname{det}\left(M_{3}^{3}\right)=\frac{Z_{\sigma_{25}}^{D D}(G, \mathbf{N}-\{2,5\})}{\left(Z^{D}\left(G^{B W}\right)\right)^{2}}
$$

where

$$
M_{3}^{3}=\left(\begin{array}{ccc}
X_{1,8} & X_{1,4} & X_{1,6} \\
X_{3,8} & X_{3,4} & X_{3,6} \\
0 & X_{7,4} & X_{7,6}
\end{array}\right) .
$$

An example of a double-dimer configuration counted by $Z_{\sigma_{25}}^{D D}(G, \mathbf{N}-\{2,5\})$ is shown in Figure 3.
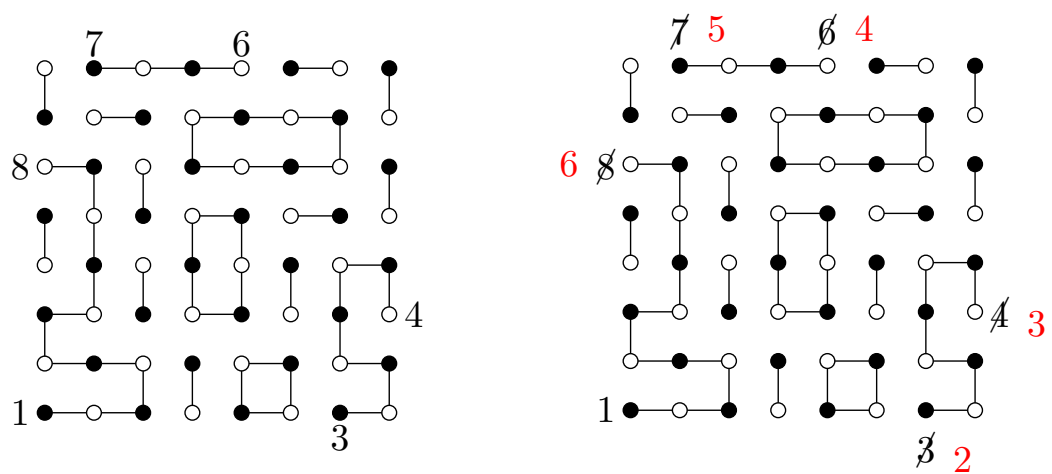

Figure 3. Left: A double-dimer configuration on a grid graph with node set $\mathbf{N}-\{2,5\}$ and pairing $((1,8),(3,4),(7,6))$. Right: The same double-dimer configuration after relabeling the nodes.

We cannot apply Theorem 1.2.1 to prove equation (1.2.2) because the nodes are not numbered consecutively. We might hope to resolve this by relabeling the nodes, as shown in Figure 3. But since Kenyon and Wilson assume that all nodes are black and odd or white and even, in order to 
satisfy the assumptions of Kenyon and Wilson's theorem, we need to add edges of weight 1 to nodes 2 and 3. Call the resulting graph $\widetilde{G}$ and let $\widetilde{X}_{i, j}=\frac{Z^{D}\left(\widetilde{G}_{i, j}^{B W}\right)}{Z^{D}\left(\widetilde{G}^{B W}\right)}$. The matrix from Theorem 1.2.1 is

$$
\widetilde{M}=\left(\begin{array}{ccc}
\widetilde{X}_{1,6} & 0 & \widetilde{X}_{1,4} \\
\widetilde{X}_{3,6} & \widetilde{X}_{3,2} & 0 \\
0 & \widetilde{X}_{5,2} & \widetilde{X}_{5,4}
\end{array}\right) .
$$

To prove equation (1.2.2) it suffices to show

$$
\left(Z^{D}\left(\widetilde{G}^{B W}\right)\right)^{2} \operatorname{det}(\widetilde{M})=\left(Z^{D}\left(G^{B W}\right)\right)^{2} \operatorname{det}\left(M_{3}^{3}\right),
$$

since $\operatorname{det}(\widetilde{M})=\frac{Z_{\sigma_{2}}^{D D}(\widetilde{G}, \mathbf{N}-\{2,5\})}{\left(Z^{D}\left(\widetilde{G}^{B W}\right)\right)^{2}}$ by Theorem 1.2.1.

Verifying equation (1.2.3) is a straightforward computation, but as we consider graphs with more nodes, the computations quickly become more involved. To be able to interpret the minors of Kenyon and Wilson's matrix outside of small examples, we need to lift their assumption that the nodes of the graph are black and odd or white and even.

Notice that under the assumption that the nodes of the graph are black and odd or white and even, $X_{i, j}=\frac{Z^{D}\left(G_{i, j}^{B W}\right)}{Z^{D}\left(G^{B W}\right)}=\frac{Z^{D}\left(G_{i, j}\right)}{Z^{D}(G)}$. This suggests that the correct generalization of Kenyon and Wilson's matrix will have entries $\frac{Z^{D}\left(G_{i, j}\right)}{Z^{D}(G)}$.

1.2.3. Our approach. The previous remark motivates our approach, which is to define $Y_{i, j}:=$ $\frac{Z^{D}\left(G_{i, j}\right)}{Z^{D}(G)}$ and $\widetilde{\operatorname{Pr}}(\sigma)=\frac{Z_{\sigma}^{D D}(G, \mathbf{N})}{\left(Z^{D}(G)\right)^{2}}$. When $G$ is a graph with nodes that are either black and odd or white and even, $Z^{D}(G)=Z^{D}\left(G^{B W}\right)$, so $Y_{i, j}=X_{i, j}$ and $\widetilde{\operatorname{Pr}}(\sigma)=\widehat{\operatorname{Pr}}(\sigma)$.

In this paper, we will prove analogues of many of Kenyon and Wilson's results from [KW11a, KW09] in the variables $Y_{i, j}$. Once we have established our generalization of Theorem 1.2.1, we will be able to apply the Desnanot-Jacobi identity to prove Theorem 1.0.2.

1.3. Organization of paper. This paper is structured as follows.

In Section 2, we generalize some of Kenyon and Wilson's results from [KW11a]. The main result of Section 2 is an analogue of [KW11a, Theorem 1.4]: we show that we can write $\widetilde{\operatorname{Pr}}(\sigma)$ as an integer-coefficient homogeneous polynomial in the quantities $Y_{i, j}$. To this end, we define

$$
Y_{\rho}^{\prime}=(-1)^{\# \text { crosses of } \rho} \prod_{i \text { black }} Y_{i, \rho(i)}
$$

for any black-white pairing $\rho$. Note that we work with black-white pairings rather than odd-even pairings since we are not requiring that the nodes are either black and odd or white and even. In [KW11a, KW09], black-white pairings and odd-even pairings coincide, so $X_{i, j}=0$ when $i$ and $j$ have the same parity, which occurs exactly when they have the same color ${ }^{5}$. In our general setting, $Y_{i, j}$ may be nonzero when $i$ and $j$ have the same parity, but if $i$ and $j$ are the same color then there are no dimer configurations of $G_{i, j}$, so $Y_{i, j}=0$.

Our analogue of Kenyon and Wilson's matrix $\mathcal{P}^{(D D)}$ (see equation $(1.2 .1)$ ) is $\mathcal{Q}^{(D D)}$. The rows of $\mathcal{Q}^{(D D)}$ are indexed by planar pairings and columns are indexed by black-white pairings. To prove that $\mathcal{Q}^{(D D)}$ is integer-valued, we show that the columns of this matrix can be computed combinatorially, and in Section 2.5 we prove the following theorem:

\footnotetext{
${ }^{5}$ Here, and elsewhere in Section 2, "same color" refers to the black-white coloring from the bipartite assumption.
} 
Theorem 1.3.1. Let $G$ be a finite edge-weighted planar bipartite graph with a set of nodes. For any planar pairing $\sigma$,

$$
\widetilde{\operatorname{Pr}}(\sigma)=\sum_{\text {black-white pairings } \rho} \mathcal{Q}_{\sigma, \rho}^{(D D)} Y_{\rho}^{\prime}
$$

where the coefficients $\mathcal{Q}_{\sigma, \rho}^{(D D)}$ are all integers.

To prove Theorem 1.3.1, we use Kenyon and Wilson [KW11a] as a road map, proving analogues of Lemmas 3.1 - 3.5 and Theorem 3.6 from [KW11a]. Because we follow their work so closely, before presenting each of our lemmas we state the corresponding lemma from [KW11a]. In some cases the proofs are very similar. In others, substantially more work is required.

In Section 3, we use our results from Section 2 to generalize Kenyon and Wilson's determinant formula from Theorem 1.2.1. Before stating our version of their formula, we observe that

$\operatorname{det}\left[1_{i, j} \text { RGB-colored differently } X_{i, j}\right]_{j=\sigma(1), \sigma(3), \ldots, \sigma(2 n-1)}^{i=1,3, \ldots, 2 n-1}=\operatorname{sign}_{O E}(\sigma) \operatorname{det}\left[1_{i, j} \text { RGB-colored differently } X_{i, j}\right]_{j=2,4, \ldots, 2 n}^{i=1,3, \ldots, 2 n-1}$,

where $\operatorname{sign}_{O E}(\sigma)$ is the parity of the permutation $\left(\begin{array}{llll}\frac{\sigma(1)}{2} & \frac{\sigma(3)}{2} & \cdots & \frac{\sigma(2 n-1)}{2}\end{array}\right)$ written in one-line notation.

We prove that

Theorem 1.3.2. Let $G$ be a finite edge-weighted planar bipartite graph with a set of nodes. Suppose that the nodes are contiguously colored red, green, and blue (a color may occur zero times), and that $\sigma$ is the (unique) planar pairing in which like colors are not paired together. We have

$$
\widetilde{\operatorname{Pr}}(\sigma)=\operatorname{sign}_{O E}(\sigma) \operatorname{det}\left[1_{i, j} R G B \text {-colored differently } Y_{i, j}\right]_{j=w_{1}, w_{2}, \ldots, w_{n}}^{i=b_{1}, b_{2}, \ldots, b_{n}},
$$

where $b_{1}<b_{2}<\cdots<b_{n}$ are the black nodes and $w_{1}<w_{2}<\cdots<w_{n}$ are the white nodes.

By combining Theorem 1.3.2 with the Desnanot-Jacobi identity, we prove our main result:

Theorem 1.3.3. Let $G=\left(V_{1}, V_{2}, E\right)$ be a finite edge-weighted planar bipartite graph with a set of nodes $\mathbf{N}$. Divide the nodes into three circularly contiguous sets $R, G$, and $B$ such that $|R|,|G|$, and $|B|$ satisfy the triangle inequality and let $\sigma$ be the corresponding tripartite pairing. If $x, w \in V_{1}$ and $y, v \in V_{2}$ then

$$
\begin{aligned}
& \operatorname{sign}_{O E}(\sigma) \operatorname{sign}_{O E}\left(\sigma_{x y w v}^{\prime}\right) Z_{\sigma}^{D D}(G, \mathbf{N}) Z_{\sigma_{x y w v}}^{D D}(G, \mathbf{N}-\{x, y, w, v\}) \\
= & \operatorname{sign}_{O E}\left(\sigma_{x y}^{\prime}\right) \operatorname{sign}_{O E}\left(\sigma_{w v}^{\prime}\right) Z_{\sigma_{x y}}^{D D}(G, \mathbf{N}-\{x, y\}) Z_{\sigma_{w v}}^{D D}(G, \mathbf{N}-\{w, v\}) \\
& -\operatorname{sign}_{O E}\left(\sigma_{x v}^{\prime}\right) \operatorname{sign}_{O E}\left(\sigma_{w y}^{\prime}\right) Z_{\sigma_{x v}}^{D D}(G, \mathbf{N}-\{x, v\}) Z_{\sigma_{w y}}^{D D}(G, \mathbf{N}-\{w, y\}),
\end{aligned}
$$

where for $i, j \in\{x, y, w, v\}, \sigma_{i j}$ is the unique planar pairing on $\mathbf{N}-\{i, j\}$ in which like colors are not paired together, and $\sigma_{i j}^{\prime}$ is the pairing after the the node set $\mathbf{N}-\{i, j\}$ has been relabeled so that the nodes are numbered consecutively.

Theorem 1.0.2 follows as a corollary; the additional assumptions in Theorem 1.0.2 lead to a nice simplification of the signs in Theorem 1.3.3.

As discussed, Theorems 1.3.1 and 1.3.2 generalize the combinatorial results of [KW11a, KW09, KW11b]. The main questions of interest in these bodies of work involve asymptotic and probabilistic properties of the double-dimer model, which were further studied in [Ken14, Dub19, GR19]. In [KP16], Kenyon and Pemantle give a connection between the double-dimer model and cluster algebras. None of these results required taking minors of the matrices from Theorem 1.2.1, so the assumption that the nodes of $G$ are black and odd or white and even was convenient and suitable for their purposes. 


\section{Proof of Theorem 1.3.1}

In this paper, $G$ always denotes a finite edge-weighted bipartite planar graph embedded in the plane with a set of $2 n$ nodes $\mathbf{N}$ on the outer face of $G$ numbered consecutively in counterclockwise order. Kenyon and Wilson [KW11a, KW09] assume that the nodes alternate in color so that the black nodes are odd and the white nodes are even. We allow the nodes to have any coloring, as long as $\mathbf{N}$ has an equal number of black and white nodes.

To prove Theorem 1.3.1, we need to prove analogues of Lemmas $3.1-3.5$ and Theorem 3.6 from Kenyon and Wilson [KW11a] in this more general setting. For ease of exposition, we prove our lemmas in a slightly different order.

2.1. Lemma 3.4 from Kenyon and Wilson. The purpose of this section is to prove an analogue of the following lemma from Kenyon and Wilson [KW11a] for black-white pairings.

Lemma 2.1.1. [KW11a, Lemma 3.4] For odd-even pairings $\rho$,

$$
\operatorname{sign}_{O E}(\rho) \prod_{(i, j) \in \rho}(-1)^{(|i-j|-1) / 2}=(-1)^{\# \text { crosses of } \rho} .
$$

A cross of a pairing $\rho$ is a set of two pairs $(a, c)$ and $(b, d)$ of $\rho$ such that $a<b<c<d$. Recall from Section 1.3 that the sign of an odd-even pairing $\rho=((1, \rho(1)),(3, \rho(3)), \ldots,(2 n-1, \rho(2 n-1)))$ is the parity of the permutation $\left(\begin{array}{llll}\frac{\rho(1)}{2} & \frac{\rho(3)}{2} & \ldots & \frac{\rho(2 n-1)}{2}\end{array}\right)$ written in one-line notation.

For our version of this lemma, we need to define the sign of a black-white pairing $\rho$, which we will denote $\operatorname{sign}_{B W}(\rho)$.

Definition 2.1.2. If $\rho$ is a black-white pairing, then we can write $\rho=\left(\left(b_{1}, w_{1}\right),\left(b_{2}, w_{2}\right), \ldots,\left(b_{n}, w_{n}\right)\right)$, where $b_{1}<b_{2}<\cdots<b_{n}$. Let $r:\left\{w_{1}, \ldots, w_{n}\right\} \rightarrow\{1, \ldots, n\}$ be the map defined by $r(k)=\#\left\{i: w_{i} \leq w_{k}\right\}$. Then the sign of $\rho$, denoted $\operatorname{sign}_{B W}(\rho)$, is the parity of the permutation

$$
\sigma_{\rho}=\left(\begin{array}{llll}
r\left(w_{1}\right) & r\left(w_{2}\right) & \cdots & r\left(w_{n}\right)
\end{array}\right)
$$

written in one-line notation.

When $\rho$ is a pairing that is both black-white and odd-even, these signs agree.

Lemma 2.1.3. If $\rho$ is a black-white pairing that is also odd-even, then $\operatorname{sign}_{O E}(\rho)=\operatorname{sign}_{B W}(\rho)$.

The proof of Lemma 2.1.3 is straightforward, but it is postponed to Section 2.1.3 for clarity of exposition.

In Lemma 2.1.1, the sign of a pair $(i, j)$ of $\rho$ is $(-1)^{(|i-j|-1) / 2}$. If $\rho$ is a black-white pairing that is not odd-even and $(b, w)$ is a pair in $\rho$, it is not necessarily the case that $\frac{|b-w|-1}{2}$ is an integer. Therefore we need a different way to define the sign of a pair.

To motivate this definition, notice that if two nodes of the opposite color $b$ and $w$ have the same parity, it cannot be the case that the nodes between $b$ and $w$ alternate black and white. Therefore we must keep track of the number of consecutive nodes of the same color between $b$ and $w$. Consecutive nodes of the same color appear in pairs. For example, if we have a graph with eight nodes so that nodes $1,3,4$, and 6 are black and nodes $2,5,7,8$ are white, there are two pairs of consecutive nodes of the same color: $(3,4)$ and $(7,8)$. Since we frequently use the term pair when describing pairings of the nodes, we will refer to pairs of consecutive nodes as couples of consecutive nodes instead.

Definition 2.1.4. If $(b, w)$ is a pair in a black-white pairing, let $a_{b, w}$ be the number of couples of consecutive nodes of the same color in the interval $[\min \{b, w\}, \min \{b, w\}+1, \ldots, \max \{b, w\}]$.

We note that a triple of consecutive nodes that are all the same color contributes 2 to $a_{b, w}$.

Remark 2.1.5. If $(b, w)$ is a pair in a black-white pairing, then $\frac{|b-w|+a_{b, w}-1}{2}$ is an integer. 
Proof. Let $\left(n_{1}, n_{1}+1\right),\left(n_{2}, n_{2}+1\right), \ldots,\left(n_{2 k}, n_{2 k}+1\right)$ be a complete list of couples of consecutive nodes of the same color in $\mathbf{N}$ so that $n_{1}<n_{2}<\cdots<n_{2 k}$, where it is possible that $n_{i+1}=n_{i}+1$. Every time we reach a couple of consecutive nodes, the black nodes and white nodes switch parity. That is, if the black nodes in the interval $\left[n_{\ell}+1, n_{\ell}+2, \ldots, n_{\ell+1}\right]$ are odd, then the black nodes in the interval $\left[n_{\ell+1}+1, n_{\ell+1}+2, \ldots, n_{\ell+2}\right]$ are even. (Note that these intervals could be length 1 ). It follows that if $b$ and $w$ are the same parity, then there are an odd number of couples of consecutive nodes in the interval $[\min \{b, w\}, \min \{b, w\}+1, \ldots, \max \{b, w\}]$. So in this case $\frac{|b-w|+a_{b, w}-1}{2}$ is an integer. If $b$ and $w$ are opposite parity, then there are an even number of couples of consecutive nodes in the interval $[\min \{b, w\}, \min \{b, w\}+1, \ldots, \max \{b, w\}]$. So $\frac{|b-w|+a_{b, w}-1}{2}$ is an integer in this case as well.

Definition 2.1.6. If $(b, w)$ is a pair in a black-white pairing, let

$$
\operatorname{sign}(b, w)=(-1)^{\left(|b-w|+a_{b, w}-1\right) / 2} \text {. }
$$

We observe that when the nodes of $G$ alternate black and white, $a_{b, w}=0$ for all pairs $(b, w)$, so this definition of the sign of a pair agrees with Kenyon and Wilson's definition.

Remark 2.1.7. For the remainder of the paper, we use the following notation. We let

- $\left(n_{1}, n_{1}+1\right),\left(n_{2}, n_{2}+1\right), \ldots,\left(n_{2 k}, n_{2 k}+1\right)$ be a complete list of couples of consecutive nodes of the same color so that $n_{1}<\cdots<n_{2 k}$,

- $\left(s_{1}, s_{1}+1\right),\left(s_{2}, s_{2}+1\right), \ldots,\left(s_{k}, s_{k}+1\right)$ be a complete list of couples of consecutive black nodes so that $s_{1}<\cdots<s_{k}$, and

- $\left(u_{1}, u_{1}+1\right),\left(u_{2}, u_{2}+1\right), \ldots,\left(u_{k}, u_{k}+1\right)$ be a complete list of couples of consecutive white nodes so that $u_{1}<\cdots<u_{k}$.

Note that we could have $n_{i+1}=n_{i}+1, s_{i+1}=s_{i}+1$, or $u_{i+1}=u_{i}+1$.

Since we are allowing arbitrary node colorings, many of our results contain a global sign that depends on the order in which the couples of consecutive nodes appear. For example, suppose a node set $\mathbf{N}$ has two couples of consecutive nodes: a couple of consecutive black nodes $(s, s+1)$ and a couple of consecutive white nodes $(u, u+1)$. Then the global sign will be 1 if $u<s$ and -1 if $s<u$. To emphasize that this sign only depends on the relative ordering of the couples of consecutive nodes of the same color, we use the notation $\operatorname{sign}_{\mathrm{c}}(\mathbf{N})$.

Definition 2.1.8. Using the notation from Remark 2.1.7, if node 1 is black, define the map $\varphi:\left\{n_{1}, n_{2}, \ldots, n_{2 k}\right\} \rightarrow\{1,2, \ldots, 2 k\}$ by

$$
\varphi\left(n_{j}\right)=\left\{\begin{array}{ll}
2 i-1 & \text { if } n_{j}=u_{i} \\
2 i & \text { if } n_{j}=s_{i}
\end{array} .\right.
$$

Then the image of $\left\{n_{1}, n_{2}, \ldots, n_{2 k}\right\}$ under the map $\varphi$ can be considered as a permutation in one-line notation:

$$
\sigma_{\mathbf{N}}=\left(\begin{array}{llll}
\varphi\left(n_{1}\right) & \varphi\left(n_{2}\right) \quad \cdots \quad \varphi\left(n_{2 k}\right)
\end{array}\right) .
$$

Define $\operatorname{sign}_{\mathrm{c}}(\mathbf{N})$ to be the sign of this permutation. Note that if $u_{1}<s_{1}<u_{2}<s_{2}<\cdots<u_{k}<s_{k}$ then $\sigma_{\mathbf{N}}=\left(\begin{array}{llll}1 & 2 & \cdots & 2 k\end{array}\right)$, so $\operatorname{sign}_{\mathrm{c}}(\mathbf{N})=1$.

If node 1 is white, define the map $\varphi:\left\{n_{1}, n_{2}, \ldots, n_{2 k}\right\} \rightarrow\{1,2, \ldots, 2 k\}$ by

$$
\varphi\left(n_{j}\right)=\left\{\begin{array}{ll}
2 i-1 & \text { if } n_{j}=s_{i} \\
2 i & \text { if } n_{j}=u_{i}
\end{array} .\right.
$$

As above, the image of $\left\{n_{1}, n_{2}, \ldots, n_{2 k}\right\}$ under the map $\varphi$ can be considered as a permutation in one-line notation and we define $\operatorname{sign}_{\mathrm{c}}(\mathbf{N})$ to be the sign of this permutation. Note that if $s_{1}<u_{1}<s_{2}<u_{2}<\cdots<s_{k}<u_{k}, \operatorname{sign}_{\mathrm{c}}(\mathbf{N})=1$.

In the case where there are no consecutive nodes of the same color, we define $\operatorname{sign}_{c}(\mathbf{N})=1$. 
In Definition 2.1.8, if node 1 is black, it is possible that $s_{k}=2 n$. Similarly, if node 1 is white, it is possible that $u_{k}=2 n$.

Definition 2.1.9. Since the image of $\left\{n_{1}, n_{2}, \ldots, n_{2 k}\right\}$ under the map $\varphi$ can be considered a permutation in one-line notation, we say that a pair $\left(u_{\ell}, s_{m}\right)$ is an inversion with respect to the node coloring of $\mathbf{N}$ if $\left(\varphi\left(u_{\ell}\right), \varphi\left(s_{m}\right)\right)$ is an inversion of $\sigma_{\mathbf{N}}$.

Example 2.1.10. Let $\mathbf{N}$ be a set of nodes where node 1 is black.

- If $\mathbf{N}$ has four couples of consecutive nodes of the same color with $u_{1}<s_{1}<s_{2}<u_{2}$, then $\sigma_{\mathbf{N}}=\left(\begin{array}{llll}1 & 2 & 4 & 3\end{array}\right)$, so $\operatorname{sign}_{\mathrm{c}}(\mathbf{N})=-1$. The pair $\left(s_{2}, u_{2}\right)$ is an inversion with respect to the node coloring.

- If instead $s_{1}<u_{1}<s_{2}<u_{2}$, then $\sigma_{\mathbf{N}}=\left(\begin{array}{llll}2 & 1 & 4 & 3\end{array}\right)$, so $\operatorname{sign}_{\mathrm{c}}(\mathbf{N})=1$. The pairs $\left(s_{1}, u_{1}\right)$ and $\left(s_{2}, u_{2}\right)$ are inversions.

Example 2.1.11. Let $\mathbf{N}$ be a set of nodes where node 1 is white. If $\mathbf{N}$ has six couples of consecutive nodes of the same color with $s_{1}<s_{2}<u_{1}<u_{2}<u_{3}<s_{3}$, then $\sigma_{\mathbf{N}}=\left(\begin{array}{llllll}1 & 3 & 2 & 4 & 6 & 5\end{array}\right)$. The pairs $\left(s_{2}, u_{1}\right)$ and $\left(u_{3}, s_{3}\right)$ are inversions.

Remark 2.1.12. If node 1 is black, $\left(u_{\ell}, s_{m}\right)$ is an inversion with respect to the node coloring when $u_{\ell}<s_{m}$ and $\ell>m$. The pair $\left(s_{m}, u_{\ell}\right)$ is an inversion when $s_{m}<u_{\ell}$ and $m \geq \ell$. If node 1 is white, $\left(u_{\ell}, s_{m}\right)$ is an inversion with respect to the node coloring when $u_{\ell}<s_{m}$ and $\ell \geq m$. The pair $\left(s_{m}, u_{\ell}\right)$ is an inversion when $s_{m}<u_{\ell}$ and $m>\ell$.

We have now established the definitions needed for our version of Kenyon and Wilson's lemma.

Lemma 2.1.13 (analogue of Lemma 3.4 from [KW11a]). If $\rho$ is a black-white pairing,

$$
\operatorname{sign}_{c}(\mathbf{N}) \operatorname{sign}_{B W}(\rho) \prod_{(b, w) \in \rho} \operatorname{sign}(b, w)=(-1)^{\# \text { crosses of } \rho} \text {. }
$$

We remark that in Kenyon and Wilson's case where all black nodes are odd and all white nodes are even, there are no consecutive nodes of the same color, so for all $(b, w) \in \rho, a_{b, w}=0$ and thus $\operatorname{sign}(b, w)=(-1)^{\left(|b-w|+a_{b, w}-1\right) / 2}=(-1)^{(|b-w|-1) / 2}$. If all black nodes are odd and all white nodes are even, a black-white pairing is also an odd-even pairing, and by Lemma 2.1.3, $\operatorname{sign}_{B W}(\rho)=\operatorname{sign}_{O E}(\rho)$. Finally, by Definition 2.1.8, $\operatorname{sign}_{\mathrm{c}}(\mathbf{N})=1$. So in this case, Lemma 2.1.13 agrees exactly with Lemma 2.1.1.

Before proving Lemma 2.1.13, we will prove the following:

Lemma 2.1.14. There exists a planar black-white pairing $\rho$ such that

$$
\operatorname{sign}_{B W}(\rho) \prod_{(b, w) \in \rho} \operatorname{sign}(b, w)=\operatorname{sign}_{c}(\mathbf{N}) .
$$

2.1.1. Proof of Lemma 2.1.14. We will prove Lemma 2.1.14 by induction on $k$, where $\mathbf{N}$ is a set of $2 n$ nodes with $2 k$ couples of consecutive nodes of the same color. The following lemma is the base case $k=1$.

Lemma 2.1.15 (Base case of Lemma 2.1.14). For any node coloring such that there are exactly two couples of consecutive nodes of the same color, there is a planar black-white pairing $\rho$ such that

$$
\operatorname{sign}_{B W}(\rho) \prod_{(b, w) \in \rho} \operatorname{sign}(b, w)=\operatorname{sign}_{c}(\mathbf{N}) .
$$

Proof. Let $\left(n_{1}, n_{1}+1\right),\left(n_{2}, n_{2}+1\right)$ be the list of the couples of consecutive nodes of the same color so that $n_{1}<n_{2}$. There are two cases to consider: Either $n_{1}$ and 1 are opposite colors, or $n_{1}$ and 1 are the same color. 
If $n_{1}$ and 1 are opposite colors, the pairing $\rho=((1,2),(3,4), \ldots,(2 n-1,2 n))$ is black-white. To see this, note that since $n_{1}$ and 1 are opposite colors, $n_{1}$ is even, so the only pairs of adjacent nodes that are both the same color are of the form $(x, x+1)$, where $x$ is even, or $(2 n, 1)$. Since all pairs of $\rho$ are of the form $(i, i+1)$ where $i$ is odd and $i+1$ is even, $\rho$ is a black-white pairing. By the previous definitions we deduce that $\operatorname{sign}_{B W}(\rho)=1$ and $\prod_{(b, w) \in \rho} \operatorname{sign}(b, w)=1$. Since we assumed $n_{1}$ and 1 are opposite colors, either $u_{1}<s_{1}$ (if 1 is black) or $s_{1}<u_{1}$ (if 1 is white) and thus $\operatorname{sign}_{\mathrm{c}}(\mathbf{N})=1$ by Definition 2.1.8. Therefore the claim holds.

If $n_{1}$ and 1 are the same color, the pairing $\rho=((2 n, 1),(2,3), \ldots,(2 n-2,2 n-1))$ is black-white. The reasoning is analogous to the previous case: $n_{1}$ is odd, so the only pairs of adjacent nodes that are both the same color are of the form $(x, x+1)$, where $x$ is odd. In this case, $\operatorname{sign}_{B W}(\rho)=(-1)^{n-1}$ and $\prod_{(b, w) \in \rho} \operatorname{sign}(b, w)=(-1)^{\left(2 n-1+a_{2 n, 1}-1\right) / 2}=(-1)^{n-1}(-1)^{a_{2 n, 1} / 2}$, so

$$
\operatorname{sign}_{B W}(\rho) \prod_{(b, w) \in \rho} \operatorname{sign}(b, w)=(-1)^{a_{2 n, 1} / 2}=(-1)^{2 / 2}=-1 .
$$

Similar to the previous case, since we assumed $n_{1}$ and 1 are the same color, $\operatorname{sign}_{\mathrm{c}}(\mathbf{N})=-1$, and thus the claim holds.

Definition 2.1.16. Suppose $\rho$ is a black-white pairing. Then recall that we can write $\rho=\left(\left(b_{1}, w_{1}\right),\left(b_{2}, w_{2}\right), \ldots,\left(b_{n}, w_{n}\right)\right)$, where $b_{1}<b_{2}<\cdots<b_{n}$. We say that $\left(w_{i}, w_{j}\right)$ is an inversion of $\rho$ if $i<j$ and $w_{i}>w_{j}$. Note that $\left(w_{i}, w_{j}\right)$ is an inversion of $\rho$ if and only if $\left(r\left(w_{i}\right), r\left(w_{j}\right)\right)$ is an inversion of $\sigma_{\rho}$ (see Definition 2.1.2).

Definition 2.1.17. Similarly, if $\pi$ is an odd-even pairing, then we can write $\pi=((1, \pi(1)),(3, \pi(3)), \ldots,(2 n-1, \pi(2 n-1)))$ and we will say $(\pi(i), \pi(j))$ is an inversion of $\pi$ if $i<j$ and $\pi(i)>\pi(j)$. This is equivalent to defining an inversion of $\pi$ to be an inversion of the permutation $\left(\begin{array}{llll}\frac{\pi(1)}{2} & \frac{\pi(3)}{2} & \ldots & \frac{\pi(2 n-1)}{2}\end{array}\right)$.
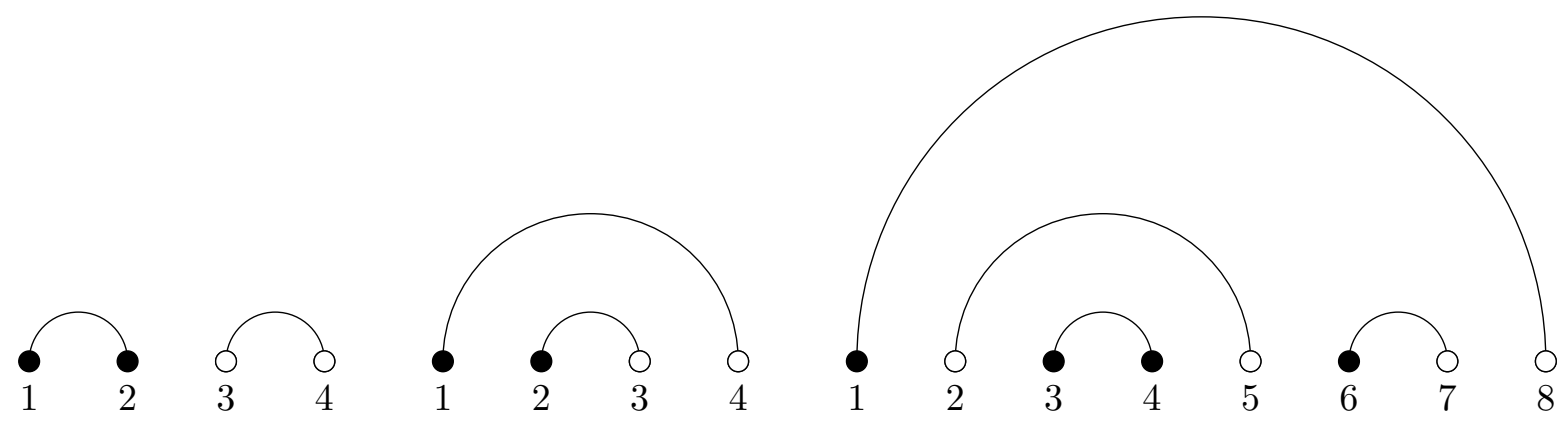

Figure 4. An inversion of a planar pairing $\pi$ corresponds to a nesting. Left: the pairing $((1,2),(3,4))$ has no inversions and its diagram has no nestings. Center: The pairing $((1,4),(3,2))$ has one inversion and its diagram has one nesting. Right: The pairing $((1,8),(3,4),(5,2),(7,6))$ has four inversions and four nestings: $\{(1,8),(3,4)\},\{(1,8),(5,2)\},\{(1,8),(7,6)\}$, and $\{(3,4),(5,2)\}$.

Remark 2.1.18. In the special case where an odd-even pairing $\pi$ is also planar, we remark that an inversion of $\pi$ corresponds to a nesting in the diagram constructed by placing the nodes in order on a line and linking pairs in the upper half-plane. More precisely, two $\operatorname{arcs}\left(a_{1}, b_{1}\right),\left(a_{2}, b_{2}\right)$ are said to be nesting if $a_{1}<a_{2}<b_{2}<b_{1}$ (see Figure 4). This correspondence between inversions and nestings follows immediately from the four node case, where the only planar pairings are $((1,2),(3,4))$ and $((1,4),(3,2))$. 
Proof of Lemma 2.1.14. The proof of the lemma is technical, so we first identify a few easy cases.

Easy case 1. If

- node 1 is black and $u_{1}<s_{1}<u_{2}<s_{2}<\cdots<u_{k}<s_{k}$, or

- node 1 is white and $s_{1}<u_{1}<s_{2}<u_{2}<\cdots<s_{k}<u_{k}$,

then as in the proof of the first case of Lemma 2.1.15, the pairing $((1,2),(3,4), \ldots,(2 n-1,2 n))$ is a planar black-white pairing with $\operatorname{sign}_{B W}(\rho)=1, \prod_{(b, w) \in \rho} \operatorname{sign}(b, w)=1$, and $\operatorname{sign}_{\mathrm{c}}(\mathbf{N})=1$. Thus the claim holds.

\section{Easy case 2. If}

- node 1 is black and $s_{1}<u_{1}<s_{2}<u_{2}<\cdots<s_{k}<u_{k}$, or

- node 1 is white and $u_{1}<s_{1}<u_{2}<s_{2}<\cdots<u_{k}<s_{k}$,

then the pairing $\rho=((2 n, 1),(2,3), \ldots,(2 n-2,2 n-1))$ is black-white (as in the second case of Lemma 2.1.15). In this case,

- $\operatorname{sign}_{B W}(\rho)=(-1)^{n-1}$, and

- $\prod_{(b, w) \in \rho} \operatorname{sign}(b, w)=(-1)^{\left(2 n-1+a_{2 n, 1}-1\right) / 2}=(-1)^{n-1}(-1)^{a_{2 n, 1} / 2}=(-1)^{n-1}(-1)^{k}$,

so $\operatorname{sign}_{B W}(\rho) \prod_{(b, w) \in \rho} \operatorname{sign}(b, w)=(-1)^{k}=\operatorname{sign}_{\mathrm{c}}(\mathbf{N})$.

General case. For the general case, we proceed by induction on the number of couples of consecutive nodes of the same color. The base case is when there are two couples of consecutive nodes of the same color, which is Lemma 2.1.15. Assume the claim holds when we have a set of nodes that has $2(k-1)$ couples of consecutive nodes of the same color and let $\mathbf{N}$ be a set of nodes with $2 k$ couples of consecutive nodes of the same color.

Using the notation from Remark 2.1.7, let $h$ be the smallest integer so that $n_{h-1}$ and $n_{h}$ are different colors. Then $\rho_{1}=\left(\left(n_{h-1}+1, n_{h-1}+\right.\right.$ $\left.2), \ldots,\left(n_{h}-1, n_{h}\right)\right)$ is a black-white pairing that contains at least one pair.

Throughout this proof, we will illustrate the main ideas with the example where $\mathbf{N}$ is a set of 12 nodes colored so that nodes $1,3,4,5,7$, and 10 are black, as shown to the right. In this example, the couples of consecutive nodes of the same color are $(3,4),(4,5),(8,9)$, and $(11,12)$. Since $n_{1}=3$ and $n_{2}=4$ are black and $n_{3}=8$ is white, $h=3$. So the pairing $\rho_{1}$ is $((5,6),(7,8))$.

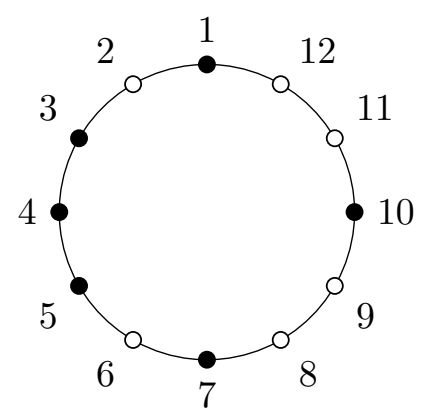

Consider $\mathbf{N}^{\prime}=\left\{1, \ldots,|\mathbf{N}|-\left(n_{h}-n_{h-1}\right)\right\}$. Define $\psi: \mathbf{N}-\left\{n_{h-1}+1, \ldots, n_{h}\right\} \rightarrow \mathbf{N}^{\prime}$ by

$$
\psi(\ell)= \begin{cases}\ell & \text { if } \ell \leq n_{h-1} \\ \ell-\left(n_{h}-n_{h-1}\right) & \text { if } \ell>n_{h}\end{cases}
$$

That is, $\psi$ defines a relabeling of the nodes of $\mathbf{N}-\left\{n_{h-1}+1, \ldots, n_{h}\right\}$ so that node 1 is labeled $1, \ldots$, node $n_{h-1}$ is labeled $n_{h-1}$, node $n_{h}+1$ is labeled $n_{h-1}+1, \ldots$, node $2 n$ is labeled $2 n-\left(n_{h}-n_{h-1}\right)$. Since $\mathbf{N}^{\prime}$ has $2 k-2$ couples of consecutive nodes of the same color, by the induction hypothesis there is a black-white planar pairing $\rho_{2}$ of the nodes of $\mathbf{N}^{\prime}$ such that

$$
\operatorname{sign}_{B W}\left(\rho_{2}\right) \prod_{(b, w) \in \rho_{2}} \operatorname{sign}(b, w)=\operatorname{sign}_{\mathbf{c}}\left(\mathbf{N}^{\prime}\right) .
$$

Let $\psi^{-1}\left(\rho_{2}\right)$ denote the pairing that results from applying $\psi^{-1}$ to each node in $\rho_{2}$. That is, $\psi^{-1}\left(\rho_{2}\right)$ is the pairing obtained by returning the nodes of $\rho_{2}$ to their original labels in $\mathbf{N}$. Let $\rho=\rho_{1} \cup \psi^{-1}\left(\rho_{2}\right)$. Observe that $\rho$ is a planar black-white pairing of $\mathbf{N}$. 

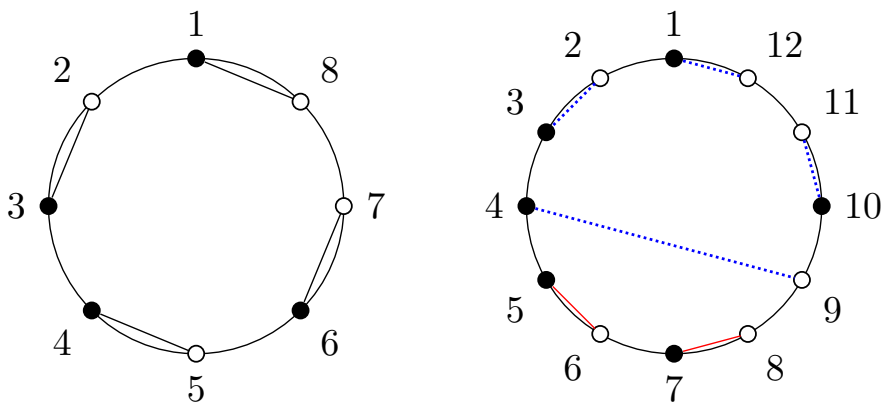

Figure 5. Left: The pairing $\rho_{2}$ of $\mathbf{N}^{\prime}$ guaranteed by the induction hypothesis. Right: The pairing $\rho$ of $\mathbf{N}$. The pairing $\rho_{1}$ is shown with solid red lines and the pairing $\psi^{-1}\left(\rho_{2}\right)$ is shown with dotted blue lines.

In our example, the map $\psi$ defines a relabeling of $\mathbf{N}-\{5,6,7,8\}$ so that node 9 is labeled $5, \ldots$, node 12 is labeled 8 . The node set $\mathbf{N}^{\prime}$ has two couples of consecutive pairs of the same color. By Lemma 2.1 .15 , the pairing $\rho_{2}$ is $((1,8),(3,2),(5,4),(7,6))$, so the pairing $\psi^{-1}\left(\rho_{2}\right)$ is $((1,12),(3,2),(9,4),(11,10))$ and thus $\rho=((1,12),(3,2),(5,6),(7,8),(9,4),(11,10))$, as shown in Figure 5.

We will next

(1) Compare $\prod_{(b, w) \in \rho_{2}} \operatorname{sign}(b, w)$ to $\prod_{(b, w) \in \rho} \operatorname{sign}(b, w)$,

(2) Compare $\operatorname{sign}_{B W}\left(\rho_{2}\right)$ to $\operatorname{sign}_{B W}(\rho)$, and

(3) Compare $\operatorname{sign}_{\mathrm{c}}\left(\mathbf{N}^{\prime}\right)$ to $\operatorname{sign}_{\mathrm{c}}(\mathbf{N})$.

(1) Comparing $\prod_{(b, w) \in \rho_{2}} \operatorname{sign}(b, w)$ to $\prod_{(b, w) \in \rho} \operatorname{sign}(b, w)$. If $(b, w)$ is a pair in $\rho$ that is a pair of $\rho_{1}$, then $\operatorname{sign}(b, w)=1$. If $(b, w)$ is a pair in $\rho$ that is a pair of $\psi^{-1}\left(\rho_{2}\right)$, then consider $(\psi(b), \psi(w))$ (the corresponding pair of $\rho_{2}$ ). If $b, w \leq n_{h-1}$ or $b, w \geq n_{h}+1$, then $\operatorname{sign}(b, w)=\operatorname{sign}(\psi(b), \psi(w)$ ) because $a_{b, w}=a_{\psi(b), \psi(w)}$. If $w \leq n_{h-1}$ and $b \geq n_{h}+1$ then

$$
\begin{aligned}
\operatorname{sign}(\psi(b), \psi(w))=(-1)^{\left(\psi(b)-\psi(w)+a_{\psi(b), \psi(w)}-1\right) / 2} & =(-1)^{\left(b-\left(n_{h}-n_{h-1}\right)-w+a_{b, w}-2-1\right) / 2} \\
& =(-1)^{\left.\left(-\left(n_{h}-n_{h-1}\right)-2\right)\right) / 2} \operatorname{sign}(b, w) \\
& =(-1)^{\left(n_{h}-n_{h-1}+2\right) / 2} \operatorname{sign}(b, w) .
\end{aligned}
$$

so

$$
\prod_{(b, w) \in \rho_{2}} \operatorname{sign}(b, w)=\prod_{\substack{(b, w) \in \rho: \\ \min (b, w) \leq n_{h-1} \text { and } \\ \max (b, w) \geq n_{h}+1}}(-1)^{\left(n_{h}-n_{h-1}+2\right) / 2} \prod_{(b, w) \in \rho} \operatorname{sign}(b, w)
$$

In the example, there are two pairs $(b, w)$ with $\min (b, w) \leq n_{2}$ and $\max (b, w) \geq n_{3}+1$ : the pairs $(1,12)$ and $(4,9)$.

(2) Comparing $\operatorname{sign}_{B W}\left(\rho_{2}\right)$ to $\operatorname{sign}_{B W}(\rho)$. Comparing $\operatorname{sign}_{B W}\left(\rho_{2}\right)$ to $\operatorname{sign}_{B W}(\rho)$ requires comparing the number of inversions of $\rho$ to the number of inversions of $\rho_{2}$ (see Definition 2.1.16). Since $\rho_{1}$ contains only pairs of the form $(i, i+1), \rho_{1}$ contains no inversions. Since the pairings under consideration are planar we can use the fact that inversions of a planar pairing correspond to nestings in the corresponding diagram (see Remark 2.1.18). Since there are $\frac{n_{h}-n_{h-1}}{2}$ pairs in $\rho_{1}, \rho$ has $\frac{n_{h}-n_{h-1}}{2}$ additional inversions compared to $\rho_{2}$ for each pair $(b, w)$ such that $\min (b, w) \leq n_{h-1}$ 
and $\max (b, w) \geq n_{h}+1$. So,

$$
\operatorname{sign}_{B W}\left(\rho_{2}\right)=\operatorname{sign}_{B W}(\rho) \prod_{\substack{(b, w) \in \rho: \\ \min (b, w) \leq n_{h-1} \text { and } \\ \max (b, w) \geq n_{h}+1}}(-1)^{\left(n_{h}-n_{h-1}\right) / 2}
$$

In the example, since there are two pairs $(b, w)$ with $\min \{b, w\} \leq n_{2}$ and $\max \{b, w\} \geq n_{3}+1$ and the pairing $\rho_{1}$ consists of two pairs, there are four more inversions in $\rho$ than in $\rho_{2}$.

(3) Comparing $\operatorname{sign}_{\mathrm{c}}\left(\mathbf{N}^{\prime}\right)$ to $\operatorname{sign}_{\mathrm{c}}(\mathbf{N})$. We will show that

$$
\operatorname{sign}_{\mathrm{c}}\left(\mathbf{N}^{\prime}\right)=(-1)^{h-1} \operatorname{sign}_{\mathrm{c}}(\mathbf{N})
$$

by comparing the number of inversions with respect to the node coloring of $\mathbf{N}$ to the number of inversions in with respect to the node coloring of $\mathbf{N}^{\prime}$ (see Definition 2.1.9). In particular, we show that there are $(h-2)+(h-1)$ inversions in $\mathbf{N}$ that do not have a counterpart in $\mathbf{N}^{\prime}$ and that there are $h-2$ inversions in $\mathbf{N}^{\prime}$ that do not have a counterpart in $\mathbf{N}$.

Recall the notation from Remark 2.1.7: $s_{i}$ is the first in a couple of consecutive black nodes in $\mathbf{N}$ and $u_{i}$ is the first in a couple of consecutive white nodes in $\mathbf{N}$. Define $s_{i}^{\prime}$ and $u_{i}^{\prime}$ analogously for $\mathbf{N}^{\prime}$.

First assume node 1 is black and that we have

$$
s_{1}<\cdots<s_{h-1}<u_{1}<\cdots .
$$

Inversions with respect to the node coloring of $\mathbf{N}$. By Remark 2.1.12, there are two types of inversions with respect to the node coloring of $\mathbf{N}$.

(1) Nodes $x$ and $y$ in $\mathbf{N}$ such that $x<y, x=s_{a}, y=u_{b}$, and $a \geq b$.

(2) Nodes $x$ and $y$ in $\mathbf{N}$ such that $x<y, x=u_{a}, y=s_{b}$, and $a>b$.

Considering the first type of inversion, there are several cases:

(a) If $a \leq h-2$ and $b>1$, then $\psi(x)=s_{a}^{\prime}$ and $\psi(y)=u_{b-1}^{\prime}$. Since $a \geq b, a \geq b-1$, so in this case there is a corresponding inversion in $\mathbf{N}^{\prime}$.

(b) If $a \leq h-2$ and $b=1$, then $y \notin \mathbf{N}-\left\{n_{h-1}+1, \ldots, n_{h}\right\}$, so in this case there is not a corresponding inversion in $\mathbf{N}^{\prime}$.

(c) If $a=h-1$ and $b \leq h-1$, then $x \notin \mathbf{N}-\left\{n_{h-1}+1, \ldots, n_{h}\right\}$, so in this case there is not a corresponding inversion in $\mathbf{N}^{\prime}$.

(d) If $a>h-1$ and $b \leq a$, then $b>1$ (since $u_{1}<s_{a}$ ). In this case, $\psi(x)=s_{a-1}^{\prime}$ and $\psi(y)=u_{b-1}^{\prime}$, so there is a corresponding inversion in $\mathbf{N}^{\prime}$.

Note that (b) gives $h-2$ inversions in $\mathbf{N}$ that are not in $\mathbf{N}^{\prime}$ and (c) gives $h-1$ inversions in $\mathbf{N}$ that are not in $\mathbf{N}^{\prime}$.

Considering the second type of inversion, since $s_{h-1}<u_{1}$ we must have $a>h$. In this case, $\psi(x)=u_{a-1}^{\prime}$ and $\psi(y)=s_{b-1}^{\prime}$, so there is a corresponding inversion in $\mathbf{N}^{\prime}$.

In the example, the pairs $\left(s_{1}, u_{1}\right),\left(s_{2}, u_{1}\right)$, and $\left(s_{2}, u_{2}\right)$ are inversions with respect to the node coloring of $\mathbf{N}$. Since $h=3$, the inversion $\left(s_{1}, u_{1}\right)$ is in case (b) of the first type and the inversions $\left(s_{2}, u_{1}\right)$ and $\left(s_{2}, u_{2}\right)$ are in case (c) of the first type. So in this example, all of the inversions with respect to the node coloring of $\mathbf{N}$ do not have corresponding inversions in $\mathbf{N}^{\prime}$.

Inversions with respect to the node coloring of $\mathbf{N}^{\prime}$. Similarly, there are two types of inversions in $\mathbf{N}^{\prime}$.

(1) Nodes $w$ and $z$ in $\mathbf{N}^{\prime}$ such that $w<z, w=s_{a}^{\prime}, z=u_{b}^{\prime}$, and $a \geq b$.

(2) Nodes $w$ and $z$ in $\mathbf{N}^{\prime}$ such that $w<z, w=u_{a}^{\prime}, z=s_{b}^{\prime}$, and $a>b$.

Considering the first type of inversion, there are two cases:

(a) If $a \leq h-2$, then $\psi^{-1}(w)=s_{a}$ and $\psi^{-1}(z)=u_{b+1}$.

(i) If $a \geq b+1$ then there is a corresponding inversion in $\mathbf{N}$. 
(ii) If $a=b$ there is not a corresponding inversion in $\mathbf{N}$.

(b) If $a \geq h-1$, then $\psi^{-1}(w)=s_{a+1}$ and $\psi^{-1}(z)=u_{b+1}$, so there is a corresponding inversion in $\mathbf{N}$.

We see that case (a)(ii) gives $h-2$ inversions in $\mathbf{N}^{\prime}$ that are not in $\mathbf{N}$.

Considering the second type of inversion, since $s_{h-2}^{\prime}<u_{1}^{\prime}$ the only possibility is that $a>h-1$. In this case, $\psi^{-1}(w)=u_{a+1}$ and $\psi^{-1}(z)=s_{b+1}$, so there is a corresponding inversion in $\mathbf{N}$.

In the example, the only inversion with respect to the node coloring of $\mathbf{N}^{\prime}$ is $\left(s_{1}^{\prime}, u_{1}^{\prime}\right)$, which is an example of case (a)(ii), so there is not a corresponding inversion in $\mathbf{N}$.

We conclude that in the case where node 1 is black and we have $s_{1}<\cdots<s_{h-1}<u_{1}<\cdots$, the equation $\operatorname{sign}_{\mathrm{c}}\left(\mathbf{N}^{\prime}\right)=(-1)^{h-1} \operatorname{sign}_{\mathrm{c}}(\mathbf{N})$ holds.

Combining this with

- $\operatorname{sign}_{B W}\left(\rho_{2}\right) \prod_{(b, w) \in \rho_{2}} \operatorname{sign}(b, w)=\operatorname{sign}_{\mathrm{c}}\left(\mathbf{N}^{\prime}\right)$,

$$
\begin{gathered}
\text { - } \prod_{(b, w) \in \rho_{2}} \operatorname{sign}(b, w)=\prod_{\substack{(b, w) \in \rho: \\
\min (b, w) \leq n_{h-1} \text { and } \\
\max (b, w) \geq n_{h}+1}}(-1)^{\left(n_{h}-n_{h-1}+2\right) / 2} \prod_{(b, w) \in \rho} \operatorname{sign}(b, w) \text {, and } \\
\text { - } \operatorname{sign}_{B W}\left(\rho_{2}\right)=\operatorname{sign}_{B W}(\rho) \prod_{\substack{(b, w) \in \rho: \\
\min (b, w) \leq n_{h-1} \\
\max (b, w) \geq n_{h}+1}}^{\text {and }}(-1)^{\left(n_{h}-n_{h-1}\right) / 2},
\end{gathered}
$$

we have

$$
\operatorname{sign}_{B W}(\rho) \prod_{(b, w) \in \rho} \operatorname{sign}(b, w)=\operatorname{sign}_{\mathrm{c}}(\mathbf{N}) \cdot(-1)^{h-1} \cdot \prod_{\substack{(b, w) \in \rho: \\ \min (b, w) \leq n_{h-1} \text { and } \\ \max (b, w) \geq n_{h}+1}}(-1) .
$$

So it remains to observe that the number of pairs $(b, w) \in \rho$ such that $\min (b, w) \leq s_{h-1}$ and $\max (b, w) \geq u_{1}+1$ has the same parity as $h-1$. There are exactly $h-1$ more black nodes than white nodes in the interval $\left[1, \ldots, s_{h-1}\right]$ because there are $h-1$ black nodes that are not followed by a white node in this interval. So there are $h-1$ black nodes that must all be paired with a white node with label $\geq u_{1}+1$. Therefore there are at least $h-1$ pairs $(b, w) \in \rho$ such that $\min (b, w) \leq s_{h-1}$ and $\max (b, w) \geq u_{1}+1$. There may be more than $h-1$ such pairs, but there must be $h-1+2 m$ pairs for some $m \geq 0$.

There are three other cases: when node 1 is white and we have $s_{1}<\cdots<s_{h-1}<u_{1}<\cdots$, when node 1 is black and we have $u_{1}<\cdots<u_{h-1}<s_{1}<\cdots$, and when node 1 is white and we have $u_{1}<\cdots<u_{h-1}<s_{1}<\cdots$. These are omitted because the analyses are nearly identical to the case we just considered.

2.1.2. Proof of Lemma 2.1.13. Recall that we want to show that if $\rho$ is a black-white pairing on a graph $G$ with node set $\mathbf{N}$,

$$
\operatorname{sign}_{\mathbf{c}}(\mathbf{N}) \operatorname{sign}_{B W}(\rho) \prod_{(b, w) \in \rho} \operatorname{sign}(b, w)=(-1)^{\# \text { crosses of } \rho} .
$$

By Lemma 2.1.14 there is a black-white planar pairing $\rho$ such that

$$
\operatorname{sign}_{B W}(\rho) \prod_{(b, w) \in \rho} \operatorname{sign}(b, w)=\operatorname{sign}_{\mathrm{c}}(\mathbf{N}) .
$$

Since $\rho$ is planar, $(-1)^{\# \text { crosses of } \rho}=1$, so equation (2.1.2) holds. 
To prove equation (2.1.2) holds for all black-white pairings we consider ways we can modify black-white pairings to obtain new black-white pairings and determine how these modifications affect equation (2.1.2).

Definition 2.1.19. Let $\sigma$ be a (not necessarily black-white) pairing on $\{1, \ldots, 2 n\}$, such that $x$ is not paired with $y$. When we swap the locations of $x$ and $y$ in $\sigma$ we create a new pairing $\sigma^{\prime}$ that is identical to $\sigma$ except that it contains the pairs $(x, \sigma(y))$ and $(y, \sigma(x))$ rather than $(x, \sigma(x))$ and $(y, \sigma(y))$.

Example 2.1.20. Suppose $\sigma$ is the pairing $((1,3),(2,4),(5,6))$. If we swap the locations of 3 and 4 in $\sigma$ we obtain the pairing $\sigma^{\prime}=((1,4),(2,3),(5,6))$.

Remark 2.1.21. If $\rho$ is a black-white pairing and $\rho^{\prime}$ is obtained from $\rho$ by swapping the locations of two nodes of the same color, $\operatorname{sign}_{B W}\left(\rho^{\prime}\right)=-\operatorname{sign}_{B W}(\rho)$.

Now we observe that we can obtain any black-white pairing on $\mathbf{N}$ from a given black-white pairing $\rho$ using the following types of swaps:

(1) Swapping the locations of $u$ and $u+1$ in $\rho$, where $(u, u+1)$ is a couple of consecutive white nodes.

(2) Swapping the locations of $x$ and $y$ in $\rho$, where $x<y$ are white nodes and all $\ell$ nodes appearing between $x$ and $y$ are black, where $\ell \geq 1$.

To see that these swaps are sufficient, let $w_{1}, \ldots, w_{n}$ be the white nodes in increasing order. The swaps described are the adjacent transpositions $\left(w_{1}, w_{2}\right),\left(w_{2}, w_{3}\right), \ldots,\left(w_{n-1}, w_{n}\right)$.

We will show that equation (2.1.2) holds after applying each type of swap. This requires a few additional lemmas. Note that the proofs of Lemmas 2.1.22 through 2.1.24 follow immediately from Definition 2.1.6.

Lemma 2.1.22. Let $b$ be a black node and let $(u, u+1)$ be a couple of consecutive white nodes. Then $\operatorname{sign}(b, u)=-\operatorname{sign}(b, u+1)$.

Proof. If $b<u$, then $a_{b, u+1}=a_{b, u}+1$. So

$$
\operatorname{sign}(b, u)=(-1)^{\left(u-b+a_{b, u}-1\right) / 2}=-(-1)^{\left(u+1-b+a_{b, u+1}-1\right) / 2}=-\operatorname{sign}(b, u+1) .
$$

If $b>u+1$, then $a_{b, u+1}=a_{b, u}-1$. So

$$
\operatorname{sign}(b, u)=(-1)^{\left(b-u+a_{b, u}-1\right) / 2}=-(-1)^{\left(b-(u+1)+a_{b, u+1}-1\right) / 2}=-\operatorname{sign}(b, u+1) .
$$

Lemma 2.1.23. Assume the nodes $x$ and $y$ with $x<y$ are white and all $\ell$ nodes between $x$ and $y$ are black, where $\ell \geq 1$. If $b$ is a black node not in the interval $[x+1, \ldots, y-1]$, then $\operatorname{sign}(b, x)=(-1)^{\ell} \operatorname{sign}(b, y)$.

Proof. If $b<x$, then $a_{b, y}=a_{b, x}+\ell-1$. Then since $y=x+\ell+1$,

$$
\begin{aligned}
\operatorname{sign}(b, x)=(-1)^{\left(x-b+a_{b, x}-1\right) / 2}=(-1)^{\left(y-(\ell+1)-b+a_{b, y}-\ell+1-1\right) / 2} & =(-1)^{\ell}(-1)^{\left(y-b+a_{b, y}-1\right) / 2} \\
& =(-1)^{\ell} \operatorname{sign}(b, y) .
\end{aligned}
$$

If $b>y$, then $a_{b, y}=a_{b, x}-(\ell-1)$. Then

$$
\begin{aligned}
\operatorname{sign}(b, x)=(-1)^{\left(b-x+a_{b, x}-1\right) / 2}=(-1)^{\left(b-(y-(\ell+1))+a_{b, y}+(\ell-1)-1\right) / 2} & =(-1)^{\ell}(-1)^{\left(b-y+a_{b, y}-1\right) / 2} \\
& =(-1)^{\ell} \operatorname{sign}(b, y) .
\end{aligned}
$$

Lemma 2.1.24. Assume the nodes $x$ and $y$ with $x<y$ are white and all $\ell$ nodes between $x$ and $y$ are black, where $\ell \geq 1$. If $b$ is a black node in the interval $[x+1, \ldots, y-1]$, so $b=x+j$ for some $j \leq \ell$, then $\operatorname{sign}(b, x)=(-1)^{\ell-1} \operatorname{sign}(b, y)$. 
Proof. Since $b=x+j$ and $a_{b, x}=j-1$, we see that

$$
\operatorname{sign}(b, x)=(-1)^{\left(b-x+a_{b, x}-1\right) / 2}=(-1)^{(j+j-1-1) / 2}=(-1)^{j-1} .
$$

Using the fact that $y-b=\ell+1-j$ and $a_{b, y}=\ell-j$, we have

$$
\operatorname{sign}(b, y)=(-1)^{\left(y-b+a_{b, y}-1\right) / 2}=(-1)^{(\ell+1-j+\ell-j-1) / 2}=(-1)^{\ell-j} .
$$

So $\operatorname{sign}(b, x)=(-1)^{\ell-1} \operatorname{sign}(b, y)$.

Remark 2.1.25. The symmetric group $S_{2 n}$ acts on the set of pairings on $\{1, \ldots, 2 n\}$ : the transposition $(i, i+1)$ acts on a pairing $\rho$ by swapping the locations of $i$ and $i+1$. If $i$ is paired with $i+1$, acting with $(i, i+1)$ leaves the pairing fixed; otherwise, $(i, i+1)$ acts nontrivially and changes the parity of the number of crossings.

Let $\rho$ be a (not necessarily black-white) pairing on $\{1, \ldots, 2 n\}$. Let $x$ and $y$ be two nodes such that $x<y$. Assume no node in the interval $[x, y]$ is paired with any other node in this interval. Then

$$
(x, y) \rho=(x, x+1) \cdots(y-1, y) \cdots(x+1, x+2)(x, x+1) \rho
$$

where each transposition of the form $(i, i+1)$ acts nontrivially.

Lemma 2.1.26. Let $\rho$ be a (not necessarily black-white) pairing on $\{1, \ldots, 2 n\}$. Let $x$ and $y$ be two nodes such that $x<y$ and $x$ is not paired with $y$. Assume that no node in the interval $[x+1, \ldots, y-1]$ is paired with any other node in this interval. Then when the locations of $x$ and $y$ in $\rho$ are swapped,

(1) if $x$ and $y$ were both paired with nodes in the interval $[x+1, \ldots, y-1]$, the number of crossings of $\rho$ changes parity,

(2) if exactly one of $x$ and $y$ was paired with a node in the interval $[x+1, \ldots, y-1]$, then the number of crossings of $\rho$ does not change parity, and

(3) if neither $x$ nor $y$ was paired with a node in the interval $[x+1, \ldots, y-1]$ then the number of crossings of $\rho$ changes parity.

Proof. Let $\rho$ be a pairing on $\{1, \ldots, 2 n\}$ and consider $(x, y) \rho$. There are several cases. The strategy is to factor $(x, y)$ into adjacent transpositions and determine which transpositions act nontrivially.

Case 1. If the nodes $\rho(x)$ and $\rho(y)$ are both in the interval $[x+1, \ldots, y-1]$, then $(x, y) \rho=$ $(\rho(x), \rho(y)) \rho$. Let $a=\min (\rho(x), \rho(y))$ and let $b=\max (\rho(x), \rho(y))$. Then

$$
(\rho(x), \rho(y)) \rho=(a, a+1) \cdots(b-1, b) \cdots(a+1, a+2)(a, a+1) \rho
$$

We have written $(\rho(x), \rho(y))$ as a product of an odd number of transpositions of the form $(i, i+1)$. Since no node in the interval $[a, \ldots, b]$ is paired with any other node in this interval, all these transpositions act nontrivially by Remark 2.1.25. Thus the parity of the number of crossings changes.

Case 2. If exactly one of the nodes $\rho(x)$ or $\rho(y)$ is in the interval $[x+1, \ldots, y-1]$, then

$$
(x, y) \rho=(x, x+1) \cdots(y-1, y) \cdots(x+1, x+2)(x, x+1) \rho
$$

and exactly one of these transpositions acts trivially. For if $x$ is paired with $x+k$, then after applying the transposition $(x, x+1)$ to $\rho, x+1$ and $x+k$ are paired. Similarly, after applying the transposition $(x+1, x+2)$ to $(x, x+1) \rho, x+2$ and $x+k$ are paired. It follows that the transposition $(x+k-1, x+k)$ acts trivially because when we reach this transposition, $x+k-1$ and $x+k$ are paired. Then, the transposition $(x+k-1, x+k-2)$ acts nontrivially and similarly we see that the remaining transpositions act nontrivially. Since an even number of transpositions of the form $(i, i+1)$ act nontrivially, the parity of the number of crossings does not change. 
Case 3. If neither of the nodes $\rho(x)$ and $\rho(y)$ are in the interval $[x+1, \ldots, y-1]$, then

$$
(x, y) \rho=(x, x+1) \cdots(y-1, y) \cdots(x+1, x+2)(x, x+1) \rho
$$

so we have written $(\rho(x), \rho(y))$ as a product of an odd number of transpositions of the form $(i, i+1)$. Since no node in the interval $[x, y]$ is paired with any other node in this interval, all of these transpositions act nontrivially by Remark 2.1.25. Thus the parity of the number of crossings changes.

Now that we have established Lemmas 2.1.22 through 2.1.26 we can show that equation (2.1.2) holds after applying both types of swaps to $\rho$. By Remark 2.1.21, each swap changes $\operatorname{sign}_{B W}(\rho)$.

(1) Swapping the locations of $u$ and $u+1$.

Let $b_{1}$ be the node paired with $u$ and let $b_{2}$ be the node paired with $u+1$. By Lemma 2.1.22, $\operatorname{sign}\left(b_{1}, u\right)=-\operatorname{sign}\left(b_{1}, u+1\right)$ and $\operatorname{sign}\left(b_{2}, u+1\right)=-\operatorname{sign}\left(b_{2}, u\right)$. So when we swap the locations of

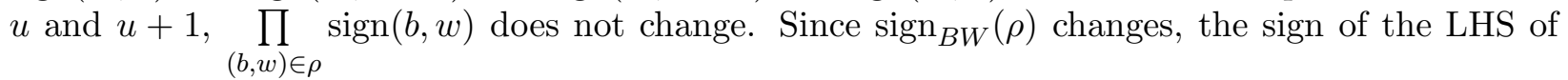

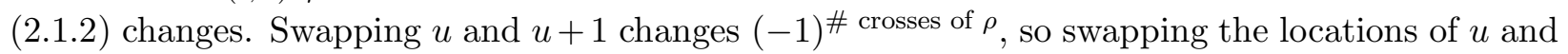
$u+1$ does not affect equation (2.1.2).

(2) Swapping the locations of $x$ and $y$, where $x<y$ are white nodes and all $\ell$ nodes between $x$ and $y$ are black.

Case 1. If $x$ and $y$ are both paired with black nodes in the interval $[x+1, x+2, \ldots, y-1]$, then

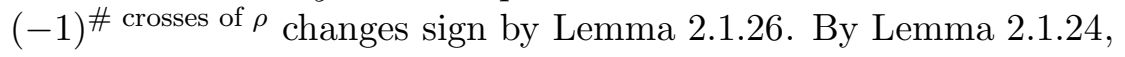

$$
\operatorname{sign}(\rho(x), x) \operatorname{sign}(\rho(y), y)=\left((-1)^{\ell-1}\right)^{2} \operatorname{sign}(\rho(x), y) \operatorname{sign}(\rho(y), x)
$$

so $\prod_{(b, w) \in \rho} \operatorname{sign}(b, w)$ does not change. $\operatorname{Since}_{\operatorname{sign}} \operatorname{si}_{W}(\rho)$ changes, the sign of the LHS of (2.1.2) changes.

Case 2. If exactly one of $x$ and $y$ is paired with a black node in the interval $[x+1, x+2, \ldots, y-1]$,

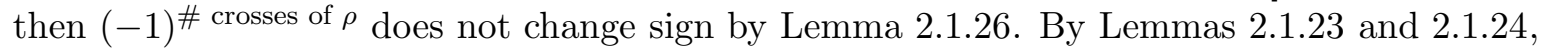

$$
\operatorname{sign}(\rho(x), x) \operatorname{sign}(\rho(y), y)=(-1)^{\ell-1}(-1)^{\ell} \operatorname{sign}(\rho(x), y) \operatorname{sign}(\rho(y), x)
$$

so $\prod_{(b, w) \in \rho} \operatorname{sign}(b, w)$ changes. Since $\operatorname{sign}_{B W}(\rho)$ changes, the sign of the LHS of $(2.1 .2)$ does not change sign.

Case 3. If neither $x$ nor $y$ is paired with a black node in the interval $[x+1, x+2, \ldots, y-1]$, then $(-1)^{\#}$ crosses of $\rho$ changes sign. By Lemma 2.1.23,

$$
\operatorname{sign}(\rho(x), x) \operatorname{sign}(\rho(y), y)=\left((-1)^{\ell}\right)^{2} \operatorname{sign}(\rho(x), y) \operatorname{sign}(\rho(y), x)
$$

so $\prod_{(b, w) \in \rho} \operatorname{sign}(b, w)$ does not change. Since $\operatorname{sign}_{B W}(\rho)$ changes, the sign of the LHS of (2.1.2) changes.

This completes the proof of Lemma 2.1.13. We conclude Section 2.1 by proving Lemma 2.1.3, which states that when a black-white pairing $\rho$ is also odd-even, $\operatorname{sign}_{O E}(\rho)=\operatorname{sign}_{B W}(\rho)$. 
2.1.3. Proof of Lemma 2.1.3. Before we prove Lemma 2.1.3, we prove the lemma in the case where $\rho$ is planar.

Lemma 2.1.27. When $\rho$ is a planar black-white pairing,

$$
\operatorname{sign}_{O E}(\rho)=\operatorname{sign}_{B W}(\rho)
$$

Proof. Let $\rho$ be a planar black-white pairing. Recall from Definition 2.1.16 that all black-white pairings can be written $\rho=\left(\left(b_{1}, \rho\left(b_{1}\right)\right),\left(b_{2}, \rho\left(b_{2}\right)\right), \ldots,\left(b_{n}, \rho\left(b_{n}\right)\right)\right)$, where $b_{1}<b_{2}<\cdots<b_{n}$, and we say that $\left(\rho\left(b_{i}\right), \rho\left(b_{j}\right)\right)$ is an inversion of $\rho$ if $i<j$ and $\rho\left(b_{i}\right)>\rho\left(b_{j}\right)$.

All planar pairings are odd-even, and recall from Definition 2.1.17 that if

$\rho=((1, \rho(1)),(3, \rho(3)), \ldots,(2 n-1, \rho(2 n-1)))$ is an odd-even pairing, we say $(\rho(i), \rho(j))$ is an inversion if $i<j$ and $\rho(i)>\rho(j)$.

We will show that there is a one-to-one correspondence between inversions of $\rho$ when it is considered as a black-white pairing (which we will call black-white inversions) and inversions of $\rho$ when it is considered as an odd-even pairing (which we will call odd-even inversions).

Consider a black-white inversion, that is, some $b_{i}<b_{j}$ such that $\rho\left(b_{i}\right)>\rho\left(b_{j}\right)$. There are several cases to consider:

Case 1. $b_{i}, b_{j}$ are both odd.

In this case, $b_{i}=2 k-1$ and $b_{j}=2 \ell-1$ for some $k<\ell$, so $\left(\rho\left(b_{i}\right), \rho\left(b_{j}\right)\right)$ is an odd-even inversion.

Case 2. $b_{i}, b_{j}$ are both even.

Since $b_{i}<b_{j}$ and $\rho\left(b_{i}\right)>\rho\left(b_{j}\right),\left(b_{j}, b_{i}\right)$ is an odd-even inversion.

Case 3. $b_{i}$ is odd and $b_{j}$ is even.

There are two subcases to consider. If $\rho\left(b_{j}\right)<b_{i}$, then it must be the case that $b_{j}>\rho\left(b_{i}\right)$. To see this, observe that if $b_{j}<\rho\left(b_{i}\right)$, then $\rho\left(b_{j}\right)<b_{i}<b_{j}<\rho\left(b_{i}\right)$, but then we have a crossing, which contradicts the planarity of $\rho$. So $\left(b_{j}, \rho\left(b_{i}\right)\right)$ is an odd-even inversion.

If $\rho\left(b_{j}\right)>b_{i}$, then $\rho\left(b_{i}\right)>b_{j}$ (otherwise $b_{i}<\rho\left(b_{j}\right)<\rho\left(b_{i}\right)<b_{j}$, so $\rho$ has a crossing). So $\left(\rho\left(b_{i}\right), b_{j}\right)$ is an odd-even inversion.

Case 4. $b_{i}$ is even and $b_{j}$ is odd.

If $\rho\left(b_{i}\right)>b_{j}$, then $\rho\left(b_{j}\right)>b_{i}$ (otherwise $\rho\left(b_{j}\right)<b_{i}<b_{j}<\rho\left(b_{i}\right)$ is a crossing), so $\left(\rho\left(b_{j}\right), b_{i}\right)$ is an odd-even inversion. If $\rho\left(b_{i}\right)<b_{j}$, then $b_{i}>\rho\left(b_{j}\right)$ (otherwise $b_{i}<\rho\left(b_{j}\right)<\rho\left(b_{i}\right)<b_{j}$ is a crossing), so $\left(b_{i}, \rho\left(b_{j}\right)\right)$ is an odd-even inversion.

A similar argument shows that for each odd-even inversion, there is a black-white inversion. Since there is a one-to-one correspondence between odd-even inversions and black-white inversions, $\operatorname{sign}_{O E}(\rho)=\operatorname{sign}_{B W}(\rho)$.

Lemma 2.1.3. When $\rho$ is a black-white pairing that is also odd-even,

$$
\operatorname{sign}_{O E}(\rho)=\operatorname{sign}_{B W}(\rho) .
$$

Proof. One can get from an odd-even black-white pairing $\rho_{1}$ to any other odd-even black white pairing $\rho_{2}$ by applying a series of moves, where each move swaps the locations of two nodes of the same color and parity. Since each of these moves changes $\operatorname{sign}_{O E}$ and $\operatorname{sign}_{B W}$, the claim follows from Lemma 2.1.27.

2.2. Lemmas 3.1 and $\mathbf{3 . 2}$ from Kenyon and Wilson. Throughout this section, $S$ denotes a balanced subset of nodes (a subset containing an equal number of black and white nodes). In [KW11a], Kenyon and Wilson show that $Z^{D}(G \backslash S) Z^{D}\left(G \backslash S^{c}\right)$ is a sum of double-dimer partition 
functions $Z_{\pi}^{D D}(G, \mathbf{N})$, where the sum is over all pairings $\pi$ that do not connect nodes in $S$ to nodes in $S^{c}$.

Lemma 2.2.1. [KW11a, Lemma 3.1] If $S$ is a balanced subset of nodes then $Z^{D}(G \backslash S) Z^{D}\left(G \backslash S^{c}\right)$ is a sum of double-dimer configurations for all connection topologies $\pi$ for which $\pi$ connects no element of $S$ to an element of $S^{c}:=\mathbf{N} \backslash S$. That is,

$$
Z^{D}(G \backslash S) Z^{D}\left(G \backslash S^{c}\right)=Z^{D D} \sum_{\pi} M_{S, \pi} \operatorname{Pr}(\pi)
$$

where $M_{S, \pi}$ is 0 or 1 according to whether $\pi$ connects nodes in $S$ to $S^{c}$ or not.

This lemma relates the quantity $Z^{D}(G \backslash S) Z^{D}\left(G \backslash S^{c}\right)$ to $\operatorname{Pr}(\pi)$. Next, Kenyon and Wilson show that $\frac{Z^{D}(G \backslash S) Z^{D}\left(G \backslash S^{c}\right)}{\left(Z^{D}(G)\right)^{2}}$ is a determinant in the quantities $X_{i, j}$.

Lemma 2.2.2. [KW11a, Lemma 3.2] Let $S$ be a balanced subset of $\{1, \ldots, 2 n\}$. Then

$$
\frac{Z^{D}(G \backslash S) Z^{D}\left(G \backslash S^{c}\right)}{\left(Z^{D}(G)\right)^{2}}=\operatorname{det}\left[\left(1_{i, j \in S}+1_{i, j \notin S}\right) \times(-1)^{(|i-j|-1) / 2} X_{i, j}\right]_{j=2,4, \ldots, 2 n}^{i=1,3, \ldots, 2 n-1} .
$$

The combination of these results shows that $\widehat{\operatorname{Pr}}(\pi)$ is a homogeneous polynomial in the $X_{i, j}$, since the matrix $M$ from [KW11a, Lemma 3.1] has full rank [KW11a, Lemma 3.3]. Our analogues of these lemmas have several differences (such as the additional global signs in our version of Lemma 2.2.2, see Lemma 2.2.5), but our proofs are quite similar to their proofs.

We begin with Lemma 2.2.1. For a graph $G$ with node set $\mathbf{N}$ that does not necessarily have the property that all nodes are black and odd or white and even, a statement very similar to Lemma 2.2.1 holds. For the remainder of this section, we let $T \subseteq \mathbf{N}$ be the set of nodes that are odd and white or even and black. Since $\mathbf{N}$ is assumed to have an equal number of black and white nodes, $|T|$ is even.

Let $\widetilde{G}$ be $G$ with an extra vertex and edge with weight 1 added to each node in $T$, so all of the nodes in $\widetilde{G}$ are black and odd or white and even. We note that $Z^{D}(\widetilde{G} \backslash S)=Z^{D}(G \backslash(S \triangle T))$, where $S \triangle T$ denotes the symmetric difference of the sets $S$ and $T$. For example, if $\mathbf{N}$ is a set of 12 nodes colored so that nodes $1,3,4,5,7$, and 10 are black (see the proof of Lemma 2.1.14) then $T=\{4,9,10,11\}$. If $S=\{2,3,9,10\}$, then $S \triangle T=\{2,3,4,11\}$. Lemma 2.2 .1 implies the following.

Corollary 2.2.3. Let $S$ be a balanced subset of nodes. $Z^{D}(G \backslash(S \triangle T)) Z^{D}\left(G \backslash(S \triangle T)^{c}\right)$ is a sum of double-dimer configurations for all connection topologies $\pi$ for which $\pi$ connects no element of $S$ to an element of $S^{c}$. That is,

$$
Z^{D}(G \backslash(S \triangle T)) Z^{D}\left(G \backslash(S \triangle T)^{c}\right)=Z^{D D}(G) \sum_{\pi} M_{S, \pi} \operatorname{Pr}(\pi),
$$

where $M_{S, \pi}$ is 0 or 1 according to whether $\pi$ connects nodes in $S$ to $S^{c}$ or not.

If $V=S \triangle T$, then $S=V \triangle T$, so we have:

Corollary 2.2.4. Let $V$ be a balanced subset of nodes. $Z^{D}(G \backslash V) Z^{D}\left(G \backslash V^{c}\right)$ is a sum of all connection topologies $\pi$ for which $\pi$ connects no elements of $V \triangle T$ to $(V \triangle T)^{c}$. That is,

$$
Z^{D}(G \backslash V) Z^{D}\left(G \backslash V^{c}\right)=Z^{D D}(G) \sum_{\pi} M_{V \triangle T, \pi} \operatorname{Pr}(\pi),
$$

where $M_{V \triangle T, \pi}$ is 0 or 1 depending on whether $\pi$ connects nodes in $V \triangle T$ to $(V \triangle T)^{c}$.

Corollary 2.2.4 is the version of Lemma 2.2.1 that we will need to prove Theorem 1.3.1.

Our version of [KW11a, Lemma 3.2] is the following. 
Lemma 2.2.5. Let $S$ be a balanced subset of $\mathbf{N}=\{1, \ldots, 2 n\}$. Then

$$
\frac{Z^{D}(G \backslash S) Z^{D}\left(G \backslash S^{c}\right)}{\left(Z^{D}(G)\right)^{2}}=\operatorname{sign}_{c}(\mathbf{N}) \operatorname{sign}(S) \operatorname{det}\left[\left(1_{i, j \in S}+1_{i, j \notin S}\right) \times \operatorname{sign}(i, j) Y_{i, j}\right]_{j=w_{1}, w_{2}, \ldots, w_{n}}^{i=b_{1}, b_{2}, \ldots, b_{n}}
$$

where $b_{1}, b_{2}, \ldots, b_{n}$ are the black nodes of $\{1,2, \ldots, 2 n\}$ listed in ascending order, $w_{1}, w_{2}, \ldots, w_{n}$ are the white nodes of $\{1,2, \ldots, 2 n\}$ listed in ascending order, sign $(i, j)$ is defined in Definition 2.1.6, $Y_{i, j}=\frac{Z^{D}\left(G_{i, j}\right)}{Z^{D}(G)}$, and

$$
\operatorname{sign}(S)=(-1)^{\# \text { crosses of } \rho,}
$$

where $\rho$ is a black-white pairing that does not connect ${ }^{6} S$ to $S^{c}$ and is planar when restricted to $S$ and planar when restricted to $S^{c}$.

Remark 2.2.6. The fact that such a pairing $\rho$ always exists is a consequence of Lemma 2.1.14, which states that for any node coloring there is a planar black-white pairing $\rho$ satisfying $\operatorname{sign}_{B W}(\rho) \prod_{(b, w) \in \rho} \operatorname{sign}(b, w)=\operatorname{sign}_{c}(\mathbf{N})$. Since $S$ is balanced, the existence of a planar black-white pairing of $S$ and a planar black-white pairing of $S^{c}$ follows.

The proof of Lemma 2.2.5 requires some Kasteleyn theory. The reader familiar with basic facts about Kasteleyn matrices can skip the following section.

2.2.1. Kasteleyn matrices. Recall that $G=\left(V_{1}, V_{2}, E\right)$ is a finite edge-weighted bipartite planar graph embedded in the plane. Let $\omega((i, j))$ denote the weight of an edge $(i, j) \in E$.

Definition 2.2.7. A Kasteleyn (or flat) weighting of $G$ is a choice of sign for each edge with the property that each face with 0 mod 4 edges has an odd number of - signs and each face with 2 mod 4 edges has an even number of - signs.

For the remainder of this section we will let $\sigma: E \rightarrow \pm 1$ denote the Kasteleyn weighting of $G$. A Kasteleyn matrix of $G$ is a weighted, signed bipartite adjacency matrix of $G$. More precisely, define a $\left|V_{1}\right| \times\left|V_{2}\right|$ matrix $K$ by

$$
K_{i, j}= \begin{cases}\sigma((i, j)) \omega((i, j)) & \text { if }(i, j) \in E \\ 0 & \text { otherwise }\end{cases}
$$

Kasteleyn showed that every bipartite planar graph with an even number of vertices has a Kasteleyn matrix [Kas67]. Furthermore, if $\left|V_{1}\right|=\left|V_{2}\right|$ then $|\operatorname{det} K|$ is the weighted sum of all dimer configurations of $G$.

The proof of Lemma 2.2.5 uses a few straightforward facts about Kasteleyn weightings. First, we will show that if $G=\left(V_{1}, V_{2}, E\right)$ has a Kasteleyn weighting $\sigma$, and we add edges to $G$ to obtain $G^{\prime}$, we can choose weights for the added edges to obtain a Kasteleyn weighting $\sigma^{\prime}$ of $G^{\prime}$ with the property that $\sigma^{\prime}(e)=\sigma(e)$ for all $e \in E$.

Lemma 2.2.8. Let $b$ and $w$ be two vertices of opposite color on a face $F$ of $G=\left(V_{1}, V_{2}, E\right)$. Let $E^{\prime}=E \cup\{\tilde{e}\}$, where $\tilde{e} \notin E$ is an edge connecting $b$ and $w$ that separates Finto two faces and let $G^{\prime}=\left(V_{1}, V_{2}, E^{\prime}\right)$. Define $\sigma^{\prime}: E \cup\{\tilde{e}\} \rightarrow \pm 1$ so that $\sigma^{\prime}(e)=\sigma(e)$ for all $e \in E$ and choose $\sigma^{\prime}(\tilde{e})$ so that one of the faces bounded by $\tilde{e}$ is flat (i.e., it has an odd number of - signs if it has 0 mod 4 edges, and an even number of - signs otherwise). Then $\sigma^{\prime}$ is a Kasteleyn weighting of $G^{\prime}$.

\footnotetext{
$S^{c "}$.

${ }^{6}$ The statement " $\rho$ does not connect $S$ to $S^{c}$ " is an abbreviation for " $\rho$ does not connect nodes in $S$ to nodes in
} 
Proof. By assumption, the edge $\widetilde{e}$ separates $F$ into two faces: the face consisting of the edges of a path $\mathcal{Q}$ and the edge $\widetilde{e}$, and the face consisting of the edges of a path $\mathcal{P}$ and the edge $\widetilde{e}$. The path $\mathcal{P}$ consists of $1 \bmod 4$ edges or $3 \bmod 4$ edges. Define

$$
\sigma^{\prime}(\tilde{e})= \begin{cases}\prod_{e \in \mathcal{P}} \sigma(e) & \text { if } \mathcal{P} \text { has } 1 \bmod 4 \text { edges } \\ -\prod_{e \in \mathcal{P}} \sigma(e) & \text { if } \mathcal{P} \text { has } 3 \bmod 4 \text { edges }\end{cases}
$$

and define $\sigma^{\prime}(e)=\sigma(e)$ for all $e \in E$. Now the face consisting of the path $\mathcal{P}$ and the edge $e$ is flat. It remains to check that the face $F^{\prime}$ consisting of $\mathcal{Q}$ and $\widetilde{e}$ is flat, which is done by breaking into cases based on whether the paths $\mathcal{P}, \mathcal{Q}$ have 1 or 3 edges mod 4 .

Lemma 2.2.9. Let $W=\left\{v_{1}, \ldots, v_{2 m}\right\}$ be a set of vertices on the outer face of $G=\left(V_{1}, V_{2}, E\right)$. Pair the vertices of $W$ so that we can add edges $e_{1}, \ldots, e_{m}$ connecting the pairs without introducing any edge crossings. Let $E^{(m)}=E \cup\left\{e_{1}, \ldots, e_{m}\right\}$ and let $G^{(m)}=\left(V_{1}, V_{2}, E^{(m)}, \omega\right)$. Define $\sigma_{i}$ : $E \cup\left\{e_{i}\right\} \rightarrow \pm 1$ as in Lemma 2.2.8: $\sigma_{i}(e)=\sigma(e)$ for all $e \in E$ and $\sigma_{i}\left(e_{i}\right)$ is chosen so that one of the faces bounded by $e_{i}$ is flat. By Lemma 2.2.8, $\sigma_{i}$ is a Kasteleyn weighting for all $1 \leq i \leq m$. Then $\tau: E \cup\left\{e_{1}, \ldots, e_{m}\right\} \rightarrow \pm 1$ defined by $\tau(e)=\sigma(e)$ for all $e \in E$ and $\tau\left(e_{i}\right)=\sigma_{i}\left(e_{i}\right)$ for $1 \leq i \leq m$ is a Kasteleyn weighting of $G^{(m)}$.

Proof. We prove the claim by induction on $m$. When $m=1$, there is nothing to show. Assume the claim holds when we add $m-1$ edges to $G$. Now suppose we add $m$ edges $e_{1}, \ldots, e_{m}$. Choose an "innermost" edge $e_{j}$, i.e. an edge with the property that one of its faces is bounded only by edges of $G$ and $e_{j}$. By the induction hypothesis, $\tau: E \cup\left\{e_{1}, \ldots, e_{j-1}, e_{j+1}, \ldots, e_{m}\right\} \rightarrow \pm 1$ defined by $\tau(e)=\sigma(e)$ for all $e \in E$ and $\tau\left(e_{i}\right)=\sigma_{i}\left(e_{i}\right)$ for $i=1,2, \ldots, j-1, j+1, \ldots, m$ is a Kasteleyn weighting of $G^{(m-1)}=\left(V_{1}, V_{2}, E \cup\left\{e_{1}, \ldots, e_{j-1}, e_{j+1}, \ldots e_{m}\right\}\right)$. Since $e_{j}$ is an innermost edge and $\sigma_{j}: E \cup\left\{e_{j}\right\} \rightarrow \pm 1$ was defined so that when $e_{j}$ is added to $G$, one of the faces bounded by $e_{j}$ is flat, we may apply Lemma 2.2 .8 to conclude that $\tau: E \cup\left\{e_{1}, \ldots, e_{m}\right\} \rightarrow \pm 1$ defined by $\tau(e)=\sigma(e)$ for all $e \in E$ and $\tau\left(e_{i}\right)=\sigma_{i}\left(e_{i}\right)$ for $1 \leq i \leq m$ is a Kasteleyn weighting of $G^{(m)}=\left(V_{1}, V_{2}, E \cup\left\{e_{1}, \ldots, e_{m}\right\}\right)$.

We also need to show that if we delete rows and columns from a Kasteleyn matrix of a graph, the resulting submatrix is a Kasteleyn matrix of the corresponding graph.

Lemma 2.2.10. Let $K$ be a Kasteleyn matrix of $G$. Let $S$ be a balanced subset of vertices on the outer face of $G$. Then $K_{\backslash S}$, the submatrix of $K$ formed by deleting the rows and columns from $S$, is a Kasteleyn matrix of $G \backslash S$.

To prove this, we need the following lemma and corollary, which are proven in [Kup94].

Lemma 2.2.11. [Kup94, Theorem 2.1] If $G$ is a planar bipartite graph with an even number of vertices, there are an even number of faces with $4 k$ sides.

Corollary 2.2.12. [Kup94, Theorem 2.2] Every signed graph with an even number of vertices has an even number of non-flat faces.

Proof of Lemma 2.2.10. $G \backslash S$ is flat at each internal face because $G$ is flat at each internal face, so it remains to show that it is flat on the outer face as well. Since $G \backslash S$ has an even number of vertices, it has an even number of non-flat faces by Corollary 2.2.12, so it must be flat on the outer face.

\subsubsection{Proof of Lemma 2.2.5.}

Proof of Lemma 2.2.5. Assume there are $2 k$ couples of consecutive nodes of the same color. As in Remark 2.1.7, we label the couples of consecutive white nodes $\left(u_{i}, u_{i}+1\right)$ and the couples of consecutive black nodes $\left(s_{i}, s_{i}+1\right)$ for $1 \leq i \leq k$. 
Following the proof of [KW11a, Lemma 3.2], we adjoin to the graph $G 2 n-2 k$ edges connecting all adjacent nodes except nodes $s_{i}$ and $s_{i}+1$ and nodes $u_{i}$ and $u_{i}+1$. The resulting graph is still bipartite by the assumption that the nodes alternate between black and white except for the nodes $s_{i}$ and $s_{i}+1$ and the nodes $u_{i}$ and $u_{i}+1$. Now add $4 k$ more edges as follows. Since $G$ is bipartite, there is a white vertex $t_{i}$ on the outer face of $G$ between nodes $s_{i}$ and $s_{i}+1$ and a black vertex $v_{i}$ on the outer face of $G$ between nodes $u_{i}$ and $u_{i}+1$. Add edges connecting nodes $s_{i}$ and $t_{i}$ and $t_{i}$ and $s_{i}+1$, and edges connecting nodes $u_{i}$ and $v_{i}$ and $v_{i}$ and $u_{i}+1$. Give the $2 n-2 k+4 k=2 n+2 k$ edges we have added weight $\epsilon$ (and then take the limit $\epsilon \rightarrow 0$ ). Let $G^{\prime}$ denote the resulting graph.

Given a Kasteleyn weighting of a graph, the signs of edges incident to a vertex may be reversed, and each face will still have a correct number of minus signs. Fix a Kasteleyn weighting of the graph $G^{\prime}$. List the vertices from the set $\mathbf{N} \cup\left\{t_{i}\right\}_{i=1}^{k} \cup\left\{v_{i}\right\}_{i=1}^{k}$ in counterclockwise order. For each vertex in this list, if the edge from the vertex $i$ to the next vertex in the list $j$ has a minus sign, reverse the signs of all edges incident to vertex $j$. This ensures that the edges of weight $\epsilon$ we added to $G$ have positive sign, with the possible exception of the edge from node $2 n$ to 1 , which must have sign $-(-1)^{n+k}$ for the outer face to have a correct number of minus signs (because if $n+k$ is even, the outer face has $0 \bmod 4$ edges, and if $n+k$ is odd, the outer face has $2 \bmod 4$ edges).

Let $S$ be a balanced subset of $\{1, \ldots, 2 n\}$. Let $\left(w_{1}, b_{1}\right), \ldots,\left(w_{j}, b_{j}\right)$ be any noncrossing pairing of the nodes of $S$, where $w_{1}, \ldots, w_{j}$ are the white nodes of $S$ and $b_{1}, \ldots, b_{j}$ are the black nodes of $S$. Adjoin edges of weight $W$ connecting $w_{i}$ to $b_{i}$ for $1 \leq i \leq j$. Because of the edges of weight $\epsilon$ we adjoined to $G$, we let the sign of a new edge of weight $W$ connecting black node $b$ and white node $w$ be

$$
\operatorname{sign}(b, w)=(-1)^{\left(|b-w|+a_{b, w}-1\right) / 2},
$$

where recall that $a_{b, w}$ is the number of couples of consecutive nodes of the same color in the interval $[\min \{b, w\}, \min \{b, w\}+1, \ldots, \max \{b, w\}]$.

Observe that with this choice of signs, when we add any one of the edges $e_{i}=\left(b_{i}, w_{i}\right)$ to $G^{\prime}$ so that it separates the outer face of $G^{\prime}$ into two faces, one of the faces bounded by $e_{i}$ is flat. So by Lemma 2.2.9, this is a Kasteleyn weighting.

Let $K_{W}$ be the Kasteleyn matrix of the resulting graph, with rows and columns ordered so that $b_{1}, \ldots, b_{j}$ are the first $j$ rows and $w_{1}, \ldots, w_{j}$ are the first $j$ columns. Let $K=K_{0}$ be the corresponding Kasteleyn matrix when $W=0$. Then $Z^{D}(G \backslash S)= \pm\left[W^{j}\right] \operatorname{det}\left(K_{W}\right)$ where $\left[W^{j}\right] \operatorname{det}\left(K_{W}\right)$ denotes the coefficient of $W^{j}$ in the polynomial $\operatorname{det}\left(K_{W}\right)$. (Because $\left[W^{j}\right] \operatorname{det}\left(K_{W}\right)$ is, up to a sign, the weighted sum of matchings that include all of the edges of weight $W$, which is exactly the weighted sum of matchings of $G \backslash S$.) Since each term of $\operatorname{det}\left(K_{W}\right)$ has the same sign,

$$
\frac{Z^{D}(G \backslash S)}{Z^{D}(G)}=\frac{\left[W^{j}\right] \operatorname{det}\left(K_{W}\right)}{\left[W^{0}\right] \operatorname{det}\left(K_{W}\right)}
$$

Next let $K_{\backslash S}$ denote the submatrix of $K$ formed by deleting the rows and columns from $S$. By Lemma 2.2.10, $K_{\backslash S}$ is a Kasteleyn matrix of $G \backslash S$. The sign of $\operatorname{det}\left(K_{\backslash S}\right)$ and the sign of $\left[W^{j}\right] \operatorname{det}\left(K_{W}\right)$ differ by the product of the signs of the edges of weight $W$. So, noting that $\left[W^{0}\right] \operatorname{det}\left(K_{W}\right)=\operatorname{det}(K)$, we have

$$
\frac{\left[W^{j}\right] \operatorname{det}\left(K_{W}\right)}{\left[W^{0}\right] \operatorname{det}\left(K_{W}\right)}=\prod_{\ell=1}^{j} \operatorname{sign}\left(b_{\ell}, w_{\ell}\right) \frac{\operatorname{det}\left(K_{\backslash S}\right)}{\operatorname{det}(K)} .
$$

By Jacobi's determinant identity,

$$
\prod_{\ell=1}^{j} \operatorname{sign}\left(b_{\ell}, w_{\ell}\right) \frac{\operatorname{det}\left(K_{\backslash S}\right)}{\operatorname{det}(K)}=\prod_{\ell=1}^{j} \operatorname{sign}\left(b_{\ell}, w_{\ell}\right) \operatorname{det}\left[K_{b, w}^{-1}\right]_{w=w_{1}, \ldots, w_{j}}^{b=b_{1}, \ldots, b_{j}}
$$


So we have

$$
\frac{Z^{D}(G \backslash S)}{Z^{D}(G)}=\prod_{\ell=1}^{j} \operatorname{sign}\left(b_{\ell}, w_{\ell}\right) \operatorname{det}\left[K_{b, w}^{-1}\right]_{w=w_{1}, \ldots, w_{j}}^{b=b_{1}, \ldots, b_{j}} .
$$

Letting $S=\{b, w\}$ in equation (2.2.2), we get

$$
Y_{b, w}=\frac{Z^{D}\left(G_{b, w}\right)}{Z^{D}(G)}=\operatorname{sign}(b, w) K_{b, w}^{-1} .
$$

From this and equation $(2.2 .2)$ we find that when $\rho_{1}=\left(w_{1}, b_{1}\right), \ldots,\left(w_{j}, b_{j}\right)$ is a noncrossing pairing of the nodes of $S$ and $\rho_{2}=\left(w_{j+1}, b_{j+1}\right), \ldots,\left(w_{n}, b_{n}\right)$ is a noncrossing pairing of the nodes of $S^{c}$,

$$
\begin{aligned}
& \frac{Z^{D}(G \backslash S) Z^{D}\left(G \backslash S^{c}\right)}{\left(Z^{D}(G)\right)^{2}} \\
= & \prod_{\ell=1}^{j} \operatorname{sign}\left(b_{\ell}, w_{\ell}\right) \prod_{\ell=j+1}^{n} \operatorname{sign}\left(b_{\ell}, w_{\ell}\right) \operatorname{det}\left[\operatorname{sign}(b, w) Y_{b, w}\right]_{w=w_{1}, \ldots, w_{j}}^{b=b_{1}, \ldots, b_{j}} \operatorname{det}\left[\operatorname{sign}(b, w) Y_{b, w}\right]_{w=w_{j+1}, \ldots, w_{n}}^{b=b_{j+1}, \ldots, b_{n}} \\
= & \prod_{\ell=1}^{n} \operatorname{sign}\left(b_{\ell}, w_{\ell}\right) \operatorname{det}\left[\left(1_{i, j \in S}+1_{i, j \notin S}\right) \operatorname{sign}(b, w) Y_{b, w}\right]_{w=w_{1}, \ldots, w_{n}}^{b=b_{1}, \ldots, b_{n}}
\end{aligned}
$$

which is equation (2.2.1), except for the global sign and the order of the rows and columns (since $w_{1}, \ldots, w_{n}$ and $b_{1}, \ldots, b_{n}$ are not necessarily in ascending order).

Let $\rho=\rho_{1} \cup \rho_{2}$. Reorder the rows so that the black nodes are in ascending order. For each row swap, make the corresponding column swap. Then $\rho$ pairs the node corresponding to row $i$ with the node corresponding to column $i$. Since the row swaps and column swaps we have made are in one-to-one correspondence, we have not changed the sign of the determinant. Finally, we need to put the columns in ascending order. The number of swaps required to do this is exactly $\operatorname{sign}_{B W}(\rho)$.

So after reordering the rows and columns so that they are listed in ascending order, the global sign is:

$$
\prod_{\ell=1}^{n} \operatorname{sign}\left(b_{\ell}, w_{\ell}\right) \operatorname{sign}_{B W}(\rho)
$$

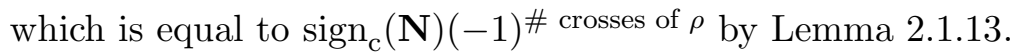

2.3. Defining $\mathcal{Q}^{(D D)}$. Let $Y^{\prime}$ be the vector of monomials $Y_{\rho}^{\prime}$ indexed by black-white pairings, where $Y_{\rho}^{\prime}=(-1)^{\# \text { crosses of } \rho} \prod_{(i, j) \in \rho} Y_{i, j}$.

In this section, we define $Q^{(D D)}$, which is the matrix satisfying $P=Q^{(D D)} Y^{\prime}$, where $P$ is the vector indexed by planar pairings $\pi$ with entries $\widetilde{\operatorname{Pr}}(\pi)$. Recall from Section 1.3 that $\widetilde{\operatorname{Pr}}(\pi)=$ $\frac{Z_{\pi}^{D D}(G, \mathbf{N})}{\left(Z^{D}(G)\right)^{2}}$

We begin with a few definitions.

Definition 2.3.1. If $\sigma$ and $\tau$ are two pairings on a set of nodes $\{1,2, \ldots, 2 n\}$, construct the undirected multigraph $C$ with vertex set $\{1,2, \ldots, 2 n\}$ by adding an edge between vertices $i$ and $j$ for each pair $(i, j)$ of $\sigma$, and similarly for $\tau$. The number of components in $\sigma \cup \tau$ is the number of connected components in $C$. Note that all connected components of $C$ are cycles. 
Example 2.3.2. If $\sigma=((1,2),(3,4),(5,6))$ and $\tau=((1,5),(2,6),(3,4))$ then there are two components in $\sigma \cup \tau$, as shown below.

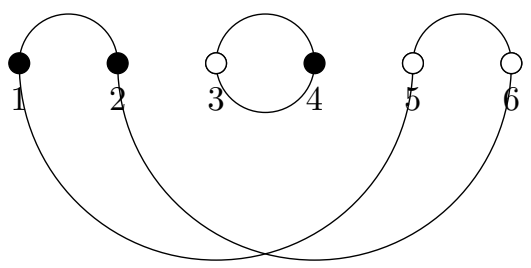

Definition 2.3.3. If $\pi$ is an odd-even pairing and $\rho$ is a black-white pairing, define

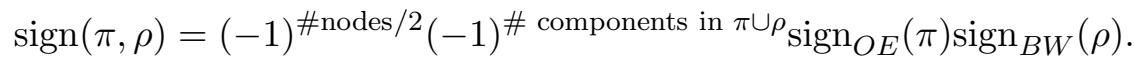

Definition 2.3.4. Define the matrix $\mathcal{B}_{2}$ which has rows indexed by planar pairings and columns indexed by black-white pairings by

$$
\left(\mathcal{B}_{2}\right)_{\pi, \rho}=\operatorname{sign}(\pi, \rho) 2^{\# \text { components in } \pi \cup \rho} \text {. }
$$

Let $M$ be the matrix from Corollary 2.2.4 and let $D$ be the vector indexed by balanced sets $S$ with entries $D_{S}=\frac{Z^{D}(G \backslash S) Z^{D}\left(G \backslash S^{c}\right)}{\left(Z^{D}(G)\right)^{2}}$ (see Lemma 2.2.5). Following Kenyon and Wilson, we will show that

$$
M^{T} D=\mathcal{B}_{2} Y^{\prime}
$$

(Lemma 2.3.5). This result is nontrivial, requiring several lemmas, but once it is established it is nearly immediate that

$$
M^{T} M P=\mathcal{B}_{2} Y^{\prime}
$$

where $P$ is the vector indexed by planar pairings $\pi$ with entries $\widetilde{\operatorname{Pr}}(\pi)$ (Theorem 2.3.9). Kenyon and Wilson proved that $M^{T} M$ is invertible ([KW09, Theorem 3.3]), so we conclude the section by defining $\mathcal{Q}^{(D D)}$ as $\left(M^{T} M\right)^{-1} \mathcal{B}_{2}$.

Lemma 2.3.5 (analogue of Lemma 3.5 from [KW11a]). $M^{T} D=\mathcal{B}_{2} Y^{\prime}$.

In the proof of [KW11a, Lemma 3.5], Kenyon and Wilson use the fact that if the nodes of $G$ are all either black and odd or white and even and $\pi$ and $\rho$ are odd-even pairings, then there are

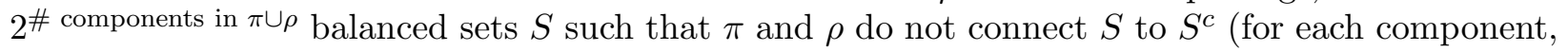
either put all of its nodes in $S$ or all of its nodes in $S^{c}$ ). Recall from Section 2.2 that $T \subseteq \mathbf{N}$ is the set of nodes that are odd and white or even and black; under Kenyon and Wilson's assumptions, $T=\emptyset$. It turns out that after removing the requirement that the nodes be black and odd or white and even, if $\pi$ is an odd-even pairing and $\rho$ is a black-white pairing there are still $2^{\#}$ components in $\pi \cup \rho$ sets $S$ such that $\rho$ does not connect $S$ to $S^{c}$ and $\pi$ does not connect $S \triangle T$ to $(S \triangle T)^{c}$.

Lemma 2.3.6. Let $\pi$ be an odd-even pairing and let $\rho$ be a black-white pairing. For each component of $\pi \cup \rho$ there are exactly two ways to put the nodes in this component into $S$ and $S^{c}$ so that $\rho$ does not connect $S$ to $S^{c}$ and $\pi$ does not connect $S \triangle T$ to $(S \triangle T)^{c}$.

Proof. We start by placing an initial node $a$ into $S$ or $S^{c}$, and then apply the algorithm below until all nodes in the component have been placed into $S$ or $S^{c}$.

\section{Algorithm}

Step 1

(a) If $a \in S \cap T^{c}$ or $a \in S^{c} \cap T$ :

(i) If $\pi(a) \in T$ :

Put $\pi(a)$ in $S^{c}$. 
(ii) Else if $\pi(a) \notin T$ :

Put $\pi(a)$ in $S$.

(b) Else if $a \in S \cap T$ or $a \in S^{c} \cap T^{c}$ :

(i) If $\pi(a) \in T$ :

Put $\pi(a)$ in $S$.

(ii) Else if $\pi(a) \notin T$ :

Put $\pi(a)$ in $S^{c}$.

Go to Step 2 with $a:=\pi(a)$.

Step 2
If $a \in S$ :
Put $\rho(a)$ in $S$.
Else if $a \in S^{c}$ :
Put $\rho(a)$ in $S^{c}$.
Go to Step 1 with $a:=\rho(a)$.

Claim. The set $S$ described in the algorithm is well-defined and balanced.

Proof. We will prove this claim by induction on the number of nodes in a component of $\pi \cup \rho$.

Base Cases. First note that in the case where the nodes alternate between black and white, $T=\emptyset$

or $T=\mathbf{N}$ so the algorithm reduces to putting all of the nodes in a component in $S$ or all of the nodes of a component in $S^{c}$, so $S$ is well-defined. Since in this case both pairings are black-white, $S$ is balanced as well.

If there are two nodes in a component, since $\rho$ is a black-white pairing one of the nodes is black and the other is white, so by the previous comment there is nothing to show.

If there are four nodes in a component, since $\rho$ is a black-white pairing two nodes must be black and two nodes must be white. By symmetry, it is enough to consider when nodes 1 and 2 are black and nodes 3 and 4 are white. There are two odd-even pairings: $((1,2),(3,4))$ and $((1,4),(3,2))$ and two black-white pairings: $((1,4),(3,2))$ and $((1,3),(2,4))$.

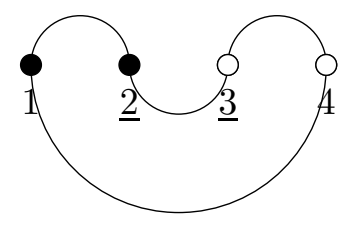

Figure 6. The diagram of $\pi \cup \rho$ when $\pi=((1,2),(3,4))$ and $\rho=((1,4),(2,3))$. The nodes in $T$ are underlined. If we start the algorithm by putting $1 \in S$, we get $S=\{1,4\}$.

For example, when $\pi=((1,2),(3,4))$ and $\rho=((1,4),(2,3))$ (see Figure 6 ), we start by putting node 1 in $S$. (We could also start by putting node 1 in $S^{c}$.) Then we run the algorithm:

Step 1. Since $1 \notin T$ and $\pi(1)=2 \in T$, we put $2 \in S^{c}$.

Step 2. Since $2 \in S^{c}$ we put $\rho(2)=3 \in S^{c}$.

Step 1 . Since $3 \in S^{c}, 3 \in T$ and $4 \notin T$, we put $4 \in S$.

So we get $S=\{1,4\}$, which is balanced. To check that $S$ is well-defined, it suffices to show that if we continue the algorithm for one more step, we do not get a contradiction. If we apply Step 2 starting at node 4 , we get that we should put $\rho(4)=1$ in $S$, as desired.

In the table below are the results of applying the algorithm for each possible combination of odd-even pairings $\pi$ and black-white pairings $\rho$ that results in a component of size 4 . When $\pi=\rho=((1,4),(3,2))$, there are two components each of size 2 , so this is omitted from the table. 


\begin{tabular}{c|c|c|c|c|c}
$\pi$ & $\rho$ & $S$ & start & end & one more step \\
\hline$((1,2),(3,4))$ & $((1,4),(2,3))$ & $\{1,4\}$ & $1 \in S$ & $4 \in S$ & $1 \in S$ \\
$((1,2),(3,4))$ & $((1,3),(2,4))$ & $\{1,3\}$ & $1 \in S$ & $3 \in S$ & $1 \in S$ \\
$((1,4),(3,2))$ & $((1,3),(2,4))$ & $\{1,2,3,4\}$ & $1 \in S$ & $3 \in S$ & $1 \in S$
\end{tabular}

In each case, $S$ is balanced, and continuing the algorithm for one more step does not create a contradiction.
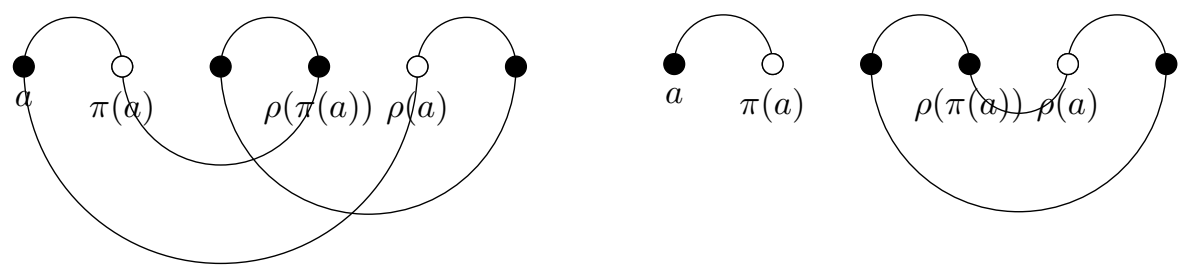

Figure 7. Illustration of case 1. Shown left is the odd-even pairing $\pi$ (top) and the black-white pairing $\rho$ (bottom). On the right we have replaced $\rho$ with $\tilde{\rho}$, the black white pairing on $\mathbf{N}-$ $\{a, \pi(a)\}$ obtained by pairing $\rho(\pi(a))$ with $\rho(a)$.

Now suppose that a component of $\pi \cup \rho$ has $2 n$ nodes, where $2 n>4$. Assume that if a component has fewer than $2 n$ nodes, the set $S$ is well-defined and balanced. Let $\mathbf{N}$ denote the set of nodes in this component. There are two cases to consider based on whether or not $\left.\pi\right|_{\mathbf{N}}$ has a black-white pair.

Case 1. (Illustrated in Figure 7). Assume $\left.\pi\right|_{\mathbf{N}}$ has at least one black-white pair $(a, \pi(a))$. Since $\rho$ is a black-white pairing, $\rho(a)$ and $\rho(\pi(a))$ are opposite color. Consider the black-white pairing $\tilde{\rho}$ on $\mathbf{N}-\{a, \pi(a)\}$ obtained from $\rho$ by removing the pairs $(a, \rho(a))$ and $(\pi(a), \rho(\pi(a)))$ and adding the pair $(\rho(a), \rho(\pi(a)))$. Let $\tilde{\pi}=\left.\pi\right|_{\mathbf{N}-\{a, \pi(a)\}}$. Now $\tilde{\pi} \cup \tilde{\rho}$ is a single component with $2 n-2$ nodes. Start the algorithm by putting $\rho(\pi(a)) \in S$. By the induction hypothesis, the set $S$ produced by the algorithm is well-defined and balanced. Note that the fact that $S$ is well-defined means that $\rho(a) \in S$.

Considering the original component of $\pi \cup \rho$, when we start the algorithm at $\rho(\pi(a))$ it proceeds identically as it did with $\tilde{\pi} \cup \tilde{\rho}$ until we reach the node $\rho(a)$. Since $\rho(a) \in S$, applying Step 2 of the algorithm we add $a$ to $S$. (Note that we are guaranteed to be on Step 2 here by the fact that $\rho(a)$ is paired with $\rho(\pi(a))$ in $\tilde{\rho}$, and the algorithm starts with Step 1.) Since $\pi$ is odd-even, black-white pairs of $\pi$ have the property that either both nodes are in $T$ or both are not in $T$. So after the next step of the algorithm (Step 1) we add $\pi(a)$ to $S$. Since we added $a$ and $\pi(a)$ to $S, S$ is still balanced. Since $\pi(a) \in S$, continuing the algorithm for one more step would put $\rho(\pi(a)) \in S$, which is consistent.

Case 2. (Illustrated in Figure 8). If $\left.\pi\right|_{\mathbf{N}}$ does not have a black-white pair, then consider a white pair of $\left.\pi\right|_{\mathbf{N}}:(a, \pi(a))$. Let $b=\rho(a)$. Since $a$ is white, $\rho(a)$ must be black, and $(b, \pi(b))$ is a black pair of $\left.\pi\right|_{\mathbf{N}}$ by the assumption that $\left.\pi\right|_{\mathbf{N}}$ does not have a black-white pair. Consider the black-white pairing $\tilde{\rho}$ on $\mathbf{N}-\{a, \pi(a), b, \pi(b)\}$ obtained from $\rho$ by removing the pairs $(a, b),(\pi(a), \rho(\pi(a)))$, and $(\pi(b), \rho(\pi(b)))$ and adding the pair $(\rho(\pi(a)), \rho(\pi(b)))$. Let $\tilde{\pi}=\left.\pi\right|_{\mathbf{N}-\{a, \pi(a), b, \pi(b)\}}$. Now $\tilde{\pi} \cup \tilde{\rho}$ is a single component with $2 n-4$ nodes. Start the algorithm by putting $\rho(\pi(a)) \in S$. By the induction hypothesis, the set $S$ produced by the algorithm is well-defined and balanced. Note that the fact that $S$ is well defined means that $\rho(\pi(b)) \in S$.

Considering the original component of $\pi \cup \rho$, when we start the algorithm by putting $\rho(\pi(a)) \in S$ it proceeds identically as it did with $\tilde{\pi} \cup \tilde{\rho}$ until we reach the node $\rho(\pi(b))$. Since $\rho(\pi(b)) \in S$, applying Step 2 of the algorithm we add $\pi(b)$ to $S$. Since $\pi$ is odd-even, exactly one of $\{b, \pi(b)\}$ is in $T$. This means that after applying Step 1 we put $b \in S^{c}$. Then we put $a \in S^{c}($ since $\rho(a)=b)$ 


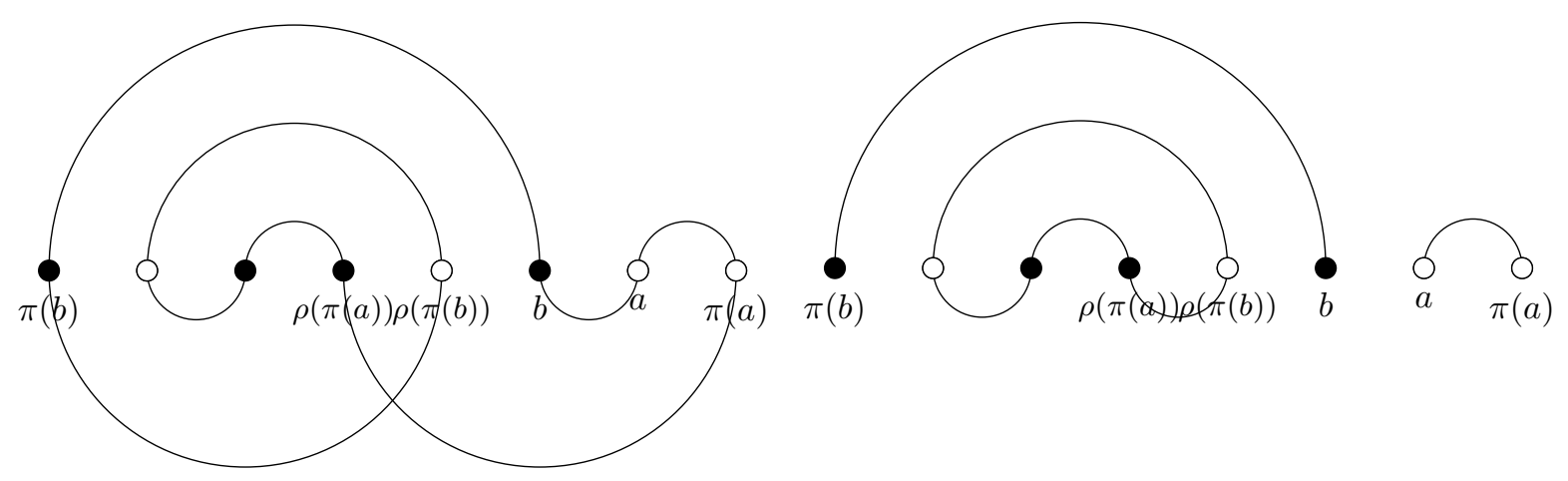

Figure 8. Illustration of case 2. Shown left is the odd-even pairing $\pi$ (top) and the black-white pairing $\rho$ (bottom). On the right we have replaced $\rho$ with $\tilde{\rho}$, the black white pairing on $\mathbf{N}-$ $\{a, \pi(a), b, \pi(b)\}$ obtained by pairing $\rho(\pi(a))$ with $\rho(\pi(b))$.

and $\pi(a) \in S$ (since exactly one of $\{a, \pi(a)\}$ is in $T$ ). Since we added $\pi(b)$ and $\pi(a)$ to $S, S$ is still balanced. Since $\pi(a) \in S$, continuing the algorithm for one more step puts $\rho(\pi(a)) \in S$, which is consistent.

Claim. After applying this algorithm, $\rho$ does not connect $S$ to $S^{c}$ and $\pi$ does not connect $S \triangle T$ to $(S \triangle T)^{c}$.

Proof. By Step 2, for each node $a, a$ and $\rho(a)$ will either both be in $S$ or $S^{c}$, so $\rho$ does not connect $S$ to $S^{c}$. To show that $a$ and $\pi(a)$ are either both in $S \triangle T$ or both in $(S \triangle T)^{c}$, there are several cases to consider.

- If $a$ and $\pi(a)$ are both not in $T$, then they are both placed into $S$ by Step 1(a)(ii) or both placed into $S^{c}$ by Step 1(b)(ii). In the first case, $a$ and $\pi(a)$ are both in $S \triangle T$, and in the second case $a$ and $\pi(a)$ are both in $(S \triangle T)^{c}$.

- If $a \in T$ and $\pi(a) \notin T$, then one of $a, \pi(a)$ is placed into $S$ and one is placed into $S^{c}$ by Step 1(a)(ii) or Step 1(b)(ii). If $a$ is placed into $S$ and $\pi(a)$ is placed into $S^{c}$, then $a$ and $\pi(a)$ are both in $(S \triangle T)^{c}$. The other case is similar.

- If $a \in T$ and $\pi(a) \in T$, then they are both placed into $S^{c}$ by Step 1(a)(i) or both placed into $S$ by Step 1(b)(i).

- If $a \notin T$ and $\pi(a) \in T$, then one is placed in $S$ and one is placed in $S^{c}$ by Step 1(a)(i) or Step 1(b)(i).

We have shown that the algorithm produces a well-defined balanced set $S$ with the desired properties. We conclude that for each component of $\pi \cup \rho$ there are exactly two ways to put the nodes in this component into $S$ and $S^{c}$ so that $\rho$ does not connect $S$ to $S^{c}$ and $\pi$ does not connect $S \triangle T$ to $(S \triangle T)^{c}$.

We need two more facts to prove Lemma 2.3.5.

Lemma 2.3.7. Let $S$ be a balanced subset of nodes and let $\operatorname{sign}(S)$ be defined as in Lemma 2.2.5. Then

$$
\operatorname{sign}(S)=(-1)^{\frac{\# \text { nodes }}{2}}(-1)^{\# \text { comp in } \pi \cup \rho} \operatorname{sign}_{O E}(\pi) \operatorname{sign}_{B W}(\rho),
$$

where $\pi$ is an odd-even pairing such that $\pi$ does not connect $S \triangle T$ to $(S \triangle T)^{c}$ and $\rho$ is a black-white pairing such that $\rho$ does not connect $S$ to $S^{c}$. 
The proof of Lemma 2.3.7 is lengthy and technical so we postpone it to Section 2.6 for ease of exposition. The following is an immediate consequence of this lemma.

Corollary 2.3.8. Let $\pi$ be an odd-even pairing and let $\rho$ be a black-white pairing. If $S_{1}$ and $S_{2}$ are balanced subsets of nodes such that $\pi$ does not connect $S_{i} \triangle T$ to $\left(S_{i} \triangle T\right)^{c}$ and $\rho$ does not connect $S_{i}$ to $S_{i}^{c}$ for $i=1,2$, then $\operatorname{sign}\left(S_{1}\right)=\operatorname{sign}\left(S_{2}\right)$.

To see that Corollary 2.3.8 follows from Lemma 2.3.7, observe that if $\pi$ and $\rho$ satisfy the hypotheses of Lemma 2.3.7 for $S_{1}$ and $S_{2}$, then $S_{1}$ and $S_{2}$ must have the same sign, because all of the quantities on the right hand side of the equation in Lemma 2.3.7 depend only on $\pi$ and $\rho$.

Proof of Lemma 2.3.5. Recall from Lemma 2.2.5 that

$$
D_{S}=\frac{Z^{D}(G \backslash S) Z^{D}\left(G \backslash S^{c}\right)}{\left(Z^{D}(G)\right)^{2}}=\operatorname{sign}_{\mathrm{c}}(\mathbf{N}) \operatorname{sign}(S) \operatorname{det}\left[\left(1_{i, j \in S}+1_{i, j \notin S}\right) \times \operatorname{sign}(i, j) Y_{i, j}\right]_{j=w_{1}, w_{2}, \ldots, w_{n}}^{i=b_{1}, b_{2}, \ldots, b_{n}},
$$

where $b_{1}, b_{2}, \ldots, b_{n}$ are the black nodes listed in ascending order and $w_{1}, w_{2}, \ldots, w_{n}$ are the white nodes listed in ascending order.

When we expand the determinant, we get

$$
D_{S}=\operatorname{sign}_{\mathrm{c}}(\mathbf{N}) \operatorname{sign}(S) \sum_{\substack{\text { BW pairings } \rho: \\ \rho \text { does not connect } \\ S \text { to } S^{c}}} \operatorname{sign}_{B W}(\rho) \prod_{(i, j) \in \rho} \operatorname{sign}(i, j) Y_{i, j} .
$$

By Lemma 2.1.13,

$$
D_{S}=\operatorname{sign}_{\mathrm{c}}(\mathbf{N}) \operatorname{sign}(S) \sum_{\substack{\text { BW pairings } \rho: \\ \rho \text { does not connect } \\ S \text { to } S^{c}}} \operatorname{sign}_{\mathrm{c}}(\mathbf{N})(-1)^{\# \text { crosses of } \rho} \prod_{(i, j) \in \rho} Y_{i, j},
$$

and thus by definition,

$$
D_{S}=\operatorname{sign}(S) \sum_{\substack{\text { BW pairings } \rho: \\ \rho \text { does not connect } \\ S \text { to } S^{c}}} Y_{\rho}^{\prime} .
$$

Let $\pi$ be a planar pairing and let $M$ be the matrix from Corollary 2.2.4. The $\pi$ th row of $M^{T} D$ is

$$
\sum_{\substack{S \subseteq\{1,2, \ldots, 2 n\} \\ \text { does not connect } \\ S \triangle T \text { to }(S \triangle T)^{c}}} D_{S}
$$

We see that

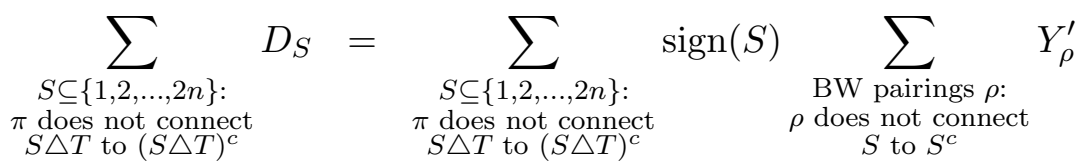

$$
\begin{aligned}
& =\sum_{\text {BW pairings } \rho} \sum_{\begin{array}{c}
S: \rho \text { does not } \\
\text { connect } S \text { to } S^{c} \text { and } \\
\pi \text { does not } \operatorname{connect} \\
S \triangle T \text { to }(S \triangle T)^{c}
\end{array}} \operatorname{sign}(S) Y_{\rho}^{\prime} .
\end{aligned}
$$

By Lemma 2.3.6 and Corollary 2.3.8,

$$
\sum_{\text {BW pairings } \rho} \sum_{\substack{\left.S: \rho \text { does not } \\ \text { connect } S \text { to } S^{c} \text { and } \\ \pi \text { does not connect } \\ S \triangle T \text { to } S \triangle T\right)^{c}}} \operatorname{sign}(S) Y_{\rho}^{\prime}=\sum_{\text {BW pairings } \rho} \operatorname{sign}(\pi, \rho) 2^{\# \text { comp in } \pi \cup \rho} Y_{\rho}^{\prime} .
$$


Since this sum is the $\pi$ th row of $\mathcal{B}_{2} Y^{\prime}$, we have proven the claim.

Theorem 2.3.9 (analogue of Theorem 3.6 from [KW11a]). $M^{T} M P=\mathcal{B}_{2} Y^{\prime}$

Proof. Noting that $\widetilde{\operatorname{Pr}}(\pi)=\operatorname{Pr}(\pi) Z^{D D}(G) /\left(Z^{D}(G)\right)^{2}$, we see that by Corollary 2.2.4, MP $=D$. Then, applying Lemma 2.3.5 we have $M^{T} M P=M^{T} D=\mathcal{B}_{2} Y^{\prime}$.

It remains to show that $M^{T} M$ is invertible. In fact, $M^{T} M$ is equal to the meander matrix $\mathcal{M}_{q}$ evaluated at $q=2$.

Lemma 2.3.10. [KW11a, Lemma 3.3] Let $M$ be the matrix from Lemma 2.2.1. Then $M^{T} M=\mathcal{M}_{2}$, where $\mathcal{M}_{2}$ is a matrix with rows and columns indexed by planar pairings, with entries

$$
\left(\mathcal{M}_{2}\right)_{\sigma, \tau}=2^{\# \text { comp in } \sigma \cup \tau}
$$

Since the only difference between the matrix from Lemma 2.2.1 and the matrix from Corollary 2.2.4 is the ordering of the rows, Lemma 2.3.10 applies to the matrix $M$ from Corollary 2.2.4 as well.

Definition 2.3.11. Since $\mathcal{M}_{2}$ is invertible (see [DFGG97]), define

$$
\mathcal{Q}^{(D D)}=\mathcal{M}_{2}^{-1} \mathcal{B}_{2}
$$

Since $P=\mathcal{Q}^{(D D)} Y^{\prime}, \mathcal{Q}^{(D D)}$ is the matrix of the $Y^{\prime}$ polynomials: for a given planar pairing $\pi$, the $\pi$ th row of $\mathcal{Q}^{(D D)}$ gives the polynomial $\widetilde{\operatorname{Pr}}(\pi)$.

That is,

$$
\widetilde{\operatorname{Pr}}(\sigma)=\sum_{\text {black-white pairings } \rho} \mathcal{Q}_{\sigma, \rho}^{(D D)} Y_{\rho}^{\prime}
$$

Our next aim is to prove that $\mathcal{Q}^{(D D)}$ is integer-valued. To this end, we will show that we can compute the columns combinatorially using a transformation rule from Kenyon and Wilson's study of groves [KW11a].

\subsection{Groves.}

Definition 2.4.1. [KW11a] If $G$ is a finite edge-weighted planar graph embedded in the plane with a set of nodes, a grove is a spanning acyclic subgraph of $G$ such that each component tree contains at least one node. The weight of a grove is the product of the weights of the edges it contains.

The connected components of a grove partition the nodes into a planar partition. If $\sigma$ is a planar partition of $1,2, \ldots, n$, let $\operatorname{Pr}(\sigma)$ be the probability that a random grove of $G$ partitions the nodes according to $\sigma$. Kenyon and Wilson showed that $\dddot{\operatorname{Pr}}(\sigma):=$ $\frac{\operatorname{Pr}(\sigma)}{\operatorname{Pr}(1|2| \cdots \mid n)}$ is an integer-coefficient homogeneous polynomial in the variables $L_{i, j}{ }^{7}$ [KW11a, Theorem 1.2].

For example, the normalized probability $\dddot{\operatorname{Pr}}(\sigma)$ that a random grove on four nodes partitions the nodes according to $1 \mid 234$ is $\dddot{\operatorname{Pr}}(\sigma)=L_{2,3} L_{3,4}+L_{2,3} L_{2,4}+L_{2,4} L_{3,4}+L_{1,3} L_{2,4}$. (See [KW11a, Section 1.2]).

Each monomial in the polynomial $\dddot{\operatorname{Pr}}(\sigma)$ is of the form $L_{\tau}=$ $\sum_{F} \prod_{\{i, j\} \in F} L_{i, j}$. The sum is over spanning forests $F$ of the complete

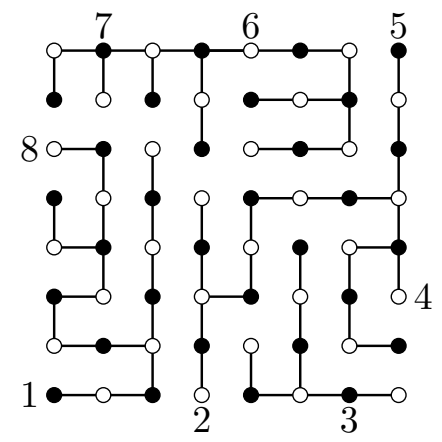

Figure 9. A grove of a grid graph with 8 nodes. The partition of the nodes is $\{\{1,8\},\{2,4,5\},\{3\},\{6,7\}\}$.

\footnotetext{
${ }^{7}$ When $G$ is viewed as a resistor network with conductances equal to the edge weights, $L_{i, j}$ is the current that would flow into node $j$ if node $i$ were set to one volt and all other nodes were set to zero volts [KW11a, Appendix A].
} 
graph $K_{n}$ for which the trees of $F$ span the parts of $\tau$ and the product is over edges $\{i, j\}$ of the forest $F$.

To compute these polynomials, Kenyon and Wilson define a matrix $\mathcal{P}^{(t)}$ with rows indexed by planar partitions and columns indexed by all partitions and show how to compute the columns of this matrix combinatorially. The $\tau$ th column of $\mathcal{P}^{(t)}$ is computed by writing the partition $\tau$ as a linear combination of planar partitions. So if $\tau$ is planar, then $\mathcal{P}_{\tau, \tau}^{(t)}=1$ and $\mathcal{P}_{\sigma, \tau}^{(t)}=0$ for all $\sigma \neq \tau$. If $\tau$ is nonplanar, the rule is a generalization of the rule for four nodes:

$$
13|24 \rightarrow 1| 234+2|314+3| 124+4|123-12| 34-14 \mid 23
$$

This rule tells us, for example, that $P_{12|34,13| 24}^{(t)}=-1$.

In general, if a partition is nonplanar, then there will exist nodes $a<b<c<d$ such that $a$ and $c$ belong to one part, and $b$ and $d$ belong to another part. In Kenyon and Wilson's transformation rule, $1,2,3$, and 4 in equation (2.4.1) are replaced with parts $A, B, C$ and $D$, which contain the nodes $a, b, c$ and $d$, respectively.

Rule 2.4.2. [KW11a, Rule 1] Arbitrarily subdivide the part containing $a$ and $c$ into two sets $A$ and $C$ such that $a \in A$ and $c \in C$, and similarly subdivide the part containing $b$ and $d$ into $B \ni b$ and $D \ni d$. Let the remaining parts of the partition be denoted by "rest." Then the transformation rule is

$A C|B D|$ rest $\rightarrow A|B C D|$ rest $+B|A C D|$ rest $+C|A B D|$ rest $+D|A B C|$ rest $-A B|C D|$ rest $-A D|B C|$ rest

Remark 2.4.3. If we arbitrarily subdivide the part containing $a$ and $c$ into two sets $A$ and $C$ such that $a \in A$ and $c \in C$, and similarly for the part containing $b$ and $d$, it is possible to repeat Rule 2.4.2 indefinitely without ever obtaining a linear combination of planar partitions.

For example, consider the partition $1235 \mid 46$. One crossing is $a=1, b=4, c=5, d=6$. If we choose $A=\{1,2,3\}, B=\{4\}, C=\{5\}$, and $D=\{6\}$, then after applying Rule 2.4.2, all of the partitions are planar. But if we choose $A=\{1,2\}, B=\{4\}, C=\{3,5\}$, and $D=\{6\}$ then after applying Rule 2.4 .2 we get

$$
12|3456+4| 12356+35|1246+6| 12345-124|356-126| 345
$$

which includes nonplanar partitions. For example, the partition 124|356 has crossing $a=1, b=3$, $c=4, d=6$. If we choose $A=\{1,2\}, B=\{3,5\}, C=\{4\}$, and $D=\{6\}$ then after applying Rule 2.4 .2 to $124 \mid 356$ we get

$$
12|3456+35| 1246+4|12356+6| 12345-1235|46-126| 345 .
$$

So after applying Rule 2.4.2 twice, all partitions cancel except for the partition 1235|46, which is the partition we started with. We could continue this process indefinitely.

Remark 2.4.3 motivates the following modification of Rule 2.4.2.

Rule 2.4.4. Subdivide the part containing $a$ and $c$ into two sets $A$ and $C$ such that $A$ contains all the items in this part less than $b$, and $C$ contains all other items. Similarly, subdivide the part containing $b$ and $d$ into two sets $B$ and $D$ so that $B$ contains all items in this part less than $c$, and $D$ contains all other items. Then the transformation rule is

$A C|B D|$ rest $\rightarrow A|B C D|$ rest $+B|A C D|$ rest $+C|A B D|$ rest $+D|A B C|$ rest $-A B|C D|$ rest $-A D|B C|$ rest

Applying Rule 2.4.4 repeatedly will result in a linear combination of planar partitions.

We have now presented all the definitions needed to state Kenyon and Wilson's main result for groves. 
Theorem 2.4.5. [KW11a, Theorem 1.2] Any partition $\tau$ may be transformed into a formal linear combination of planar partitions by repeated application of Rule 2.4.4. ${ }^{8}$, and the resulting linear combination does not depend on the choices made when applying Rule 2.4.4, so that we may write

$$
\tau \rightarrow \sum_{\text {planar partitions } \sigma} \mathcal{P}_{\sigma, \tau}^{(t)} \sigma .
$$

For any planar partition $\sigma$, the same coefficients $\mathcal{P}_{\sigma, \tau}^{(t)}$ satisfy the equation

$$
\dddot{\operatorname{Pr}}(\sigma)=\frac{\operatorname{Pr}(\sigma)}{\operatorname{Pr}(1|2| 3|\cdots| n)}=\sum_{\text {partitions } \tau} \mathcal{P}_{\sigma, \tau}^{(t)} L_{\tau}
$$

for bipartite edge-weighted planar graphs.

2.5. Proof that $\mathcal{Q}^{D D}$ is integer-valued. We will complete the proof of Theorem 1.3.1 by showing that we can use the transformation rule introduced in the previous section to compute the columns of the matrix $\mathcal{Q}^{(D D)}$.

Remark 2.5.1. For pairings, both Rule 2.4 .2 and Rule 2.4 .4 become the following: If a pairing $\rho$ is nonplanar, then there will exist items $a<b<c<d$ such that $a$ and $c$ are paired, and $b$ and $d$ are paired. Then the transformation rule is

$$
a c|b d| \text { rest } \rightarrow-a b|c d| \text { rest }-a d|b c| \text { rest. }
$$

Rule 2.5.2. For a black-white pairing $\rho$, repeatedly apply (2.5.1) until we have written $\rho$ as a linear combination of planar pairings. Then multiply each planar pairing in this sum by $\operatorname{sign}_{O E}(\sigma) \operatorname{sign}_{B W}(\rho)$.

The fact that Rule 2.5.2 is well-defined follows from Theorem 2.4.5. Proving that Rule 2.5.2 computes the columns of $\mathcal{Q}^{(D D)}$ will prove that the matrix $\mathcal{Q}^{(D D)}$ is integer-valued and gives us the desired theorem, which is stated in full below.

Theorem 1.3.1. Any black-white pairing $\rho$ can be transformed into a formal linear combination of planar pairings by repeated application of Rule 2.5.2, and the resulting linear combination does not depend on the choices we made when applying Rule 2.5.2, so that we may write

$$
\rho \rightarrow \sum_{\text {planar pairings } \sigma} \mathcal{Q}_{\sigma, \rho}^{(D D)} \sigma .
$$

For any planar pairing $\sigma$, these same coefficients $\mathcal{Q}_{\sigma, \rho}^{(D D)}$ satisfy the equation

$$
\widetilde{\operatorname{Pr}}(\sigma):=\frac{Z_{\sigma}^{D D}(G, \mathbf{N})}{\left(Z^{D}(G)\right)^{2}}=\sum_{\text {black-white pairings } \rho} \mathcal{Q}_{\sigma, \rho}^{(D D)} Y_{\rho}^{\prime} .
$$

Remark 2.5.3. The fact that the resulting linear combination does not depend on the choices we made when applying Rule 2.5.2 is an immediate consequence of Theorem 2.4.5.

The proof of Theorem 1.3.1 requires two additional lemmas.

Lemma 2.5.4. Let $\pi$ be a pairing and let $\rho$ be a pairing with nodes $a<b<c<d$ that form $a$ crossing in $\rho$. Let $\rho_{1}$ be the pairing obtained from $\rho$ by replacing the pairs $(a, c)$ and $(b, d)$ with $(a, b)$ and $(c, d)$ and let $\rho_{2}$ be the pairing obtained from $\rho$ by replacing the pairs $(a, c)$ and $(b, d)$ with $(a, d)$ and $(b, c)$. Then either

(1) $\pi \cup \rho$ has one more component than both $\pi \cup \rho_{1}$ and $\pi \cup \rho_{2}$,

(2) $\pi \cup \rho_{1}$ has one more component than $\pi \cup \rho$, and $\pi \cup \rho_{2}$ and $\pi \cup \rho$ have the same number of components, or

\footnotetext{
${ }^{8}$ In [KW11a, Theorem 1.2], Rule 2.4.2 is used in the theorem statement, but for the reasons stated in Remark 2.4.3, we have changed it to Rule 2.4.4.
} 
(3) $\pi \cup \rho_{2}$ has one more component than $\pi \cup \rho$, and $\pi \cup \rho_{1}$ and $\pi \cup \rho$ have the same number of components.

Proof. Observe that either $a, b, c$, and $d$ are all in the same component of $\pi \cup \rho$ or $a$ and $c$ are in the same component and $b$ and $d$ are in a different component. If $a$ and $c$ are in the same component and $b$ and $d$ are in a different component, then pairing $a$ with $b$ and $c$ with $d$ merges these two components. Similarly, pairing $a$ with $d$ and $b$ with $c$ merges these two components.

If $a, b, c$ and $d$ are in the same component, then we consider the following path in $\pi \cup \rho$ :

$$
c-a-\pi(a)-\rho(\pi(a))-\cdots
$$

This path reaches $b$ or $d$ before it reaches $c$ since by assumption $a, b, c, d$ are all in the same component. If it reaches $b$ before $d$, then in $\rho_{1}, a$ and $b$ are in a different component than $c$ and $d$. This is because path (2.5.2) is replaced with

$$
b-a-\pi(a)-\rho(\pi(a))-\cdots-b,
$$

so $\pi \cup \rho_{1}$ has one more component than $\pi \cup \rho$. In $\rho_{2}, a, b, c$, and $d$ are all in the same component, because path $(2.5 .2)$ is replaced with

$$
d-a-\pi(a)-\rho(\pi(a))-\cdots-b-c,
$$

so $\pi \cup \rho_{2}$ and $\pi \cup \rho$ have the same number of components. If the path reaches $d$ before $b$, then in $\rho_{2}, a$ and $d$ are in a different component than $b$ and $c$, so $\pi \cup \rho_{2}$ has one more component than $\pi \cup \rho$. In $\rho_{1}, a, b, c$, and $d$ are in the same component, so $\pi \cup \rho_{1}$ and $\pi \cup \rho$ have the same number of components.

Lemma 2.5.5. Let $\rho$ be a pairing (not necessarily black-white). Then for any planar pairing $\pi$,

$$
\operatorname{sign}_{O E}(\pi)(-1)^{C_{\rho}}(-1)^{\# \text { nodes } / 2} 2^{C_{\rho}}=\sum_{\text {planar pairings } \sigma} \mathcal{P}_{\sigma, \rho}^{(t)} \operatorname{sign}_{O E}(\sigma) 2^{C_{\sigma}} .
$$

Here, $C_{\rho}$ denotes the number of components in $\pi \cup \rho$ and $C_{\sigma}$ denotes the number of components in $\pi \cup \sigma$.

Proof. We will prove the claim by induction on the number of crossings in $\rho$.

Base Case. When $\rho$ has 0 crossings, equation (2.5.3) becomes

$$
\operatorname{sign}_{O E}(\pi)(-1)^{C_{\rho}}(-1)^{\# \text { nodes } / 2} 2^{C_{\rho}}=\mathcal{P}_{\rho, \rho}^{(t)} \operatorname{sign}_{O E}(\rho) 2^{C_{\rho}}
$$

which is equivalent to

$$
\operatorname{sign}_{O E}(\pi)(-1)^{C_{\rho}}(-1)^{\# \text { nodes } / 2} \operatorname{sign}_{O E}(\rho)=1 .
$$

First suppose $\rho=\pi$. Since $(-1)^{\# ~ c o m p ~ i n ~} \pi \cup \pi=(-1)^{\# \text { nodes } / 2}$, equation (2.5.4) holds. We can obtain any planar pairing from any other planar pairing by a sequence of moves, where each move consists of swapping the locations of two nodes of the same parity. So we will show that when $\rho$ is a planar pairing, $x$ and $y$ are two nodes of the same parity, and $\rho^{\prime}$ is the pairing obtained from $\rho$ by swapping the locations of $x$ and $y$, replacing $\rho$ with $\rho^{\prime}$ does not change the left hand side of equation (2.5.4).

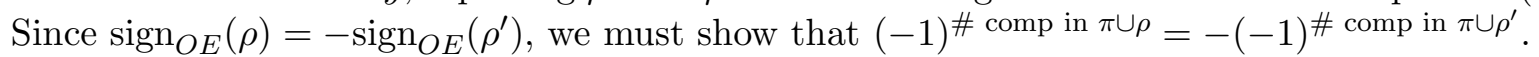

If $x$ and $\rho(x)$ are in a different component than $y$ and $\rho(y)$ in $\pi \cup \rho$, then $\pi \cup \rho^{\prime}$ has one fewer component than $\pi \cup \rho$. If $x, \rho(x), y$, and $\rho(y)$ are all in the same component in $\pi \cup \rho$, then without loss of generality assume that $x$ and $y$ are both even, so $\rho(x)$ and $\rho(y)$ are both odd, and consider the following path in $\pi \cup \rho$ :

$$
\rho(x)-x-\pi(x)-\rho(\pi(x))-\cdots .
$$

Since $\rho$ and $\pi$ are both odd-even, segments $n \frown \rho(n)$ go from an odd node to an even node. Since $\rho(y)$ is odd and $y$ is even, this means that we must reach the node $\rho(y)$ before the node $y$. Therefore we have the path

$$
\rho(x)-x-\pi(x)-\rho(\pi(x))-\cdots-\rho(y)-y-\cdots
$$


When we replace the pairs $(x, \rho(x)),(y, \rho(y))$ with $(x, \rho(y))$ and $(y, \rho(x))$, this path is replaced with

$$
\rho(y)-x-\pi(x)-\rho(\pi(x))-\cdots-\rho(y)
$$

so $(x, \rho(y))$ and $(y, \rho(x))$ are in different components of $\pi \cup \rho^{\prime}$. We conclude that equation (2.5.4) holds for all planar pairings $\rho$.

Now assume that equation (2.5.3) holds for pairings $\rho$ with $\leq k$ crossings.

Let $\rho$ be a pairing with $k+1$ crossings. Let $a<b<c<d$ be nodes that form a crossing in $\rho$. Let $\rho_{1}$ be the pairing obtained by replacing the pairs $(a, c)$ and $(b, d)$ with $(a, b)$ and $(c, d)$ and let $\rho_{2}$ be the pairing obtained by replacing the pairs $(a, c)$ and $(b, d)$ with $(a, d)$ and $(b, c)$. We claim that both $\rho_{1}$ and $\rho_{2}$ have fewer than $k+1$ crossings. Observe that if a chord connecting two nodes $n_{1}$ and $n_{2}$ crosses the chord connecting $a$ and $b$ in $\rho_{1}$, it also crosses the chord connecting $a$ and $c$ or the chord connecting $b$ and $d$ in $\rho$. Similarly, if a chord connecting two nodes crosses the chord connecting $c$ and $d$ in $\rho_{1}$, it also crosses the chord connecting $a$ and $c$ or the chord connecting $b$ and $d$ in $\rho$. It follows that $\rho_{1}$ has at least one fewer crossing than $\rho$. A similar argument shows that $\rho_{2}$ has at least one fewer crossing than $\rho$. By the induction hypothesis,

$$
\operatorname{sign}_{O E}(\pi)(-1)^{C_{\rho_{1}}}(-1)^{\# \text { nodes } / 2} 2^{C_{\rho_{1}}}=\sum_{\text {planar pairings } \sigma} \mathcal{P}_{\sigma, \rho_{1}}^{(t)} \operatorname{sign}_{O E}(\sigma) 2^{C_{\sigma}}
$$

and

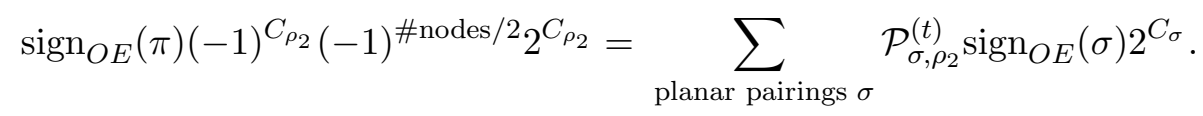

By the transformation rule (2.5.1),

$$
\mathcal{P}_{\sigma, \rho_{1}}^{(t)}+\mathcal{P}_{\sigma, \rho_{2}}^{(t)}=-\mathcal{P}_{\sigma, \rho}^{(t)}
$$

so we have

$$
\sum_{\substack{\text { planar } \\ \text { pairings } \sigma}} \mathcal{P}_{\sigma, \rho}^{(t)} \operatorname{sign}_{O E}(\sigma) 2^{C_{\sigma}}=-\operatorname{sign}_{O E}(\pi)(-1)^{\# \text { nodes } / 2}\left((-1)^{C_{\rho_{1}}} 2^{C_{\rho_{1}}}+(-1)^{C_{\rho_{2}}} 2^{C_{\rho_{2}}}\right) .
$$

By Lemma 2.5.4 there are three cases to consider:

(1) $\pi \cup \rho$ has one more component than both $\pi \cup \rho_{1}$ and $\pi \cup \rho_{2}$,

(2) $\pi \cup \rho_{1}$ has one more component than $\pi \cup \rho$, and $\pi \cup \rho_{2}$ and $\pi \cup \rho$ have the same number of components, and

(3) $\pi \cup \rho_{2}$ has one more component than $\pi \cup \rho$, and $\pi \cup \rho_{1}$ and $\pi \cup \rho$ have the same number of components.

Case (1). Since $C_{\rho_{i}}-C_{\rho}=-1$ for $i=1,2$,

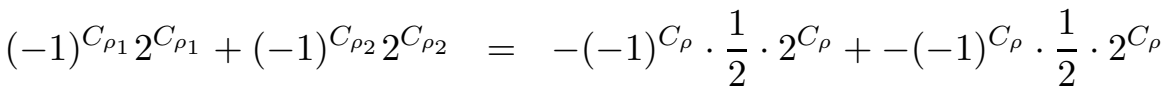

$$
\begin{aligned}
& =(-1)^{C_{\rho}} 2^{C_{\rho}}\left(-\frac{1}{2}-\frac{1}{2}\right) \\
& =-(-1)^{C_{\rho}} 2^{C_{\rho}} \text {. }
\end{aligned}
$$

Cases (2) and (3). We will only include the proof for case (2), since case (3) is completely analogous. Since $C_{\rho_{1}}-C_{\rho}=1$ and $C_{\rho_{2}}-C_{\rho}=0$,

$$
\begin{aligned}
(-1)^{C_{\rho_{1}} 2^{C_{\rho_{1}}}+(-1)^{C_{\rho_{2}}} 2^{C_{\rho_{2}}}} & =-(-1)^{C_{\rho}} 2 \cdot 2^{C_{\rho}}+(-1)^{C_{\rho}} 2^{C_{\rho}} \\
& =(-1)^{C_{\rho}} 2^{C_{\rho}}(-2+1) \\
& =-(-1)^{C_{\rho}} 2^{C_{\rho}} .
\end{aligned}
$$

So in all cases,

$$
-\operatorname{sign}_{O E}(\pi)(-1)^{\# \text { nodes } / 2}\left((-1)^{C_{\rho_{1}}} 2^{C_{\rho_{1}}}+(-1)^{C_{\rho_{2}}} 2^{C_{\rho_{2}}}\right)=\operatorname{sign}_{O E}(\pi)(-1)^{\# \text { nodes } / 2}(-1)^{C_{\rho}} 2^{C_{\rho}},
$$


and thus

$$
\operatorname{sign}_{O E}(\pi)(-1)^{C_{\rho}}(-1)^{\# \text { nodes } / 2} 2^{C_{\rho}}=\sum_{\text {planar pairings } \sigma} \mathcal{P}_{\sigma, \rho}^{(t)} \operatorname{sign}_{O E}(\sigma) 2^{C_{\sigma}}
$$

Proof of Theorem 1.3.1. Let $\widetilde{\mathcal{Q}}$ be the matrix obtained by the procedure from Rule 2.5.2, so the $(\sigma, \rho)$ th entry of $\widetilde{\mathcal{Q}}$ is the product of $\operatorname{sign}_{O E}(\sigma) \operatorname{sign}_{B W}(\rho)$ with the coefficient of $\sigma$ when $\rho$ is written as a linear combination of planar pairings using (2.5.1). That is,

$$
\widetilde{\mathcal{Q}}_{\sigma, \rho}=\operatorname{sign}_{O E}(\sigma) \operatorname{sign}_{B W}(\rho) \mathcal{P}_{\sigma, \rho}^{(t)} .
$$

We will show that

$$
\mathcal{M}_{2} \widetilde{\mathcal{Q}} \mathbf{e}_{i}=\mathcal{B}_{2} \mathbf{e}_{i}
$$

for all $i$. This will show that $\mathcal{M}_{2} \widetilde{\mathcal{Q}}=\mathcal{M}_{2} \mathcal{Q}^{(D D)}$, which proves the theorem since $\mathcal{M}_{2}$ is invertible.

Let $\rho$ be a black-white pairing. Recall from Definition 2.3.4 that $\left(\mathcal{B}_{2}\right)_{\pi, \rho}=\operatorname{sign}(\pi, \rho) 2^{C_{\rho}}$. Then by equation (2.5.5), to show that $\mathcal{M}_{2} \widetilde{\mathcal{Q}} \mathbf{e}_{i}=\mathcal{B}_{2} \mathbf{e}_{i}$, we need to show that for each planar pairing $\pi$,

$$
\operatorname{sign}(\pi, \rho) 2^{C_{\rho}}=\sum_{\text {planar pairings } \sigma} \mathcal{P}_{\sigma, \rho}^{(t)} \operatorname{sign}_{O E}(\sigma) \operatorname{sign}_{B W}(\rho) 2^{C_{\sigma}} .
$$

By Definition 2.3.3,

$$
\operatorname{sign}(\pi, \rho)=(-1)^{\# \text { nodes } / 2}(-1)^{C_{\rho}} \operatorname{sign}_{O E}(\pi) \operatorname{sign}_{B W}(\rho) .
$$

Applying Lemma 2.5.5 completes the proof.

2.6. Another characterization of $\operatorname{sign}(S)$. In this section, we prove Lemma 2.3.7, which was key in establishing Lemma 2.3.5.

Lemma 2.3.7. Let $S$ be a balanced subset of nodes and let $\operatorname{sign}(S)$ be defined as in Lemma 2.2.5. Then

$$
\operatorname{sign}(S)=(-1)^{\# \text { nodes } / 2}(-1)^{\# \text { comp in } \pi \cup \rho} \operatorname{sign}_{O E}(\pi) \operatorname{sign}_{B W}(\rho)
$$

where $\pi$ is an odd-even pairing such that $\pi$ does not connect $S \triangle T$ to $(S \triangle T)^{c}$ and $\rho$ is a black-white pairing such that $\rho$ does not connect $S$ to $S^{c}$.

Proving Lemma 2.3.7 requires

(1) proving that such pairings $\pi$ and $\rho$ always exist,

(2) proving that equation (2.6.1) is well-defined, and

(3) proving that equation (2.6.1) holds.

We will postpone the proof of (1) because the fact that such pairings $\pi$ and $\rho$ always exist will follow quickly from the proofs of (2) and (3).

2.6.1. Proof that equation (2.6.1) is well-defined. The strategy of the proof is to define local moves that allow us to get from a pair $\left(\pi_{1}, \rho_{1}\right)$ such that $\pi_{1}$ does not connect $S \triangle T$ to $(S \triangle T)^{c}$ and $\rho_{1}$ does not connect $S$ to $S^{c}$ to any other pair $\left(\pi_{2}, \rho_{2}\right)$ with this property, and to show that these moves do not change the right hand side of equation (2.6.1).

Specifically, we will define two types of local moves. First, we define moves that modify $\pi$ by swapping the locations of two nodes of the same parity under certain conditions but leave $\rho$ fixed, called moves of type $A_{O E}$. Next, we define moves that modify $\rho$ by swapping the locations of two nodes of the same color under similar conditions but leave $\pi$ fixed, called moves of type $A_{B W}$.

In order to describe the conditions under which we can swap the locations of two nodes, we need the following definition. 
Definition 2.6.1. We call a pair of nodes $(a, \eta(a))$ a transition pair if exactly one of the nodes $a, \eta(a)$ is in $T$.

Remark 2.6.2. If $a$ and $b$ are two nodes in the same component of $\pi \cup \rho$, there are two paths from $a$ to $b$. Since the algorithm in Lemma 2.3.6 is well-defined, the parity of the number of transition pairs is independent of the path.

Definition 2.6.3. Suppose $\pi$ is an odd-even pairing and $\rho$ is a black-white pairing. Let $a$ and $b$ be two nodes of the same parity. If

- $a$ and $b$ are in different components,

- $a$ and $b$ are the same color and a path from $a$ to $b$ contains an even number of transition pairs, or

- $a$ and $b$ are different colors and a path from $a$ to $b$ contains an odd number of transition pairs,

let $\pi^{\prime}$ be the pairing obtained from $\pi$ by swapping the locations of $a$ and $b$ in $\pi$. We say that $\left(\pi^{\prime}, \rho\right)$ and $(\pi, \rho)$ differ by a move of type $A_{O E}$. See Figure 10 for an example.
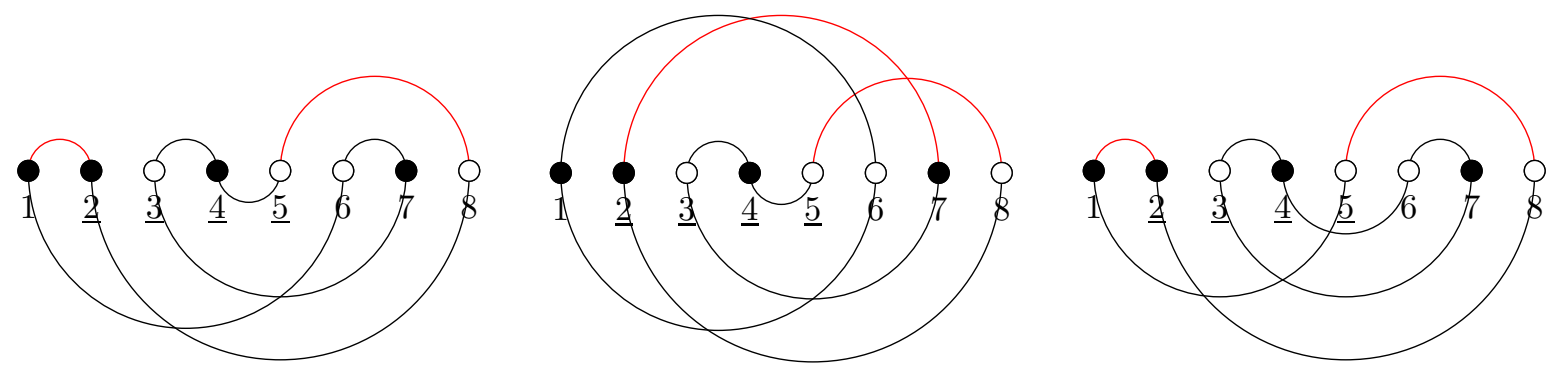

Figure 10. Left: The diagram of $\pi \cup \rho$, where $\pi=((1,2),(3,4),(5,8),(7,6))$ and $\rho=$ $((1,6),(2,8),(4,5),(7,3))$. Nodes that are in $T$ are underlined and arcs between transition pairs are red. Center: Since 1 and 7 are two nodes of the same parity and color and a path from 1 to 7 contains an even number of transition pairs, if we let $\pi^{\prime}=((1,6),(3,4),(5,8),(7,2))$ then $(\pi, \rho)$ and $\left(\pi^{\prime}, \rho\right)$ differ by a move of type $A_{O E}$. Right: Since 1 and 4 are two nodes of the same color and a path from 1 to 4 contains an even number of transition pairs, if we let $\rho^{\prime}=((1,5),(2,8),(4,6),(7,3))$ then $(\pi, \rho)$ and $\left(\pi^{\prime}, \rho\right)$ differ by a move of type $A_{B W}$.

Definition 2.6.4. Let $\pi$ be an odd-even pairing and let $\rho$ be a black-white pairing. Suppose $a$ and $b$ are the same color and either $a$ and $b$ are in different components, or a path in $\pi \cup \rho$ from $a$ to $b$ contains an even number of transition pairs.

Suppose we swap the locations of $a$ and $b$ in $\rho$ to obtain the pairing $\rho^{\prime}$. Then we say that $\left(\pi, \rho^{\prime}\right)$ and $(\pi, \rho)$ differ by a move of type $A_{B W}$. See Figure 10 for an example.

Lemma 2.6.5. Let $\pi, \pi^{\prime}$ be odd-even pairings and let $\rho$ be a black-white pairing such that $(\pi, \rho)$ and $\left(\pi^{\prime}, \rho\right)$ differ by a move of type $A_{O E}$. Then the number of components in $\pi \cup \rho$ and the number of components in $\pi^{\prime} \cup \rho$ differ by one.

Proof. If $a$ and $b$ are in different components of $\pi \cup \rho$, swapping the locations of $a$ and $b$ in $\pi$ merges these two components, so the number of components decreases by one.

If $a$ and $b$ are in the same component, without loss of generality assume that node $a$ is white. Consider the following path from $a$ to $b$, which starts by traversing the edge connecting $a$ to $\pi(a)$ :

$$
a-\pi(a)-\cdots-b \text {. }
$$

We claim that we always reach $b$ before $\pi(b)$. This follows from the observation that because $\rho$ is black-white and $\pi$ is odd-even, a path in $\pi \cup \rho$ alternates between black and white nodes unless a 
pair $(d, \pi(d))$ in the path is a transition pair. So since our path starts at a white node by traversing the edge in $\pi$, if we consider an edge $d \frown \pi(d)$ of the path, $d$ is white and $\pi(d)$ is black if and only if we traverse this edge after passing through an even number of transition pairs. So, if we were to reach $\pi(b)$ before $b, b$ is black if and only if there are an even number of transition pairs between $a$ and $b$, a contradiction since $a$ is white. It follows that we must reach $b$ before $\pi(b)$.

Thus we have the following path in $\pi \cup \rho$ :

$$
a-\pi(a)-\cdots-b-\pi(b)
$$

When we replace the pairs $(a, \pi(a))$ and $(b, \pi(b))$ in $\pi$ with $(a, \pi(b))$ and $(b, \pi(a))$ to obtain $\pi^{\prime}$ the middle portion of the path above $\pi(a)-\cdots-b$ becomes a new component, so the number of components increases by one.

Corollary 2.6.6. A move of type $A_{O E}$ does not change the right hand side of equation (2.6.1).

Proof. If $(\pi, \rho)$ and $\left(\pi^{\prime}, \rho\right)$ differ by a move of type $A_{O E}$, then $(-1)^{\#}$ comp in $\pi \cup \rho=-(-1)^{\#}$ comp in $\pi^{\prime} \cup \rho$ by Lemma 2.6.5 and $\operatorname{sign}_{O E}(\pi)=-\operatorname{sign}_{O E}\left(\pi^{\prime}\right)$, so replacing $\pi$ with $\pi^{\prime}$ does not change the right hand side of equation (2.6.1).

Corollary 2.6.7. A move of type $A_{B W}$ does not change the right hand side of equation (2.6.1).

Proof. The proof that a move of type $A_{B W}$ changes the number of components in $\pi \cup \rho$ by one is analogous to the proof of Lemma 2.6.5. The claim follows as it did in the proof of Corollary 2.6.6.

Proof that equation (2.6.1) is well-defined. By Corollaries 2.6.6 and 2.6.7, moves of type $A_{O E}$ and type $A_{B W}$ do not change the right hand side of equation (2.6.1). So to prove that the formula for $\operatorname{sign}(S)$ is well-defined, it suffices to show that these two types of moves are enough to get from a pair $\left(\pi_{1}, \rho_{1}\right)$ such that $\pi_{1}$ does not connect $S \triangle T$ to $(S \triangle T)^{c}$ and $\rho_{1}$ does not connect $S$ to $S^{c}$ to any other pair $\left(\pi_{2}, \rho_{2}\right)$ with this property.

We can get from any pairing of nodes in $S$ to any other pairing of nodes in $S$ using moves of type $A_{B W}$ because type $A_{B W}$ moves allow us to exchange any nodes of the same color in $S$. By the same reasoning, we can get from any pairing of nodes in $S^{c}$ to any other pairing of nodes in $S^{c}$. So, if $\rho$ and $\rho^{\prime}$ are two pairings that both do not connect $S$ to $S^{c}$, then we can get from $\rho$ to $\rho^{\prime}$ using a sequence of moves of type $A_{B W}$.

Similarly, we can get from any odd-even pairing of nodes in $S \triangle T$ to any other odd-even pairing of nodes in $S \triangle T$ by swapping nodes of the same parity in $S \triangle T$. We can also get from any odd-even pairing of nodes in $(S \triangle T)^{c}$ to any other odd-even pairing of nodes in $(S \triangle T)^{c}$. So, if $\pi$ and $\pi^{\prime}$ are two odd-even pairings that both do not connect $S \triangle T$ to $(S \triangle T)^{c}$, then we can get from $\pi$ to $\pi^{\prime}$ using a sequence of moves of type $A_{O E}$.

We have thus shown if we have two pairs of pairings $\left(\pi_{1}, \rho_{1}\right)$ and $\left(\pi_{2}, \rho_{2}\right)$ such that $\pi_{i}$ is odd-even and does not connect $S \triangle T$ to $(S \triangle T)^{c}$ and $\rho_{i}$ is black-white and does not connect $S$ to $S^{c}$, that the right hand side of equation (2.6.1) is unchanged when we replace $\left(\pi_{1}, \rho_{1}\right)$ with $\left(\pi_{2}, \rho_{2}\right)$.

2.6.2. Proof that equation (2.6.1) holds. First assume that $S$ is a balanced set of size $2 j$ such that there is a planar black-white pairing $\rho$ that does not connect $S$ to $S^{c}$. Although it may not be obvious that such a set always exists, recall from Lemma 2.1.14 that regardless of the node coloring of $\mathbf{N}$, there exists a planar black-white pairing $\rho$ of $\mathbf{N}$. So we choose $S$ to be $2 j$ of the arcs of $\rho$.

Then by definition,

$$
\operatorname{sign}(S)=(-1)^{\# \text { crosses of } \rho}=1 \text {. }
$$

Let $\pi=\rho$. Since $\pi$ is odd-even and black-white, for all pairs in $\pi$, either both nodes of the pair are in $T$ or both are not in $T$, so $\pi$ does not connect $S \triangle T$ to $(S \triangle T)^{c}$. Since $\pi=\rho,(-1)^{\# \text { nodes } / 2}=$

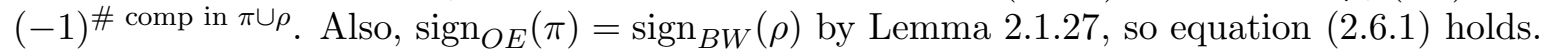


We can obtain any balanced set of size $2 j$ from $S$ by making a sequence of the following types of replacements:

(1) Replace $x \in S$ with $x+1 \in S^{c}$, where $(x, x+1)$ is a couple of consecutive nodes of the same color. (Or replace $x+1 \in S$ with $x \in S^{c}$ ).

(2) Replace $x \in S$ with $y \in S^{c}$, where $x<y$ are the same color and all $\ell$ nodes in the interval $[x+1, x+2, \ldots, y-1]$ are the opposite color of $x$ and $y(\ell \geq 1)$. (Or replace $y \in S$ with $\left.x \in S^{c}\right)$.

Therefore it suffices to show the following. Assume we're given a balanced set $S$, an odd-even pairing $\pi$ that does not connect $S \triangle T$ to $(S \triangle T)^{c}$, and a black-white pairing $\rho$ that does not connect $S$ to $S^{c}$ such that $\left.\rho\right|_{S}$ and $\left.\rho\right|_{S^{c}}$ are planar. After making either of the above two types of replacements to obtain $S^{\prime}$, we can construct an odd-even pairing $\pi^{\prime}$ that does not connect $S^{\prime} \triangle T$ to $\left(S^{\prime} \triangle T\right)^{c}$ and a black-white pairing $\rho^{\prime}$ that does not connect $S^{\prime}$ to $S^{\prime c}$ such that $\left.\rho^{\prime}\right|_{S^{\prime}}$ and $\left.\rho^{\prime}\right|_{S^{\prime c}}$ are planar. After replacing $S, \pi, \rho$ in equation (2.6.1) with $S^{\prime}, \pi^{\prime}$ and $\rho^{\prime}$, equation (2.6.1) still holds.

This requires several lemmas.

Lemma 2.6.8. Let $S$ be a balanced subset of nodes. Let $x$ and $y$ be two nodes of the same color and opposite parity with $x<y$ such that $x \in S$ and $y \notin S$. Let $\rho$ be a black-white pairing such that $\rho$ does not connect $S$ to $S^{c}$ and let $\pi$ be an odd-even pairing such that $\pi$ does not connect $S \triangle T$ to $(S \triangle T)^{c}$. Let $S^{\prime}=S \backslash\{x\} \cup\{y\}$ and let $\rho^{\prime}$ be the pairing obtained by swapping the locations of $x$ and $y$ in $\rho$. Then

(a) if $\pi(x)=y$,

(i) $\pi$ does not connect $S^{\prime} \triangle T$ to $\left(S^{\prime} \triangle T\right)^{c}$, and

(ii) when $\rho$ is replaced with $\rho^{\prime}$, the right hand side of equation (2.6.1) changes sign.

(b) if $\pi(x) \neq y$, let $\pi^{\prime}$ be the pairing obtained from $\pi$ by pairing $x$ with $y, \pi(x)$ with $\pi(y)$, and leaving the remaining pairs the same. Then

(i) $\pi^{\prime}$ does not connect $S^{\prime} \triangle T$ to $\left(S^{\prime} \triangle T\right)^{c}$.

(ii) when $\rho$ is replaced with $\rho^{\prime}$ and $\pi$ is replaced with $\pi^{\prime}$, the right hand side of equation (2.6.1) changes sign.

Proof. We will first prove part (a). The fact that $\pi$ does not connect $S^{\prime} \triangle T$ to $\left(S^{\prime} \triangle T\right)^{c}$ follows from the observation that since $\pi(x)=y$, both $x$ and $y$ are in $S \triangle T$ or both are in $(S \triangle T)^{c}$. If both $x, y$ are in $S \triangle T$ then since we assumed $x \in S$ and $y \notin S, y$ must be in $T$, so both $x, y$ are in $\left(S^{\prime} \triangle T\right)^{c}$. So $\pi$ does not connect $S^{\prime} \triangle T$ to $\left(S^{\prime} \triangle T\right)^{c}$.

Since we obtained $\rho^{\prime}$ from $\rho$ by swapping the locations of $x$ and $y, \operatorname{sign}_{B W}\left(\rho^{\prime}\right)=-\operatorname{sign}_{B W}(\rho)$. The number of components in $\pi \cup \rho$ is the same as the number of components in $\pi \cup \rho^{\prime}$ because when we replace $\rho$ with $\rho^{\prime}$ the path $\pi(\rho(x))-\rho(x)-x-y-\rho(y)$ is replaced with $\pi(\rho(x))-\rho(x)-y-x-\rho(y)$. So the right hand side of equation (2.6.1) changes sign.

Next, we prove part (b). The proof of (i) relies on the observation that since $x$ and $y$ are the same color but opposite parity, exactly one of the nodes $x, y$ is in $T$. This implies that $x$ and $y$ are both in $S \triangle T$ or both in $(S \triangle T)^{c}$ and that $x$ and $y$ are both in $S^{\prime} \triangle T$ or both in $\left(S^{\prime} \triangle T\right)^{c}$.

Since $x$ and $y$ are both in $S \triangle T$ or both in $(S \triangle T)^{c}, \pi(x)$ and $\pi(y)$ are both in $S \triangle T$ or both in $(S \triangle T)^{c}$. Since neither $\pi(x)$ nor $\pi(y)$ is $x$ or $y, \pi(x)$ and $\pi(y)$ are both in $S^{\prime} \triangle T$ or both in $\left(S^{\prime} \triangle T\right)^{c}$. We conclude that $\pi^{\prime}$ does not connect $S^{\prime} \triangle T$ to $\left(S^{\prime} \triangle T\right)^{c}$.

For the proof of (ii), first note that pairing $x$ and $y$ and $\pi(x)$ with $\pi(y)$ is the same as swapping

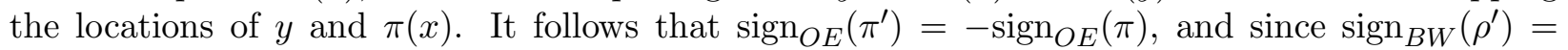
$-\operatorname{sign}_{B W}(\rho)$, it remains to show that the number of components in $\pi^{\prime} \cup \rho^{\prime}$ and the number of components in $\pi \cup \rho$ differ by 1 .

By letting $a=\pi(x)$ and $b=y$ in Definition 2.6.3, we see that $\left(\pi^{\prime}, \rho\right)$ and $(\pi, \rho)$ differ by a move of type $A_{O E}$. If $\pi(x)$ and $y$ are in different components, this is clear, since $\pi(x)$ and $y$ have the same parity. If $\pi(x)$ and $y$ are in the same component, we must show that they are the same color if and only if there are an even number of transition pairs between them. This is because 
- $y$ and $x$ are the same color

- a path from $y$ to $x$ contains an odd number of transition pairs (since $x \in S$ and $y \notin S$ )

- $x$ and $\pi(x)$ are the same color if and only if $(x, \pi(x))$ is a transition pair

So, by Lemma 2.6.5, the number of components in $\pi^{\prime} \cup \rho$ and the number of components in $\pi \cup \rho$ differ by one. Then, since $\pi^{\prime}(x)=y$, by the proof of part (a), the number of components in $\pi^{\prime} \cup \rho^{\prime}$ is the same as the number of components in $\pi^{\prime} \cup \rho$.

We conclude that when $\rho$ is replaced with $\rho^{\prime}$ and $\pi$ is replaced with $\pi^{\prime}$ the right hand side of equation (2.6.1) changes sign.

Lemma 2.6.9. Let $S \subseteq \mathbf{N}$ be a balanced set. Let $x, y$ be nodes of the same color such that $x \in S$, $y \in S^{c}, x<y$ and all $\ell$ nodes in the interval $[x+1, x+2, \ldots, y-1]$ are the opposite color of $x$ and $y(\ell \geq 1)$.

Let $\rho$ be a black-white pairing such that $\rho$ does not connect $S$ to $S^{c}$ and $\left.\rho\right|_{S}$ and $\left.\rho\right|_{S^{c}}$ are planar.

(1) If $\rho(x)$ is not in the interval $[x+1, \ldots, y-1]$ and there is a node in this interval that is in $S$, let $k$ be the smallest integer such that $x+k$ is in $S$ and let $\rho^{\prime}$ be the pairing obtained from $\rho$ by replacing the pairs $(x, \rho(x))$ and $(x+k, \rho(x+k))$ with the pairs $(x, x+k)$ and $(\rho(x), \rho(x+k))$. Then $\left.\rho^{\prime}\right|_{S}$ and $\left.\rho^{\prime}\right|_{S^{c}}$ are planar. Also, replacing $\rho$ with $\rho^{\prime}$ does not change the right hand side of equation (2.6.1).

(2) If $\rho(y)$ is not in the interval $[x+1, \ldots, y-1]$ and there is a node in this interval that is in $S^{c}$, let $k$ be the smallest integer such that $y-k$ is in $S^{c}$ and let $\rho^{\prime}$ be the pairing obtained from $\rho$ by replacing the pairs $(y, \rho(y))$ and $(y-k, \rho(y-k))$ with the pairs $(y, y-k)$ and $(\rho(y), \rho(y-k))$. Then $\left.\rho^{\prime}\right|_{S}$ and $\left.\rho^{\prime}\right|_{S^{c}}$ are planar. Also, replacing $\rho$ with $\rho^{\prime}$ does not change the right hand side of equation (2.6.1).

Proof. Since the proofs of (1) and (2) are completely analogous, we only prove (1).

We first show that $\left.\rho^{\prime}\right|_{S}$ is planar. Since we chose the smallest integer $k$ such that $x+k$ is in $S$, there are no chords connecting two nodes in $S$ that cross the chord $x \frown(x+k)$. We need to check that there are no chords connecting two nodes in $S$ that cross the chord $\rho(x) \frown \rho(x+k)$. If there was such a crossing, that means that there is a node $a \in S$ such that one of the following holds:

(1) $a<\rho(x+k)<\rho(a)<\rho(x)$,

(2) $\rho(x+k)<a<\rho(x)<\rho(a)$,

(3) $a<\rho(x)<\rho(a)<\rho(x+k)$, or

(4) $\rho(x)<a<\rho(x+k)<\rho(a)$.

We use the facts that if $a>x$ then $a>x+k$ (since otherwise $a \in S^{c}$, a contradiction) or, similarly, if $\rho(a)>x$ then $\rho(a)>x+k$, to show that if the inequalities in (1), (2), (3), or (4) hold, then $\left.\rho\right|_{S}$ is not planar.

For example, in case (1), if $a>x$ then $a>x+k$. So we have

$$
x+k<a<\rho(x+k)<\rho(a),
$$

which contradicts that $\left.\rho\right|_{S}$ is planar. If $a<x$ then there are two cases. If $\rho(a)<x$, we have $a<\rho(x+k)<\rho(a)<x+k$. If instead $\rho(a)>x$, we have $a<x<\rho(a)<\rho(x)$. In both cases, we have a contradiction.

In case (2), if $a>x$, then we have $x<a<\rho(x)<\rho(a)$. If $a<x$ and $\rho(a)<x$, then $a<\rho(x)<\rho(a)<x$. If $a<x$ and $\rho(a)>x$, then $\rho(x+k)<a<x+k<\rho(a)$. In all cases, we have a contradiction.

Case (3) is similar to case (2), and case (4) is similar to case (1).

We conclude that $\left.\rho^{\prime}\right|_{S}$ is planar. Since $\left.\rho\right|_{S^{c}}$ was planar and the nodes $x, x+k, \rho(x), \rho(x+k)$ are all in $S,\left.\rho^{\prime}\right|_{S^{c}}$ is also planar.

Next, we observe that the number of components in $\pi \cup \rho$ and the number of components in $\pi \cup \rho^{\prime}$ differ by 1 . This is because to obtain the pairing $\rho^{\prime}$ from $\rho$, we swapped the locations of $x$ and $\rho(x+k)$. Since $x$ and $\rho(x+k)$ are both in $S$ and both the same color, $\left(\pi, \rho^{\prime}\right)$ and $(\pi, \rho)$ differ 
by a move of type $A_{B W}$. So by Corollary 2.6.7, the number of components in $\pi \cup \rho$ and the number of components in $\pi \cup \rho^{\prime}$ differ by 1 . Since $\operatorname{sign}\left(\rho^{\prime}\right)=-\operatorname{sign}(\rho)$, replacing $\rho$ with $\rho^{\prime}$ does not change the right hand side of equation (2.6.1).

The following useful observation is immediate from the definitions.

Remark 2.6.10. Let $\sigma$ be a pairing such that $x$ and $y$ are two nodes that are not paired in $\sigma$, and let $\sigma^{\prime}$ be the pairing obtained by swapping the locations of $x$ and $y$ in $\sigma$. Suppose $S$ is a balanced subset of nodes such that $x \in S$ and $y \in S^{c}$. Let $S^{\prime}=(S \backslash\{x\}) \cup\{y\}$. If $\sigma$ does not connect $S$ to $S^{c}$, then $\sigma^{\prime}$ does not connect $S^{\prime}$ to $S^{\prime c}$.

Proof that equation (2.6.1) holds. Throughout this proof, we assume that we are given a balanced set $S$, an odd-even pairing $\pi$ that does not connect $S \triangle T$ to $(S \triangle T)^{c}$, and a black-white pairing $\rho$ that does not connect $S$ to $S^{c}$ and is planar when restricted to $S$ and when restricted to $S^{c}$.

Recall from the beginning of Section 2.6.2 that we are considering two types of replacements that we can make to $S$ to obtain $S^{\prime}$ : (1) replacing $x \in S$ with $x+1 \in S^{c}$, where $(x, x+1)$ is a couple of consecutive nodes of the same color, and (2) replacing $x \in S$ with $y \in S^{c}$, where $x<y$ are the same color and all $\ell$ nodes appearing between $x$ and $y$ are the opposite color of $x$ and $y$ for some $\ell \geq 1$. For both types of replacements, we will construct a black-white pairing $\rho^{\prime}$ that does not connect $S^{\prime}$ to $S^{\prime c}$ such that $\left.\rho\right|_{S^{\prime}}$ and $\left.\rho\right|_{S^{\prime c}}$ are planar and an odd-even pairing $\pi^{\prime}$ that does not connect $S^{\prime} \triangle T$ to $\left(S^{\prime} \triangle T\right)^{c}$. We will show that after replacing $S, \pi, \rho$ in equation (2.6.1) with $S^{\prime}$, $\pi^{\prime}$ and $\rho^{\prime}$, equation (2.6.1) still holds.

(1) Replace $x \in S$ with $x+1 \in S^{c}$.

Suppose we replace $x \in S$ with $x+1 \in S^{c}$ to obtain $S^{\prime}$. There are two cases to consider based on whether or not $\pi(x)=x+1$. In both cases, we let $\rho^{\prime}$ be the pairing obtained by swapping the locations of $x$ and $x+1$ in $\rho$. By Remark 2.6.10, $\rho^{\prime}$ is a black-white pairing that does not connect $S^{\prime}$ to $S^{\prime c}$. Also note that since $\left.\rho\right|_{S}$ and $\left.\rho\right|_{S^{c}}$ are planar, $\left.\rho^{\prime}\right|_{S^{\prime}}$ and $\left.\rho^{\prime}\right|_{S^{\prime c}}$ are planar.

Case 1. If $\pi(x)=x+1, \pi$ does not connect $S^{\prime} \triangle T$ to $\left(S^{\prime} \triangle T\right)^{c}$ and when we replace $\rho$ with $\rho^{\prime}$, the right hand side of equation (2.6.1) changes sign by Lemma 2.6.8. Since we swapped the locations

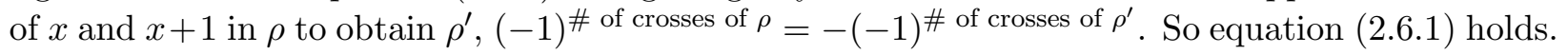

Case 2. If $\pi(x) \neq x+1$, let $\pi^{\prime}$ be the pairing obtained from $\pi$ by pairing $x$ with $x+1$, $\pi(x)$ with $\pi(x+1)$, and leaving the remaining pairs the same. By Lemma 2.6.8, $\pi^{\prime}$ does not connect $S^{\prime} \triangle T$ to $\left(S^{\prime} \triangle T\right)^{c}$ and when we replace $\pi$ with $\pi^{\prime}$ and $\rho$ with $\rho^{\prime}$, the right hand side of equation (2.6.1) changes sign. As in Case 1, $(-1)^{\#}$ of crosses of $\rho=-(-1)^{\#}$ of crosses of $\rho^{\prime}$, so equation (2.6.1) holds.

(2) Replace $x$ with $y$, where $x<y$ are the same color and all $\ell$ nodes in the interval $[x+1, x+2, \ldots, y-1]$ are the opposite color of $x$ and $y(\ell \geq 1)$.

Suppose we replace $x \in S$ with $y \in S^{c}$ to obtain $S^{\prime}$. There are several cases to consider based on whether $x$ and $y$ are paired with nodes in the interval $[x+1, x+2, \ldots, y-1]$.

Case 1. We first consider the case when both $x$ and $y$ are paired with a node in the interval $[x+1, x+2, \ldots, y-1]$.

Construction of $\rho^{\prime}$. Let $\rho^{(1)}$ be the pairing obtained by swapping the locations of $x$ and $y$. By Remark 2.6.10, $\rho^{(1)}$ does not connect $S^{\prime}$ to $S^{\prime c}$. 
We observe that if $\ell>2$, at least one of $\left.\rho^{(1)}\right|_{S^{\prime}},\left.\rho^{(1)}\right|_{S^{\prime c}}$ is not planar. To see this, observe that since $\left.\rho\right|_{S}$ and $\left.\rho\right|_{S^{c}}$ are planar, the nodes in the interval $[x+1, \ldots, \rho(x)-1]$ are in $S^{c}$ and the nodes in the interval $[\rho(y)+1, \ldots, y-1]$ are in $S$ (see Figure 11).

Suppose towards a contradiction that $\left.\rho^{(1)}\right|_{S^{\prime}}$ and $\left.\rho^{(1)}\right|_{S^{\prime c}}$ are planar. Since $\left.\rho^{(1)}\right|_{S^{\prime c}}$ is planar, all nodes in the interval $[x+1, \ldots, \rho(y)-$ 1] are in $S^{\prime}$. This means that either

(1) $\rho(y)=x+1$, or

(2) $\rho(x)=x+1$ and $\rho(y)=x+2$.

If (1) holds, there is at least one node in the interval $[\rho(y)+1, \ldots, y-$ 1] other than $\rho(x)$. By the observation in the previous paragraph, this node is in $S^{\prime}$. If it is in the interval $[\rho(x)+1, \ldots, y-1]$ its chord crosses the $\rho(x) \frown y$ chord, contradicting the assumption that $\left.\rho^{(1)}\right|_{S^{\prime}}$ is planar. If it is in the interval $[x+1, \ldots, \rho(x)-1]$ it crossed the $\rho(x) \frown x$ chord, contradicting the planarity of $\left.\rho\right|_{S}$. If (2) holds, there is at least one node in the interval $[\rho(y)+1, \ldots, y-1]$, this node is in $S^{\prime}$, and its chord crosses the $\rho(x) \frown y$ chord, contradicting the assumption that $\left.\rho^{(1)}\right|_{S^{\prime}}$ is planar.

Observe that since $\rho$ pairs $x$ and $y$ with nodes in the interval $[x+1, x+2, \ldots, y-1]$, any crossings in $\left.\rho^{(1)}\right|_{S^{\prime}}$ must involve nodes in the interval $[x+1, x+2, \ldots, y-1]$.

We claim that we can undo the crossings in $\left.\rho^{(1)}\right|_{S^{\prime}}$ one at a time without changing the right hand side of equation (2.6.1). To prove the claim, we will describe a procedure for constructing $\rho^{(m+1)}$ from $\rho^{(m)}$ so that $\left.\rho^{(m+1)}\right|_{S^{\prime}}$ has one fewer crossing than $\left.\rho^{(m)}\right|_{S^{\prime}}$.

Procedure 2.6.11. (Illustrated in Figure 12). Choose the smallest node $i_{m} \in S^{\prime}$ greater than $\rho^{(m)}(y)$ such that $i_{m} \frown \rho^{(m)}\left(i_{m}\right)$ crosses the chord $y \frown \rho^{(m)}(y)$. Note that $\rho^{(m)}\left(i_{m}\right)=\rho\left(i_{m}\right)$ for all $m$ and $\rho^{(1)}(y)=\rho(x)$. Since $i_{m}$ and $\rho^{(m)}(y)$ are the same color and both in $S^{\prime}$, we can swap the locations of $i_{m}$ and $\rho^{(m)}(y)$ in $\rho^{(m)}$ to obtain $\rho^{(m+1)}$, and this is a move of type $A_{B W}$. By Corollary 2.6.7, replacing $\rho^{(m)}$ with $\rho^{(m+1)}$ does not change the right hand side of equation (2.6.1). We claim that $\left.\rho^{(m+1)}\right|_{S^{\prime}}$ has one fewer crossing than $\left.\rho^{(m)}\right|_{S^{\prime}}$. First observe that since all nodes between $x$ and $y$ are the same color, any chord that crosses the chord $i_{m} \frown y$ must have also crossed the chord $y \frown \rho^{(m)}(y)$. So we just need to check that pairing $\rho^{(m)}(y)$ with $\rho\left(i_{m}\right)$ did not create any crossings. If a black-white chord $a \frown \rho(a)$ with $a, \rho(a) \in S^{\prime}$ crosses $\rho^{(m)}(y) \frown \rho\left(i_{m}\right)$, then if either one of $a, \rho(a)$ is in the interval $\left[\rho^{(m)}(y)+1, \ldots, i_{m}-1\right]$, it would have crossed $y \frown \rho^{(m)}(y)$, contradicting the assumption that $i_{m}$ is the node in $S^{\prime}$ closest to $\rho^{(m)}(y)$ that crossed $y \frown \rho^{(m)}(y)$. So both $a, \rho(a)$ are outside the interval $\left[\rho^{(m)}(y)+1, \ldots, i_{m}-1\right]$, meaning $a \frown \rho(a)$ crosses $\rho^{(m)}(y) \frown \rho\left(i_{m}\right)$ if and only if it crosses $i_{m} \frown \rho\left(i_{m}\right)$.

Note that if $\rho^{(m)}$ does not connect $S^{\prime}$ to $S^{\prime c}$, then $\rho^{(m+1)}$ does not connect $S^{\prime}$ to $S^{\prime c}$. We repeat this procedure until we have a pairing $\rho^{(n)}$ such that $\left.\rho^{(n)}\right|_{S^{\prime}}$ is planar.

Similarly, we can undo the crossings in $\left.\rho^{(n)}\right|_{S^{\prime c}}$ one at a time without changing the right hand side of equation (2.6.1). The resulting pairing is $\rho^{\prime}$.

Construction of $\pi^{\prime}$ and analysis of equation (2.6.1).

We break into subcases based on the parity of $\ell$.

Case 1a. $\ell$ is odd

Analysis of LHS of (2.6.1). Since $x$ and $y$ are both paired with black nodes in the interval $[x+$

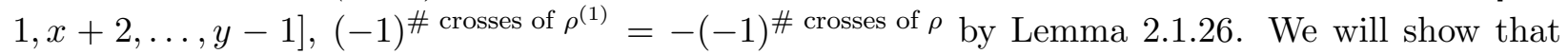
when we undo crossings to obtain $\rho^{\prime}$ as described, we apply Procedure 2.6.11 an odd number of 

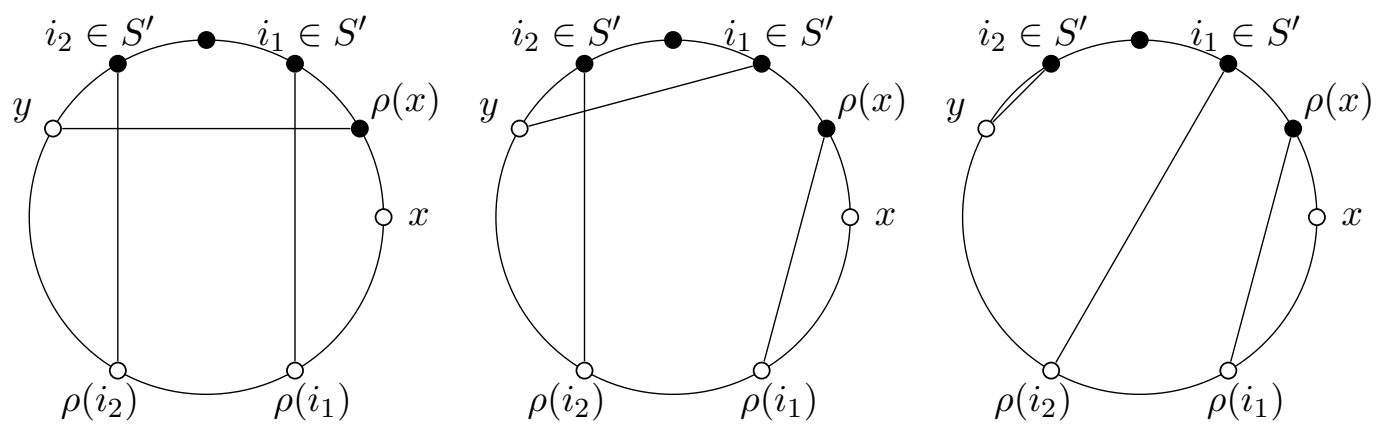

Figure 12. Illustration of the procedure for undoing the crossings in $\left.\rho^{(1)}\right|_{S^{\prime}}$. Left: Choose the smallest node $i_{1}$ in $S^{\prime}$ greater than $\rho^{(1)}(y)=\rho(x)$ whose chord crosses the chord $y \frown \rho(x)$. Center: Swap the locations of $i_{1}$ and $\rho(x)$ in $\rho^{(1)}$ to obtain $\rho^{(2)}$. Right: Repeat this procedure to obtain $\rho^{(2)}$.

times. Recall that every node between $x$ and $\rho(x)$ is in $S^{c}$ and every node between $\rho(y)$ and $y$ is in $S$. It follows that $\rho(x)<\rho(y)$ or $\rho(x)=\rho(y)+1$. Putting these facts together, we see that every node in $S^{\prime c} \cap\{x+1, \ldots, y-1\}$ crosses the $x \frown \rho(y)$ chord, and every node in $S^{\prime} \cap\{x+1, \ldots, y-1\}$ crosses the $y \frown \rho(x)$ chord. Since there are an odd number of nodes in $\{x+1, \ldots, y-1\} \backslash\{\rho(x), \rho(y)\}$, we must apply Procedure 2.6.11 an odd number of times. We

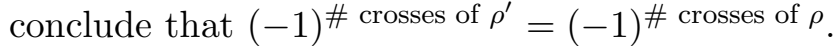

Construction of $\pi^{\prime}$ and analysis of RHS of (2.6.1). Since $\ell$ is odd, $x$ and $y$ are the same parity, so we let $\pi^{\prime}$ be the pairing obtained by swapping the locations of $x$ and $y$. We claim that $\pi^{\prime}$ does not connect $S^{\prime} \triangle T$ to $\left(S^{\prime} \triangle T\right)^{c}$. Since $x$ and $y$ are the same parity and the same color, either both of $x, y$ are in $T$ or neither $x$ nor $y$ are in $T$. Since $x, y$ are either both in $T$ or both not in $T$, exactly one of $x, y$ is in $S \triangle T$. So by Remark 2.6.10, $\pi^{\prime}$ does not connect $S^{\prime} \triangle T$ to $\left(S^{\prime} \triangle T\right)^{c}$.

Also, $\pi^{\prime} \cup \rho^{(1)}$ has the same number of components as $\pi \cup \rho$ because when we replace $\pi$ with $\pi^{\prime}$ and $\rho$ with $\rho^{(1)}$, the path $\cdots-\pi(x)-x-\rho(x)-\cdots$ in $\pi \cup \rho$ is replaced with $\cdots-\pi(y)-x-\rho(y)-\cdots$ in $\pi^{\prime} \cup \rho^{(1)}$ and the path $\cdots-\pi(y)-y-\rho(y)-\cdots$ in $\pi \cup \rho$ is replaced with $\cdots-\pi(x)-y-\rho(x)-\cdots$.

Since we applied Procedure 2.6.11 an odd number of times and each application of Procedure 2.6.11 is a move of type $A_{B W}$, by Lemma 2.6.5,

$$
(-1)^{\# \text { comp in } \pi^{\prime} \cup \rho^{\prime}}=-(-1)^{\# \text { comp in } \pi^{\prime} \cup \rho^{(1)}}=-(-1)^{\# \text { comp in } \pi \cup \rho} \text {. }
$$

Since $\operatorname{sign}_{B W}\left(\rho^{(1)}\right)=-\operatorname{sign}_{B W}(\rho)$ and $\operatorname{sign}_{B W}\left(\rho^{(m+1)}\right)=-\operatorname{sign}_{B W}\left(\rho^{(m)}\right), \operatorname{sign}_{B W}\left(\rho^{\prime}\right)=\operatorname{sign}_{B W}(\rho)$. Finally, since $\operatorname{sign}_{O E}\left(\pi^{\prime}\right)=-\operatorname{sign}_{O E}(\pi)$, we conclude that equation (2.6.1) holds when $\pi$ is replaced with $\pi^{\prime}$ and $\rho$ is replaced with $\rho^{\prime}$.

Case 1b. $\ell$ is even

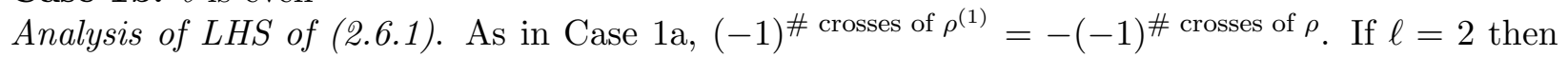
we let $\rho^{\prime}=\rho^{(1)}$ and both $\left.\rho^{\prime}\right|_{S^{\prime}}$ and $\left.\rho^{\prime}\right|_{S^{\prime c}}$ are planar. If $\ell>2$, then we will show that when we undo crossings in $\rho^{(1)}$ to obtain $\rho^{\prime}$ we apply Procedure 2.6.11 an even number of times. The reasoning is analogous to the $\ell$ is odd case: the claim follows from the fact that there are an even number of

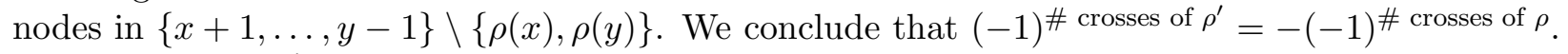
Construction of $\pi^{\prime}$ and analysis of RHS of (2.6.1). We break into cases based on whether $\pi(x)=y$ or $\pi(x) \neq y$. If $\pi(x)=y$, we let $\pi^{\prime}=\pi$. If $\pi(x) \neq y$, we let $\pi^{\prime}$ be the pairing obtained from $\pi$ by pairing $x$ with $y, \pi(x)$ with $\pi(y)$, and leaving the remaining pairs the same. In both cases $\pi^{\prime}$ does not connect $S^{\prime} \triangle T$ to $\left(S^{\prime} \triangle T\right)^{c}$ and $\operatorname{sign}_{O E}(\pi)(-1)^{\# \text { comp in } \pi \cup \rho}=\operatorname{sign}_{O E}\left(\pi^{\prime}\right)(-1)^{\# \text { comp in } \pi^{\prime} \cup \rho^{(1)}}$ by Lemma 2.6.8. Since we applied Procedure 2.6.11 an even number of times and each application of

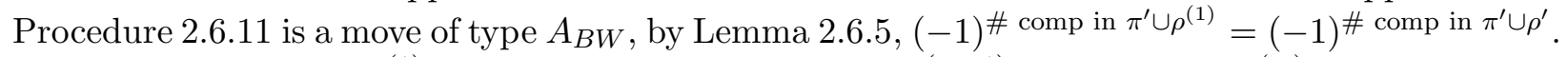
Finally, since $\operatorname{sign}_{B W}\left(\rho^{(1)}\right)=-\operatorname{sign}_{B W}(\rho)$ and $\operatorname{sign}_{B W}\left(\rho^{(m+1)}\right)=-\operatorname{sign}_{B W}\left(\rho^{(m)}\right), \operatorname{sign}_{B W}\left(\rho^{\prime}\right)=$ 
$-\operatorname{sign}_{B W}(\rho)$. We conclude that when $\rho$ is replaced with $\rho^{\prime}$ and $\pi$ is replaced with $\pi^{\prime}$, the right hand side of equation (2.6.1) changes sign. Thus equation (2.6.1) holds.

Case 2. We next consider the case where exactly one of $x$ or $y$ is paired with a black node in the interval $[x+1, x+2, \ldots, y-1]$.

Without loss of generality, suppose that $x$ is the node that is paired with a black node in the interval $[x+1, \ldots, y-1]$. There are two subcases to consider.

Case 2a. If one of the $\ell$ nodes between $x$ and $y$ is in $S^{c}$, then let $k$ be the smallest integer such that $y-k$ is in $S^{c}$ and let $\rho^{\prime}$ be the pairing obtained by pairing $y$ with $y-k$ and $\rho(y)$ with $\rho(y-k)$. By Lemma 2.6.9, $\left.\rho^{\prime}\right|_{S}$ and $\left.\rho^{\prime}\right|_{S^{c}}$ are planar, and replacing $\rho$ with $\rho^{\prime}$ does not change the right hand side of equation (2.6.1).

To show that replacing $\rho$ with $\rho^{\prime}$ does not change the left hand side of equation (2.6.1), we must

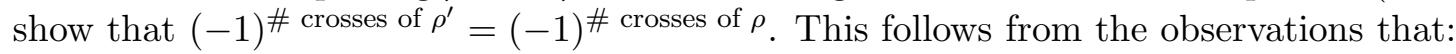

- since $\left.\rho\right|_{S^{c}}$ is planar, the chords $(y-k) \frown \rho(y-k)$ and $y \frown \rho(y)$ do not cross, and

- a chord $a \frown \rho(a)$ crosses exactly one of $(y-k) \frown \rho(y-k), y \frown \rho(y)$ if and only if it crosses exactly one of $\rho(y-k) \frown \rho(y),(y-k) \frown y$.

Thus we have reduced Case 2a to Case 1, where both $x$ and $y$ are paired with nodes in the interval $[x+1, x+2, \ldots, y-1]$.

Case 2b. If all of the $\ell$ nodes between $x$ and $y$ are in $S$ (this includes the case where the only node between $x$ and $y$ is $x+1$ ), then since $\left.\rho\right|_{S}$ is planar, $x$ is paired with $x+1$. When we swap the locations of $x$ and $y$ to obtain $\rho^{(1)},\left.\rho^{(1)}\right|_{S^{\prime c}}$ is planar but $\left.\rho^{(1)}\right|_{S^{\prime}}$ is not planar. In fact, every node between $x+1$ and $y$ is in $S$ (and therefore in $S^{\prime}$ ) and crosses the $y \frown(x+1)$ chord. As in Case 1, we obtain $\rho^{\prime}$ by applying Procedure 2.6.11 to undo the crossings in $\left.\rho^{(1)}\right|_{S^{\prime}}$, and this does not change the right hand side of equation (2.6.1). We break into cases based on whether $\ell$ is odd or $\ell$ is even before constructing $\pi^{\prime}$.

Case 2bi. $\ell$ is odd

Since exactly one of $x$ and $y$ is paired with a node in the interval $[x+1, x+2, \ldots, y-1]$,

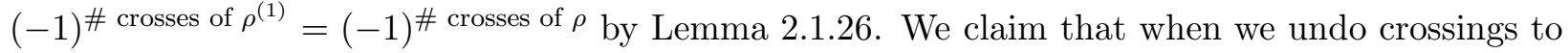
obtain $\rho^{\prime}$, there are an even number of crossings to undo. This is because every node between $x+1$ and $y$ crosses the $(x+1) \frown y$ chord, and since $\ell$ is odd there are an even number of such nodes. So

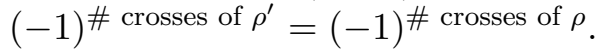

We let $\pi^{\prime}$ be the pairing obtained by swapping the locations of $x$ and $y$. By the type of arguments

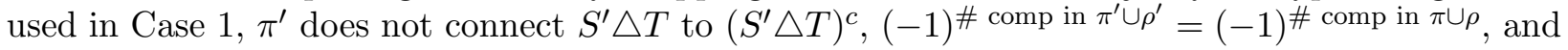
$\operatorname{sign}_{B W}\left(\rho^{\prime}\right)=-\operatorname{sign}_{B W}(\rho)$. We conclude that equation (2.6.1) holds when $\pi$ is replaced with $\pi^{\prime}$ and $\rho$ is replaced with $\rho^{\prime}$.

Case 2bii. $\ell$ is even

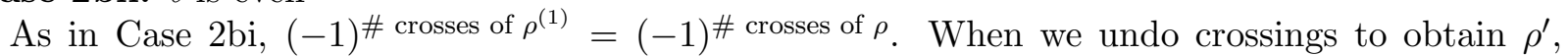

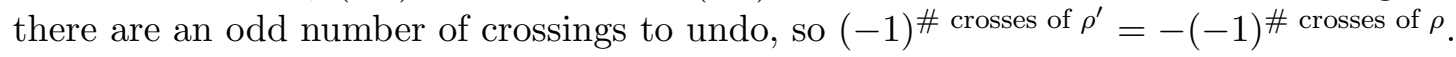

We break into cases based on whether $\pi(x)=y$ or $\pi(x) \neq y$. If $\pi(x)=y$, we let $\pi^{\prime}=\pi$. If $\pi(x) \neq y$, we let $\pi^{\prime}$ be the pairing obtained from $\pi$ by pairing $x$ with $y, \pi(x)$ with $\pi(y)$, and leaving the remaining pairs the same. In both cases $\pi^{\prime}$ does not connect $S^{\prime} \triangle T$ to $\left(S^{\prime} \triangle T\right)^{c}$, and

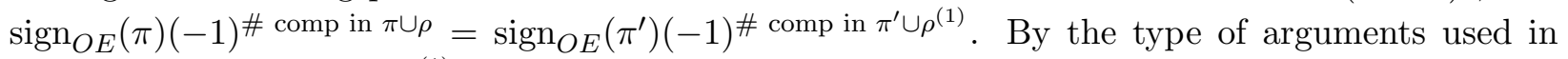

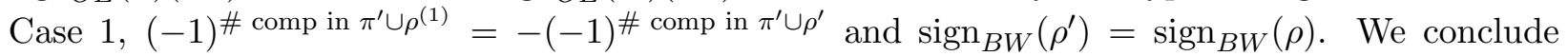
that when when $\rho$ is replaced with $\rho^{\prime}$ and $\pi$ is replaced with $\pi^{\prime}$, the right hand side of equation (2.6.1) changes sign. Thus equation (2.6.1) holds.

Case 3. Finally, we observe that we can reduce the case where neither $x$ nor $y$ is paired with a black node in the interval $[x+1, x+2, \ldots, y-1]$ to the case where exactly one of $x$ or $y$ is paired with a black node in the interval $[x+1, x+2, \ldots, y-1]$. 
First assume that at least one of the $\ell$ nodes between $x$ and $y$ is in $S$. Choose the smallest integer $k$ such that $x+k$ is in $S$. Let $\rho^{\prime}$ be the pairing that pairs $x$ with $x+k$ and $\rho(x)$ with $\rho(x+k)$. By Lemma 2.6.9, $\left.\rho^{\prime}\right|_{S}$ is planar and $\left.\rho\right|_{S^{c}}$ are planar and replacing $\rho$ to $\rho^{\prime}$ does not change the right

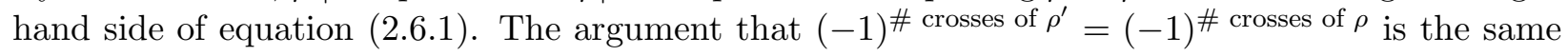
as the argument in Case 2a.

Finally, if all of the $\ell$ nodes between $x$ and $y$ are in $S^{c}$, pair $y$ with $x+\ell$. The argument then proceeds identically.

2.6.3. Proof that $(\pi, \rho)$ exists. We conclude by proving the existence of an odd-even pairing $\pi$ and a black-white pairing $\rho$ such that $\pi$ does not connect $S \triangle T$ to $(S \triangle T)^{c}$ and $\rho$ does not connect $S$ to $S^{c}$.

Recall that at the beginning of Section 2.6.2 we showed that for all $j$ there is a balanced set $S$ of size $2 j$ with a planar black-white pairing $\rho$ that does not connect $S$ to $S^{c}$, and by choosing $\pi=\rho$ we also have an odd-even pairing $\pi$ that does not connect $S \triangle T$ to $(S \triangle T)^{c}$.

We also showed that any balanced set of size $2 j$ can be obtained from $S$ by making a sequence of replacements of types (1) and (2) discussed in the beginning of Section 2.6.2. Furthermore, we showed that given an odd-even pairing $\pi$ and a black-white pairing $\rho$ such that $\pi$ does not connect $S \triangle T$ to $(S \triangle T)^{c}$ and $\rho$ does not connect $S$ to $S^{c}$, and a set $S^{\prime}$ obtained from $S$ by making a replacement of the form (1) or (2), we can modify $\pi$ and $\rho$ to obtain $\pi^{\prime}$ and $\rho^{\prime}$ so that $\pi^{\prime}$ does not connect $S^{\prime} \triangle T$ to $\left(S^{\prime} \triangle T\right)^{c}$ and $\rho^{\prime}$ does not connect $S^{\prime}$ to $S^{\prime c}$.

We conclude that for each balanced subset $S$, there is an odd-even pairing $\pi$ and a black-white pairing $\rho$ with the desired properties.

\section{A RECURRENCE FOR TRIPARTITE DOUBLE-DIMER CONFIGURATIONS}

3.1. Kenyon and Wilson's determinant formula. In this section we prove our analogue of Kenyon and Wilson's determinant formula for tripartite pairings. Recall the statement of their theorem from Section 1.2.1:

Theorem 1.2.1. [KW09, Theorem 6.1] Suppose that the nodes are contiguously colored red, green, and blue (a color may occur zero times), and that $\sigma$ is the (unique) planar pairing in which like colors are not paired together. We have

$$
\widehat{\operatorname{Pr}}(\sigma)=\operatorname{sign}_{O E}(\sigma) \operatorname{det}\left[1_{i, j} \text { RGB-colored differently } X_{i, j}\right]_{j=2,4 \ldots, 2 n}^{i=1,3, \ldots, 2 n-1} .
$$

Kenyon and Wilson proved Theorem 1.2.1 by combining two key results. The first is from their study of groves (see Section 2.4). Recall that Kenyon and Wilson showed in Theorem 2.4.5 that $\dddot{\operatorname{Pr}}(\sigma)$ is an integer-coefficient homogeneous polynomial in the variables $L_{i, j}$. Furthermore, they showed that when $\sigma$ is a partition that is a tripartite pairing, the grove polynomial $\dddot{\operatorname{Pr}}(\sigma)$ can be expressed as a Pfaffian whose entries are $L_{i, j}$ or 0 .

Theorem 3.1.1. [KW09, Theorem 3.1] Let $\sigma$ be the tripartite pairing partition defined by circularly contiguous sets of nodes $R, G$, and $B$, where $|R|,|G|$, and $|B|$ satisfy the triangle inequality. Then

$$
\dddot{\operatorname{Pr}}(\sigma)=\operatorname{Pf}\left(\begin{array}{ccc}
0 & L_{R, G} & L_{R, B} \\
-L_{G, R} & 0 & L_{G, B} \\
-L_{B, R} & -L_{B, G} & 0
\end{array}\right)
$$

where $L$ is the matrix with entries $L_{i, j}$ whose rows and columns are indexed by the nodes, and $L_{R, G}$ is the submatrix of $L$ whose rows are the red nodes and columns are the green nodes.

The second result they needed is a theorem which allows one to compute the double-dimer polynomials $\widehat{\operatorname{Pr}}(\sigma)$ using the grove polynomials. 
Theorem 3.1.2. [KW11a, Theorem 4.2] If a planar partition $\sigma$ only contains pairs and we make the following substitutions to the grove partition polynomial $\dddot{\operatorname{Pr}}(\sigma)$ :

$$
L_{i, j} \rightarrow \begin{cases}0, & \text { if } i \text { and } j \text { have the same parity } \\ (-1)^{(|i-j|-1) / 2} X_{i, j}, & \text { otherwise }\end{cases}
$$

then the result is $\operatorname{sign}_{O E}(\sigma)$ times the double-dimer pairing polynomial $\widehat{\operatorname{Pr}}(\sigma)$, when we interpret $\sigma$ as a pairing.

We prove Theorem 1.3.2 (our version of Theorem 1.2.1) similarly. We can use Theorem 3.1 .1 as stated, but we need the following analogue of Theorem 3.1.2:

Theorem 3.1.3. If a planar partition $\sigma$ only contains pairs and we make the following substitutions to the grove partition polynomial $\dddot{\operatorname{Pr}}(\sigma)$ :

$$
L_{i, j} \rightarrow \begin{cases}0, & \text { if } i \text { and } j \text { are the same color } \\ \operatorname{sign}(i, j) Y_{i, j}, & \text { otherwise }\end{cases}
$$

then the result is $\operatorname{sign}_{c}(\mathbf{N}) \operatorname{sign}_{O E}(\sigma) \widetilde{\operatorname{Pr}}(\sigma)$.

Proof. In Theorem 1.3.1, we established that

$$
\widetilde{\operatorname{Pr}}(\sigma):=\frac{Z_{\sigma}^{D D}(G, \mathbf{N})}{\left(Z^{D}(G)\right)^{2}}=\sum_{\text {black-white pairings } \rho} \mathcal{Q}_{\sigma, \rho}^{(D D)} Y_{\rho}^{\prime} .
$$

In the proof of Theorem 1.3.1, we showed $\mathcal{Q}_{\sigma, \rho}^{(D D)}=\operatorname{sign}_{O E}(\sigma) \operatorname{sign}_{B W}(\rho) \mathcal{P}_{\sigma, \rho}^{(t)}$ (see equation (2.5.5)). This connects the polynomials $\widetilde{\operatorname{Pr}}(\sigma)$ to the grove polynomials $\dddot{\operatorname{Pr}}(\sigma)$, since $\dddot{\operatorname{Pr}}(\sigma)=\sum_{\text {partitions } \tau} \mathcal{P}_{\sigma, \tau}^{(t)} L_{\tau}$ by Theorem 2.4.5.

Specifically, in the case where $\sigma$ is a pair, we have

$$
\dddot{\operatorname{Pr}}(\sigma)=\operatorname{sign}_{O E}(\sigma) \sum_{\text {pairs } \rho} \operatorname{sign}_{B W}(\rho) \mathcal{Q}_{\sigma, \rho}^{(D D)} L_{\rho} .
$$

Observe the sum is over all pairs $\rho$ rather than all partitions. This is because by Rule 2.4.4, when we express a partition as a linear combination of planar partitions, any singleton parts of that partition show up in each planar partition with nonzero coefficient. Also observe that when we apply Rule 2.4.4 to a partition, each of the resulting partitions contains the same number of parts as the original partition. It follows that if $\sigma$ is a pairing, and $\mathcal{P}_{\sigma, \rho}^{(t)} \neq 0$ for some partition $\rho$, then $\rho$ is also pairing.

Finally, we recall that $Y_{\rho}^{\prime}=(-1)^{\# \text { crosses of } \rho} \prod_{i \text { black }} Y_{i, \rho(i)}$ and by Lemma 2.1.13,

$$
\operatorname{sign}_{\mathrm{c}}(\mathbf{N}) \operatorname{sign}_{B W}(\rho) \prod_{(i, j) \in \rho} \operatorname{sign}(i, j)=(-1)^{\# \text { crosses of } \rho} .
$$

The theorem follows.

The remainder of this section will be devoted to proving the following theorem.

Theorem 1.3.2. Suppose that the nodes are contiguously colored red, green, and blue (a color may occur zero times), and that $\sigma$ is the (unique) planar pairing in which like colors are not paired together. We have

$$
\widetilde{\operatorname{Pr}}(\sigma)=\operatorname{sign}_{O E}(\sigma) \operatorname{det}\left[1_{i, j} \text { RGB-colored differently } Y_{i, j}\right]_{j=w_{1}, w_{2}, \ldots, w_{n}}^{i=b_{1}, b_{2}, \ldots, b_{n}} .
$$

where $b_{1}<b_{2}<\ldots<b_{n}$ are the black nodes listed in increasing order and $w_{1}<w_{2}<\ldots<w_{n}$ are the white nodes listed in increasing order. 
While our proof of Theorem 1.3.2 is very similar to Kenyon and Wilson's proof of Theorem 1.2.1, we do require the following technical lemma.

Lemma 3.1.4. Let $\mathbf{N}$ be a set of $2 n$ nodes and let $\left(n_{1}, n_{1}+1\right), \ldots,\left(n_{2 k}, n_{2 k}+1\right)$ be a complete list of couples of consecutive nodes of the same color. Define $(-1)^{i>j}$ to be -1 if $i>j$, and 1 otherwise, and let

$$
M=\left[(-1)^{i>j} \operatorname{sign}(i, j) Y_{i, j}\right]_{j=w_{1}, w_{2}, \ldots, w_{n}}^{i=b_{1}, b_{2}, \ldots, b_{n}},
$$

where $b_{1}<b_{2}<\cdots<b_{n}$ are the black nodes listed in increasing order and $w_{1}<w_{2}<\cdots<w_{n}$ are the white nodes listed in increasing order. Then $M$ is a block matrix where within each block, the signs of the entries are staggered in a checkerboard pattern.

Furthermore, let $t$ be the total number of rows and columns of $M$ that we need to multiply by -1 to obtain a matrix with entries whose signs are staggered in a checkerboard pattern where the upper left entry is positive. If node 1 is black,

$$
(-1)^{t}=\operatorname{sign}_{c}(\mathbf{N})(-1)^{\sum_{i=1}^{2 k}\left\lfloor\frac{n_{i}}{2}\right\rfloor}
$$

and if node 1 is white,

$$
(-1)^{t}=(-1)^{n} \operatorname{sign}_{c}(\mathbf{N})(-1)^{\sum_{i=1}^{2 k}\left\lfloor\frac{n_{i}}{2}\right\rfloor} .
$$

Proof. We will first prove the claim that $M$ is a block matrix where within each block, the signs of the entries are staggered in a checkerboard pattern.

We begin with an example. Suppose we have 20 nodes colored as shown right. Then there are four couples of consecutive nodes of the same color: $(4,5),(8,9),(13,14)$, and $(17,18)$ and $M$ is the matrix shown below. We see that the blocks of $M$ correspond to consecutive nodes of the same color. More precisely, the last column in a block corresponds to a white node that precedes at least two consecutive black nodes. The first column in the next block corresponds to the first white node after these consecutive black nodes. Similarly, the nodes corresponding to the last row in a block and the first row in the next block are separated by at least two consecutive

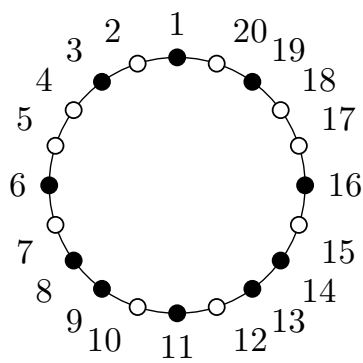
white nodes.

$$
\left(\begin{array}{cccc|cc|cccc}
Y_{1,2} & -Y_{1,4} & Y_{1,5} & -Y_{1,7} & -Y_{1,10} & Y_{1,12} & Y_{1,15} & -Y_{1,17} & Y_{1,18} & -Y_{1,20} \\
-Y_{3,2} & Y_{3,4} & -Y_{3,5} & Y_{3,7} & Y_{3,10} & -Y_{3,12} & -Y_{3,15} & Y_{3,17} & -Y_{3,18} & Y_{3,20} \\
\hline-Y_{6,2} & Y_{6,4} & -Y_{6,5} & Y_{6,7} & Y_{6,10} & -Y_{6,12} & -Y_{6,15} & Y_{6,17} & -Y_{6,18} & Y_{6,20} \\
Y_{8,2} & -Y_{8,4} & Y_{8,5} & -Y_{8,7} & -Y_{8,10} & Y_{8,12} & Y_{8,15} & -Y_{8,17} & Y_{8,18} & -Y_{8,20} \\
-Y_{9,2} & Y_{9,4} & -Y_{9,5} & Y_{9,7} & Y_{9,10} & -Y_{9,12} & -Y_{9,15} & Y_{9,17} & -Y_{9,18} & Y_{9,20} \\
Y_{11,2} & -Y_{11,4} & Y_{11,5} & -Y_{11,7} & -Y_{11,10} & Y_{11,12} & Y_{11,15} & -Y_{11,17} & Y_{11,18} & -Y_{11,20} \\
-Y_{13,2} & Y_{13,4} & -Y_{13,5} & Y_{13,7} & Y_{13,10} & -Y_{13,12} & -Y_{13,15} & Y_{13,17} & -Y_{13,18} & Y_{13,20} \\
Y_{14,2} & -Y_{14,4} & Y_{14,5} & -Y_{14,7} & -Y_{14,10} & Y_{14,12} & Y_{14,15} & -Y_{14,17} & Y_{14,18} & -Y_{14,20} \\
-Y_{16,2} & Y_{16,4} & -Y_{16,5} & Y_{16,7} & Y_{16,10} & -Y_{16,12} & -Y_{16,15} & Y_{16,17} & -Y_{16,18} & Y_{16,20} \\
\hline-Y_{19,2} & Y_{19,4} & -Y_{19,5} & Y_{19,7} & Y_{19,10} & -Y_{19,12} & -Y_{19,15} & Y_{19,17} & -Y_{19,18} & Y_{19,20}
\end{array}\right) .
$$

Since in the matrix above, row $i$ does not correspond to node $i$, we introduce the following notation. We define the map $B:\{1,2, \ldots, n\} \rightarrow\left\{b_{1}, \ldots, b_{n}\right\}$ by letting $B(i)$ be the node corresponding to row $i$. Similarly, we define $W:\{1,2, \ldots, n\} \rightarrow\left\{w_{1}, \ldots, w_{n}\right\}$ by letting $W(j)$ be the node corresponding to column $j$. In the example above, $B(4)=8$ and $W(8)=17$. 
We will show that $M$ has the form

\begin{tabular}{|c|c|c|c|c|}
\hline & $W(j)<s_{1}$ & $s_{1}<W(j)<s_{2}$ & $\cdots$ & $s_{k-1}<W(j)<s_{k}$ \\
\hline$B(i)<u_{1}$ & $A_{1,1}$ & $A_{1,2}$ & $\cdots$ & $A_{1, k}$ \\
\hline$u_{1}<B(i)<u_{2}$ & $A_{2,1}$ & $A_{2,2}$ & $\ldots$ & $A_{2, k}$ \\
\hline$\vdots$ & : & . & $\ddots$ & $\vdots$ \\
\hline$k-1<B(i)<u_{k}$ & $A_{k, 1}$ & $A_{k, 2}$ & $\cdots$ & $A_{k, k}$ \\
\hline
\end{tabular}

where in each block $A_{i, j}$, the signs of the entries are staggered in a checkerboard pattern. Note that a block could be empty.

We first show that within a block, rows $i$ and $i+1$ have opposite sign. There are two cases to consider:

(1) $B(i+1)-B(i)=2$, and

(2) $B(i+1)-B(i)=1$.

These are the only cases because if $B(i+1)-B(i)>2$, then there is at least one couple of consecutive white nodes between $B(i)$ and $B(i+1)$, so rows $i$ and $i+1$ are in different blocks.

In case (1), there is not a couple of consecutive nodes of the same color between $B(i)$ and $B(i+1)$, so $a_{B(i), w}=a_{B(i+1), w}$ for all $w$. It follows immediately from the definition $\operatorname{sign}(b, w)=$ $(-1) \frac{|b-w|+a_{b, w}-1}{2}$ that $\operatorname{sign}(B(i+1), w)=-\operatorname{sign}(B(i), w)$ unless $B(i)<w<B(i+1)$. But if $B(i)<w<B(i+1)$, the sign $(-1)^{b>w}$ flips. So in case (1), rows $i$ and $i+1$ have opposite sign.

In case $(2),(B(i), B(i+1))$ is a couple of consecutive black nodes, so $\left|a_{B(i+1), w}-a_{B(i), w}\right|=1$. If $B(i+1)>w$,

$$
\operatorname{sign}(B(i+1), w)=(-1) \frac{B(i+1)-w+a_{B(i+1), w}-1}{2}=(-1) \frac{B(i)+1-w+a_{B(i), w}+1-1}{2}=-\operatorname{sign}(B(i), w)
$$

The case where $B(i+1)<w$ is completely analogous.

We conclude that within a block, rows $i$ and $i+1$ have opposite sign. The proof that within a block columns $j$ and $j+1$ have opposite sign is identical. So, within each block, the signs of the entries are staggered in a checkerboard pattern.

Since $M$ is a block matrix where the signs of each block are staggered in a checkerboard pattern, we can always choose rows and columns to multiply by -1 so that the signs of the matrix entries are staggered in a checkerboard pattern and the upper left entry is positive. Let $t$ be the total number of rows and columns we need to multiply by -1 . We claim that if node 1 is black, $(-1)^{t}=\operatorname{sign}_{\mathrm{c}}(\mathbf{N})(-1)^{\sum_{i=1}^{2 k}\left\lfloor\frac{n_{i}}{2}\right\rfloor}$ and if node 1 is white, $(-1)^{t}=(-1)^{n} \operatorname{sign}_{\mathrm{c}}(\mathbf{N})(-1)^{\sum_{i=1}^{2 k}\left\lfloor\frac{n_{i}}{2}\right\rfloor}$.

We will prove the claim by induction on $n$, where $2 n$ is the total number of nodes. The base case is when there are 4 nodes. In this case, $(-1)^{n}=1$. We check all possible node colorings in the table below. 


\begin{tabular}{|c|c|c|c|c|}
\hline black nodes & $M$ & $t$ & $\operatorname{sign}_{\mathrm{c}}(\mathbf{N})$ & $(-1)^{\sum\left\lfloor\frac{n_{i}}{2}\right\rfloor}$ \\
\hline 1,2 & $\left(\begin{array}{cc}-Y_{1,3} & Y_{1,4} \\
Y_{2,3} & -Y_{2,4}\end{array}\right)$ & 2 & -1 & -1 \\
\hline 1,3 & $\left(\begin{array}{cc}Y_{1,2} & -Y_{1,4} \\
-Y_{3,2} & Y_{3,4}\end{array}\right)$ & 0 & 1 & 1 \\
\hline 1,4 & $\left(\begin{array}{cc}Y_{1,2} & -Y_{1,3} \\
Y_{4,2} & -Y_{4,3}\end{array}\right)$ & 1 & 1 & -1 \\
\hline 3,4 & $\left(\begin{array}{cc}Y_{3,1} & -Y_{3,2} \\
-Y_{4,1} & Y_{4,2}\end{array}\right)$ & 0 & -1 & -1 \\
\hline 2,4 & $\left(\begin{array}{cc}-Y_{2,1} & Y_{2,3} \\
Y_{4,1} & -Y_{4,3}\end{array}\right)$ & 2 & 1 & 1 \\
\hline 2,3 & $\left(\begin{array}{cc}-Y_{2,1} & -Y_{2,4} \\
Y_{3,1} & Y_{3,4}\end{array}\right)$ & 1 & 1 & -1 \\
\hline
\end{tabular}

Now assume the claim holds when there are $2 n-2$ nodes and suppose that $|\mathbf{N}|=2 n$. Choose the largest nodes $x, x+1$ such that $x, x+1$ are different colors.

Let $\mathbf{N}^{\prime}=\{1, \ldots, 2 n-2\}$. Define $\psi: \mathbf{N}-\{x, x+1\} \rightarrow \mathbf{N}^{\prime}$ by

$$
\psi(\ell)=\left\{\begin{array}{ll}
\ell & \text { if } \ell<x \\
\ell-2 & \text { if } \ell>x+1
\end{array} .\right.
$$

That is, $\psi$ defines a relabeling of the nodes of $\mathbf{N}-\{x, x+1\}$ so that node 1 is labeled $1, \ldots$, node $x-1$ is labeled $x-1$, node $x+2$ is labeled $x, \ldots$, node $2 n$ is labeled $2 n-2$.

Recall that $\left(n_{1}, n_{1}+1\right), \ldots,\left(n_{2 k}, n_{2 k}+1\right)$ is a complete list of couples of consecutive nodes of the same color in $\mathbf{N}$. Let $\left(n_{1}^{\prime}, n_{1}^{\prime}+1\right), \ldots,\left(n_{2 j}^{\prime}, n_{2 j}^{\prime}+1\right)$ be a complete list of couples of consecutive nodes of the same color in $\mathbf{N}^{\prime}$.

Let $M^{\prime}$ denote the matrix corresponding to $\mathbf{N}^{\prime}$. Let $t^{\prime}$ denote the total number of rows and columns we need to multiply by -1 to get a matrix $M_{(1)}^{\prime}$ with entries whose signs are staggered in a checkerboard pattern so that the upper left entry is positive. By the induction hypothesis,

$$
(-1)^{t^{\prime}}=\operatorname{sign}_{\mathrm{c}}\left(\mathbf{N}^{\prime}\right)(-1)^{\sum_{i=1}^{2 j}\left\lfloor\frac{n_{i}^{\prime}}{2}\right\rfloor}
$$

if node 1 is black and

$$
(-1)^{t^{\prime}}=(-1)^{n-1} \operatorname{sign}_{\mathrm{c}}\left(\mathbf{N}^{\prime}\right)(-1)^{\sum_{i=1}^{2 j}\left\lfloor\frac{n_{i}^{\prime}}{2}\right\rfloor}
$$

if node 1 is white.

There are several cases to consider based on whether or not $\mathbf{N}$ and $\mathbf{N}^{\prime}$ have the same number of couples of consecutive nodes of the same color. In each case, we will assume that node $2 n$ is white. When $2 n$ is black, the argument is completely analogous.

Each case will involve two steps:

(i) comparing $\operatorname{sign}_{\mathrm{c}}\left(\mathbf{N}^{\prime}\right)$ to $\operatorname{sign}_{\mathrm{c}}(\mathbf{N})$ and $(-1)^{\sum\left\lfloor\frac{n_{i}^{\prime}}{2}\right\rfloor}$ to $(-1)^{\sum\left\lfloor\frac{n_{i}}{2}\right\rfloor}$, and

(ii) comparing $t$ to $t^{\prime}$.

Case 1. In the first case, $\mathbf{N}^{\prime}$ has the same number of couples of consecutive nodes of the same color as $\mathbf{N}$. There are two ways this can occur: $x+1<2 n$, or $x+1=2 n$.

Case 1(a). $x+1<2 n$

We first assume that node 1 is black.

(i) Comparing $\operatorname{sign}_{\mathbf{c}}(\mathbf{N})$ to $\operatorname{sign}_{\mathbf{c}}\left(\mathbf{N}^{\prime}\right)$ and $(-1)^{\sum\left\lfloor\frac{n_{i}^{\prime}}{2}\right\rfloor}$ to $(-1)^{\sum\left\lfloor\frac{n_{i}}{2}\right\rfloor}$.

Since $\mathbf{N}^{\prime}$ has the same number of couples of consecutive nodes as $\mathbf{N}, \operatorname{sign}_{\mathrm{c}}(\mathbf{N})=\operatorname{sign}_{\mathrm{c}}\left(\mathbf{N}^{\prime}\right)$. Since we assumed that $x$ and $x+1$ are the largest nodes such that $x$ and $x+1$ are different colors and $\mathbf{N}^{\prime}$ 

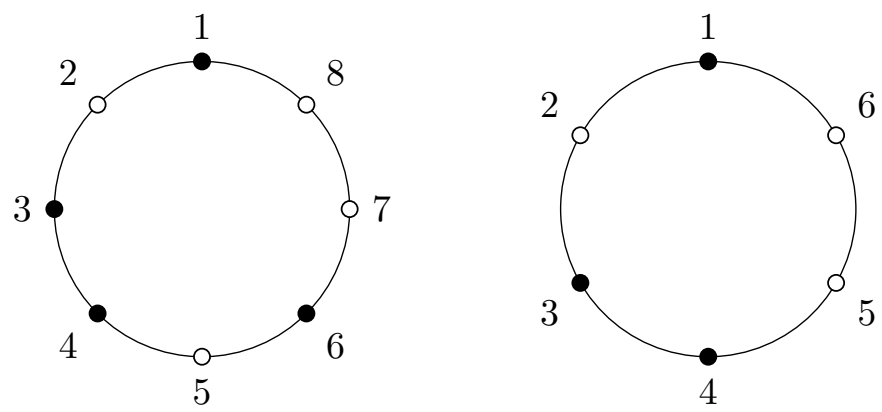

Figure 13. Shown left is an example of a possible node coloring $\mathbf{N}$ that could occur in Case 1(a). Nodes 6 and 7 are deleted from $\mathbf{N}$ and relabeled to obtain $\mathbf{N}^{\prime}$, which is shown right.

has the same number of couples of consecutive nodes as $\mathbf{N}$, node $x-1$ and all nodes in the interval $[x+1, \ldots, 2 n]$ are white. Since each node in the interval $[x+1, \ldots, 2 n]$ of $\mathbf{N}$ is white, each node in the interval $[x-1, \ldots, 2 n-2]$ of $\mathbf{N}$ is white, and node 1 is black in both $\mathbf{N}$ and $\mathbf{N}^{\prime}$ we have

$$
(-1)^{\sum_{i=1}^{2 k}\left\lfloor\frac{n_{i}^{\prime}}{2}\right\rfloor}=(-1)^{2 n-x-1}(-1)^{\sum_{i=1}^{2 k}\left\lfloor\frac{n_{i}}{2}\right\rfloor} \text {. }
$$

We conclude that

$$
\operatorname{sign}_{\mathrm{c}}\left(\mathbf{N}^{\prime}\right)(-1)^{\sum_{i=1}^{2 j}\left\lfloor\frac{n_{i}^{\prime}}{2}\right\rfloor}=(-1)^{2 n-x-1} \operatorname{sign}_{\mathrm{c}}(\mathbf{N})(-1)^{\sum_{i=1}^{2 j}\left\lfloor\frac{n_{i}}{2}\right\rfloor} .
$$

(ii) Comparing $t$ to $t^{\prime}$.

Comparing the parity of $t$ and $t^{\prime}$ is a multi-step process. Recall that we obtained $M_{(1)}^{\prime}$ from $M^{\prime}$ by multiplying $t^{\prime}$ rows and columns of $M^{\prime}$. We start by returning the nodes of $M_{(1)}^{\prime}$ to their original labels to obtain $M_{(2)}^{\prime}$. Then, we add the row and column corresponding to nodes $x$ and $x+1$ to $M_{(2)}^{\prime}$ to get $M_{(3)}^{\prime}$. Finally, we let $\widetilde{M}$ be the matrix obtained from $M$ by doing all the row and column multiplications we did to $M^{\prime}$ to get $M_{(1)}^{\prime}$, and consider the relationship between $\widetilde{M}$ and $M_{(3)}^{\prime}$.

Previously we defined the map $B:\{1,2, \ldots, n\} \rightarrow\left\{b_{1}, \ldots, b_{n}\right\}$ by letting $B(i)$ be the node corresponding to row $i$ of $M$ and we defined $W:\{1,2, \ldots, n\} \rightarrow\left\{w_{1}, \ldots, w_{n}\right\}$ by letting $W(j)$ be the node corresponding to column $j$ of $M$. It will be convenient to let $R:=B^{-1}$ and $C:=W^{-1}$, so for example $R(6)$ is the row corresponding to the black node 6 , and $C(7)$ is the column corresponding to the white node 7 .

We define $B^{\prime}, W^{\prime}, R^{\prime}$ and $C^{\prime}$ analogously for $M^{\prime}$.

Because this portion of the proof is long, we will illustrate the main ideas with an example. Let $G$ be a graph with 8 nodes where nodes $1,3,4$ and 6 are colored black (see Figure 13). In this example, $x=6$. So $\mathbf{N}^{\prime}=\{1,2,3,4,5,6\}$ where nodes 1,3 , and 4 are black. This means that

$$
M^{\prime}=\left(\begin{array}{ccc}
Y_{1,2} & Y_{1,5} & -Y_{1,6} \\
-Y_{3,2} & -Y_{3,5} & Y_{3,6} \\
Y_{4,2} & Y_{4,5} & -Y_{4,6}
\end{array}\right) .
$$

To obtain

$$
M_{(1)}^{\prime}=\left(\begin{array}{ccc}
Y_{1,2} & -Y_{1,5} & Y_{1,6} \\
-Y_{3,2} & Y_{3,5} & -Y_{3,6} \\
Y_{4,2} & -Y_{4,5} & Y_{4,6}
\end{array}\right)
$$

we multiply the second and third columns of $M$ by -1 , so $t^{\prime}=2$. 
In general, to get from $M^{\prime}$ to $M_{(1)}^{\prime}$, either we multiply all of the columns in a block or none of the columns in a block, because within each block, the signs of the entries are staggered in a checkerboard pattern. The same is true for the rows.

Return the nodes of $M_{(1)}^{\prime}$ to their original labels. Next, we return the nodes to their original labels (equivalently, we apply the map $\psi^{-1}$ ) to get $M_{(2)}^{\prime}$. Note that the only entries that are affected are the entries in the columns corresponding to nodes $\psi(x+2), \ldots, \psi(2 n)$.

In the example, we return node 6 to its original label of 8 , resulting in the matrix

$$
M_{(2)}^{\prime}=\left(\begin{array}{ccc}
Y_{1,2} & -Y_{1,5} & Y_{1,8} \\
-Y_{3,2} & Y_{3,5} & -Y_{3,8} \\
Y_{4,2} & -Y_{4,5} & Y_{4,8}
\end{array}\right)
$$

Add the row and column corresponding to nodes $x$ and $x+1$ to $M_{(2)}^{\prime}$. Now, add to $M_{(2)}^{\prime}$ the column corresponding to node $x+1$ (i.e. the column with entries $(-1)^{i>x+1} \operatorname{sign}(i, x+1) Y_{i, x+1}$ ) in between the columns corresponding to nodes $x-1$ and $x+2$. Also add the row corresponding to node $x$ as the last row. Change the sign of the entries in the new column in the rows of $M^{\prime}$ that we multiplied by -1 . Similarly, change the sign of the entries of the new row in the columns that we multiplied by -1 . Call the resulting matrix $M_{(3)}^{\prime}$.

In the example, we get

$$
M_{(3)}^{\prime}=\left(\begin{array}{cccc}
Y_{1,2} & -Y_{1,5} & -Y_{1,7} & Y_{1,8} \\
-Y_{3,2} & Y_{3,5} & Y_{3,7} & -Y_{3,8} \\
Y_{4,2} & -Y_{4,5} & -Y_{4,7} & Y_{4,8} \\
-Y_{6,2} & Y_{6,5} & Y_{6,7} & -Y_{6,8}
\end{array}\right)
$$

where note that we changed the sign of entries $Y_{6, \psi^{-1}(5)}=Y_{6,5}$ and $Y_{6, \psi^{-1}(6)}=Y_{6,8}$ because we multiplied the columns of $M^{\prime}$ corresponding to nodes 5 and 6 by -1 .

Since we changed the signs of entries in the row $R(x)$ and the column $C(x+1)$ as described above, $M_{(3)}^{\prime}$ is a block matrix with checkerboard blocks with the following additional properties:

(1) All columns strictly to the left of column $C(x+1)$ and all rows strictly above row $R(x)$ are in the same block.

(2) The $j$ th entry of $C(x-1)$ and $C(x+2)$ have opposite sign because they were adjacent in $M^{\prime}$, which is checkerboard.

(3) All columns strictly to the right of $C(x+1)$ are in the same block(s).

(4) $C(x+1)$ is either in same block as $C(x+2)$ or in the same block as $C(x-1)$.

(5) $R(x)$ is either in the same block as all other rows, or in its own block.

Compare $\widetilde{M}$ to $M_{(3)}^{\prime}$ and conclusion. Observe that if $i<x$ and $j>x+1$, then

$$
\operatorname{sign}(\psi(i), \psi(j))=(-1)^{\left(\psi(j)-\psi(i)+a_{\psi(i), \psi(j)}-1\right) / 2}=(-1)^{\left(j-i-2+a_{i, j}-1\right) / 2}=-\operatorname{sign}(i, j),
$$

so the entries in the columns $C(x+2), \ldots, C(2 n)$ are opposite in sign in $M$ compared to the entries in columns $C^{\prime}(\psi(x+2)), \ldots, C^{\prime}(\psi(2 n))$ in $M^{\prime}$.

Returning to our example, we see that

$$
M=\left(\begin{array}{cccc}
Y_{1,2} & Y_{1,5} & -Y_{1,7} & Y_{1,8} \\
-Y_{3,2} & -Y_{3,5} & Y_{3,7} & -Y_{3,8} \\
Y_{4,2} & Y_{4,5} & -Y_{4,7} & Y_{4,8} \\
-Y_{6,2} & -Y_{6,5} & Y_{6,7} & -Y_{6,8}
\end{array}\right) \text { and } M^{\prime}=\left(\begin{array}{ccc}
Y_{1,2} & Y_{1,5} & -Y_{1,6} \\
-Y_{3,2} & -Y_{3,5} & Y_{3,6} \\
Y_{4,2} & Y_{4,5} & -Y_{4,6}
\end{array}\right) \text {, }
$$

so indeed each entry in column $C(8)=4$ has sign opposite of the corresponding entry of column $C(6)=3$. 
Now let $\widetilde{M}$ be the matrix $M$ obtained by doing all of the $t^{\prime}$ row and column multiplications we did to $M^{\prime}$ to obtain $M_{(1)}^{\prime}$. In our example,

$$
\widetilde{M}=\left(\begin{array}{cccc}
Y_{1,2} & -Y_{1,5} & -Y_{1,7} & -Y_{1,8} \\
-Y_{3,2} & Y_{3,5} & Y_{3,7} & Y_{3,8} \\
Y_{4,2} & -Y_{4,5} & -Y_{4,7} & -Y_{4,8} \\
-Y_{6,2} & Y_{6,5} & Y_{6,7} & Y_{6,8}
\end{array}\right) .
$$

Since we changed the signs of entries in the row $R(x)$ and the column $C(x+1)$ as described in the previous step, by equation (3.1.2) $\widetilde{M}$ is identical to $M_{(3)}^{\prime}$ except for the columns $C(x+2), \ldots, C(2 n)$. Combining this fact with observations (1), (2), and (3) about $M_{(3)}^{\prime}$ above, we conclude that that $\widetilde{M}$ is checkerboard except possibly for the row $R(x)$ and column $C(x+1)$. Both the entries in $R(x)$ and the entries in $C(x+1)$ alternate in signs, so it remains to determine whether or not we need to multiply $R(x)$ and/or $C(x+1)$ by -1 .

Since $(-1)^{x>x+1}=1$ and $\operatorname{sign}(x+1, x)=1$, the entry $(R(x), C(x+1))$ of $\widetilde{M}$ is positive. Also, since in a matrix with checkerboard entries, the entry $(R(x), C(2 n))$ has positive sign, and all nodes $x+1, \ldots, 2 n$ are white, the entry $(R(x), C(x+1))$ of the final checkerboard matrix we get after multiplying $R(x)$ and/or $C(x+1)$ by -1 has positive sign if and only if $x$ is odd. This means that $x$ is odd if and only if we must multiply both $R(x)$ and $C(x+1)$ by -1 or neither by -1 to achieve a checkerboard matrix.

We conclude that $x$ is odd if and only if the parity of $t$ is the same as the parity of $t^{\prime}$. Since $x$ is odd if and only if $(-1)^{2 n-x-1}=1$, by equation (3.1.1), $t$ has the same parity as

$$
\operatorname{sign}_{\mathrm{c}}(\mathbf{N})(-1)^{\sum_{i=1}^{2 j}\left\lfloor\frac{n_{i}}{2}\right\rfloor}
$$

as desired.

When node 1 is white, the argument is very similar, but we have

$$
(-1)^{\sum_{i=1}^{2 j}\left\lfloor\frac{n_{i}^{\prime}}{2}\right\rfloor}=(-1)^{2 n-x}(-1)^{\sum_{i=1}^{2 j}\left\lfloor\frac{n_{i}}{2}\right\rfloor}
$$

since $(8,1)$ is a couple of consecutive nodes of the same color. It follows that

$$
(-1)^{n-1} \operatorname{sign}_{\mathrm{c}}\left(\mathbf{N}^{\prime}\right)(-1)^{\sum_{i=1}^{2 j}\left\lfloor\frac{n_{i}^{\prime}}{2}\right\rfloor}=(-1)^{n}(-1)^{2 n-x-1} \operatorname{sign}_{\mathrm{c}}(\mathbf{N})(-1)^{\sum_{i=1}^{2 j}\left\lfloor\frac{n_{i}}{2}\right\rfloor} .
$$

The rest of the argument is identical.

Case 1(b). $x+1=2 n$.

If $\mathbf{N}^{\prime}$ has the same number of couples of consecutive nodes as $\mathbf{N}$ and $x+1=2 n$, there are two possibilities: either nodes $2 n-2$ and 1 are black, or nodes $2 n-2$ and 1 are white.

We first assume that node 1 is black.

(i) Comparing $\operatorname{sign}_{\mathbf{c}}(\mathbf{N})$ to $\operatorname{sign}_{\mathbf{c}}\left(\mathbf{N}^{\prime}\right)$ and $(-1)^{\sum\left\lfloor\frac{n_{i}^{\prime}}{2}\right\rfloor}$ to $(-1)^{\sum\left\lfloor\frac{n_{i}}{2}\right\rfloor}$.

Since $\mathbf{N}^{\prime}$ has the same number of couples of consecutive nodes as $\mathbf{N}$ and $x+1=2 n, \operatorname{sign}_{\mathrm{c}}(\mathbf{N})=$ $\operatorname{sign}_{\mathrm{c}}\left(\mathbf{N}^{\prime}\right)$ and

$$
(-1)^{\sum_{i=1}^{2 k}\left\lfloor\frac{n_{i}^{\prime}}{2}\right\rfloor}=(-1)^{\sum_{i=1}^{2 k}\left\lfloor\frac{n_{i}}{2}\right\rfloor},
$$

so

(ii) Comparing $t$ to $t^{\prime}$.

$$
(-1)^{\sum_{i=1}^{2 k}\left\lfloor\frac{n_{i}}{2}\right\rfloor} \operatorname{sign}_{\mathrm{c}}(\mathbf{N})=(-1)^{\sum_{i=1}^{2 k}\left\lfloor\frac{n_{i}^{\prime}}{2}\right\rfloor} \operatorname{sign}_{\mathrm{c}}\left(\mathbf{N}^{\prime}\right) .
$$


In this case $\psi$ is the identity map, so $M_{(1)}^{\prime}=M_{(2)}^{\prime}$. We obtain $M_{(3)}^{\prime}$ as described in Case 1(a). By the same reasoning as in Case 1(a), all columns to the left of column $C(2 n)$ and all rows above row $R(2 n-1)$ are in the same block. $C(2 n)$ is either in the same block as the other columns or in its own block, and similarly for row $R(2 n-1)$.

Let $\widetilde{M}$ be the matrix $M$ obtained by doing all of the $t^{\prime}$ multiplications we did to $M^{\prime}$ to obtain $M_{(3)}^{\prime}$. Since $\psi$ is the identity map, $\widetilde{M}=M_{(3)}^{\prime}$. It remains to determine whether or not we need to multiply $R(2 n-1)$ and/or $C(2 n)$ by -1 .

Since $(-1)^{2 n-1>2 n}=1$ and $\operatorname{sign}(2 n-1,2 n)=1$, we need to multiply both $C(2 n)$ and $R(2 n-1)$ or neither in order for $\widetilde{M}$ to be checkerboard. So $t$ has the same parity as $t^{\prime}$ and therefore the same parity as

$$
(-1)^{\sum_{i=1}^{2 k}\left\lfloor\frac{n_{i}}{2}\right\rfloor} \operatorname{sign}_{\mathrm{c}}(\mathbf{N})
$$

This proves the claim when node 1 is black. If node 1 is white, the only difference is that

$$
(-1)^{\sum_{i=1}^{2 k}\left\lfloor\frac{n_{i}^{\prime}}{2}\right\rfloor}=-(-1)^{\sum_{i=1}^{2 k}\left\lfloor\frac{n_{i}}{2}\right\rfloor}
$$

because node $2 n$ was the first in a couple of consecutive white nodes in $\mathbf{N}$, and in $\mathbf{N}^{\prime}$, node $2 n-2$ is the first in a couple of consecutive white nodes. It follows that

$$
(-1)^{n-1}(-1)^{\sum_{i=1}^{2 k}\left\lfloor\frac{n_{i}^{\prime}}{2}\right\rfloor} \operatorname{sign}_{\mathrm{c}}\left(\mathbf{N}^{\prime}\right)=(-1)^{n}(-1)^{\sum_{i=1}^{2 k}\left\lfloor\frac{n_{i}}{2}\right\rfloor} \operatorname{sign}_{\mathrm{c}}(\mathbf{N}) .
$$

The rest of the proof is the same.
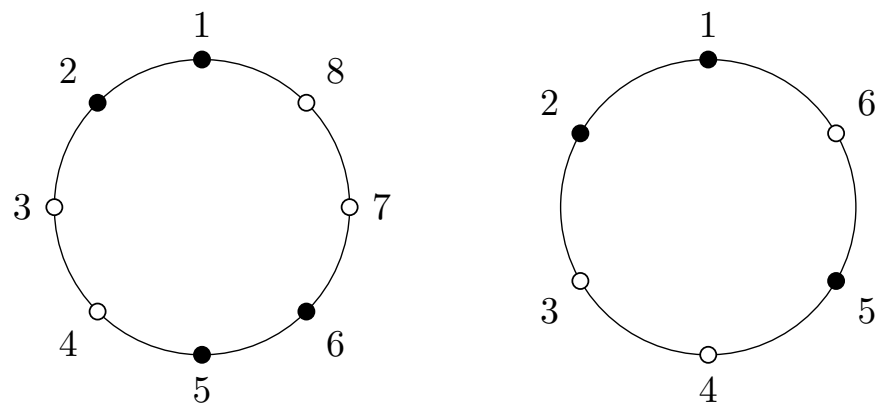

Figure 14. Shown left is an example of a possible node coloring $\mathbf{N}$ that could occur in Case 2(a). Nodes 6 and 7 are deleted from $\mathbf{N}$ and relabeled to obtain $\mathbf{N}^{\prime}$, which is the image on the right.

Case 2. In the second case, $\mathbf{N}^{\prime}$ has two fewer couples of consecutive nodes of the same color compared to $\mathbf{N}$. Again, there are two ways this can occur: $x+1<2 n$, or $x+1=2 n$.

Case 2(a). $x+1<2 n$

We first assume that node 1 is black. As in Case 1(a), we illustrate the main ideas with an example. Let $G$ be a graph with 8 nodes where nodes 1,2, 5 and 6 are colored black (see Figure 14). In this example, $x=6$, so $\mathbf{N}^{\prime}=\{1,2,3,4,5,6\}$ where nodes 1,2 , and 5 are black.

(i) Comparing $\operatorname{sign}_{\mathbf{c}}(\mathbf{N})$ to $\operatorname{sign}_{\mathbf{c}}\left(\mathbf{N}^{\prime}\right)$ and $(-1)^{\sum\left\lfloor\frac{n_{i}^{\prime}}{2}\right\rfloor}$ to $(-1)^{\sum\left\lfloor\frac{n_{i}}{2}\right\rfloor}$.

Since we assumed $\mathbf{N}^{\prime}$ has two fewer couples of consecutive nodes of the same color compared to $\mathbf{N}$, nodes $x-1$ and $x$ are both black.

Recall that $\left(s_{i}, s_{i}+1\right)$ denotes a couple of consecutive black nodes of the same color and $\left(u_{i}, u_{i}+1\right)$ denotes a couple of consecutive white nodes of the same color. By our assumptions, we have

$$
\cdots<s_{k}<u_{k-(2 n-x-2)}<\cdots<u_{k-1}<u_{k} .
$$


When we remove nodes $x$ and $x+1$, there is a one-to-one correspondence between the inversions with respect to the node coloring of $\mathbf{N}$ and the inversions with respect to the node coloring of $\mathbf{N}^{\prime}$ except for the inversions in $\mathbf{N}$ of the form $\left(s_{k}, u_{i}\right)$ for $k-(2 n-x-2) \leq i \leq k$. Thus we have that

$$
\operatorname{sign}_{\mathrm{c}}(\mathbf{N})=(-1)^{2 n-x-1} \operatorname{sign}_{\mathrm{c}}\left(\mathbf{N}^{\prime}\right) \text {. }
$$

In our example, in $\mathbf{N}$ we have $s_{1}<u_{1}<s_{2}<u_{2}$ and in $\mathbf{N}^{\prime}$ we have $s_{1}^{\prime}<u_{1}^{\prime}$. There is one fewer

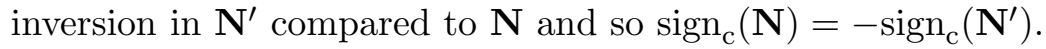

Next we compare $(-1)^{\sum_{i=1}^{2 k-2}\left\lfloor\frac{n_{i}^{\prime}}{2}\right\rfloor}$ to $(-1)^{\sum_{i=1}^{2 k}\left\lfloor\frac{n_{i}}{2}\right\rfloor}$. In our example, nodes 5 and 7 (nodes $x-1$ and $x+1$, respectively) are in $\left\{n_{1}, \ldots, n_{2 k}\right\}$. In $\mathbf{N}^{\prime}$, the black node $\psi(5)=5$ is adjacent to the white node $\psi(8)=6$ and the couple of consecutive white nodes $(7,8)$ is not replaced by a new couple of consecutive nodes, so

$$
(-1)^{\sum_{i=1}^{2 k}\left\lfloor\frac{n_{i}}{2}\right\rfloor}=(-1)^{\left\lfloor\frac{1}{2}\right\rfloor}(-1)^{\left\lfloor\frac{3}{2}\right\rfloor}(-1)^{\left\lfloor\frac{5}{2}\right\rfloor}(-1)^{\left\lfloor\frac{7}{2}\right\rfloor}=1
$$

while $(-1)^{\sum_{i=1}^{2 k-2}\left\lfloor\frac{n_{i}^{\prime}}{2}\right\rfloor}=(-1)^{\left\lfloor\frac{1}{2}\right\rfloor}(-1)^{\left\lfloor\frac{3}{2}\right\rfloor}=-1$.

In general, since the nodes $x+1, \ldots, 2 n$ are white and $x-1$ is black, the node $x-1$ and all nodes in the interval $[x+1, \ldots, 2 n-1]$ are equal to $n_{i}$ for some $i$. In $\mathbf{N}^{\prime}$, all nodes in the interval $[\psi(x+2), \ldots, \psi(2 n-1)]$ are equal to $n_{i}^{\prime}$ for some $i$.

From the observations that

- to obtain $\mathbf{N}^{\prime}$ we deleted nodes $x$ and $x+1$ from $\mathbf{N}$,

- $\psi(x-1)$ is adjacent to the white node $\psi(x+2)$ in $\mathbf{N}^{\prime}$, and

- $\psi(y)=y-2$ for $y>x+1$,

we get

$$
(-1)^{\sum_{i=1}^{2 k-2}\left\lfloor\frac{n_{i}^{\prime}}{2}\right\rfloor}=(-1)^{2 n-x-2}(-1)^{\left\lfloor\frac{x-1}{2}\right\rfloor}(-1)^{\left\lfloor\frac{x+1}{2}\right\rfloor}(-1)^{\sum_{i=1}^{2 k}\left\lfloor\frac{n_{i}}{2}\right\rfloor} .
$$

It follows that

so we conclude that

$$
(-1)^{\sum_{i=1}^{2 k-2}\left\lfloor\frac{n_{i}^{\prime}}{2}\right\rfloor}=(-1)^{x+1}(-1)^{\sum_{i=1}^{2 k}\left\lfloor\frac{n_{i}}{2}\right\rfloor}
$$

$$
\operatorname{sign}_{\mathrm{c}}\left(\mathbf{N}^{\prime}\right)(-1)^{\sum_{i=1}^{2 k-2}\left\lfloor\frac{n_{i}^{\prime}}{2}\right\rfloor}=\operatorname{sign}_{\mathrm{c}}(\mathbf{N})(-1)^{\sum_{i=1}^{2 k}\left\lfloor\frac{n_{i}}{2}\right\rfloor} .
$$

(ii) Comparing $t$ to $t^{\prime}$. This portion of the proof is similar in structure to part (ii) of Case 1(a). In our example,

$$
M^{\prime}=\left(\begin{array}{ccc}
-Y_{1,3} & Y_{1,4} & -Y_{1,6} \\
Y_{2,3} & -Y_{2,4} & Y_{2,6} \\
Y_{5,3} & -Y_{5,4} & Y_{5,6}
\end{array}\right)
$$

We multiply all three columns and the last row of $M^{\prime}$ by -1 to obtain $M_{(1)}^{\prime}$ and return node 6 to its original label of 8 to obtain $M_{(2)}^{\prime}$, so

$$
M_{(1)}^{\prime}=\left(\begin{array}{ccc}
Y_{1,3} & -Y_{1,4} & Y_{1,6} \\
-Y_{2,3} & Y_{2,4} & -Y_{2,6} \\
Y_{5,3} & -Y_{5,4} & Y_{5,6}
\end{array}\right) \text { and } M_{(2)}^{\prime}=\left(\begin{array}{ccc}
Y_{1,3} & -Y_{1,4} & Y_{1,8} \\
-Y_{2,3} & Y_{2,4} & -Y_{2,8} \\
Y_{5,3} & -Y_{5,4} & Y_{5,8}
\end{array}\right) .
$$

Add the column and row corresponding to nodes $x+1$ and $x$ to $M_{(2)}^{\prime}$. Now, add the column corresponding to node $x+1$ immediately to the left of the column corresponding to the node $x+2$ in $M_{(2)}^{\prime}$. Also add the row corresponding to node $x$ as the last row. Change the sign of the entries in the new column in rows $R(a)$ if $R^{\prime}(\psi(a))$ was a row we multiplied by -1 . Similarly, change the sign of the entries in the new row in columns $C(b)$ if $C^{\prime}(\psi(b))$ was a column we multiplied by -1 , and 
call the resulting matrix $M_{(3)}^{\prime}$, which is a block matrix with checkerboard blocks with properties (1) and (3)-(5) from Case 1(a). Property (2) has to be slightly modified:

(2) The $j$ th entry of the first column to the left of $C(x+1)$ and the $j$ th entry of $C(x+2)$ have opposite sign because they were adjacent in $M^{\prime}$, which is checkerboard.

In our example,

$$
M_{(3)}^{\prime}=\left(\begin{array}{cccc}
Y_{1,3} & -Y_{1,4} & Y_{1,7} & Y_{1,8} \\
-Y_{2,3} & Y_{2,4} & -Y_{2,7} & -Y_{2,8} \\
Y_{5,3} & -Y_{5,4} & Y_{5,7} & Y_{5,8} \\
Y_{6,3} & -Y_{6,4} & Y_{6,7} & Y_{6,8}
\end{array}\right)
$$

Compare $\widetilde{M}$ to the entries of $M_{(3)}^{\prime}$ and conclusion. Observe that if $i<x$ and $j>x+1$ then

$$
\operatorname{sign}(\psi(i), \psi(j))=(-1)^{\left(\psi(j)-\psi(i)+a_{\psi(i), \psi(j)}-1\right) / 2}=(-1)^{\left(j-2-i+a_{i, j}-2-1\right) / 2}=\operatorname{sign}(i, j),
$$

so unlike in Case 1(a), the entries in the columns $C(x+2), \ldots, C(2 n)$ are the same sign in $M$ as the entries in columns $C^{\prime}(\psi(x+2)), \ldots, C^{\prime}(\psi(2 n))$ in $M^{\prime}$.

Returning to our example, we see that the entries in column $C(8)=4$ have the same signs as the entries in column $C(6)=3$, as

$$
M=\left(\begin{array}{cccc}
-Y_{1,3} & Y_{1,4} & Y_{1,7} & -Y_{1,8} \\
Y_{2,3} & -Y_{2,4} & -Y_{2,7} & Y_{2,8} \\
Y_{5,3} & -Y_{5,4} & -Y_{5,7} & Y_{5,8} \\
-Y_{6,3} & Y_{6,4} & Y_{6,7} & -Y_{6,8}
\end{array}\right) \text { and } M^{\prime}=\left(\begin{array}{ccc}
-Y_{1,3} & Y_{1,4} & -Y_{1,6} \\
Y_{2,3} & -Y_{2,4} & Y_{2,6} \\
Y_{5,3} & -Y_{5,4} & Y_{5,6}
\end{array}\right)
$$

Let $\widetilde{M}$ be the matrix $M$ obtained by doing all of the $t^{\prime}$ multiplications we did to $M^{\prime}$ to obtain $M_{(3)}^{\prime}$. We see that $\widetilde{M}=M_{(3)}^{\prime}$, so $\widetilde{M}$ is checkerboard except for the columns $C(x+2), \ldots, C(2 n)$ and also possibly the row $R(x)$ and/or the column $C(x+1)$.

There are two cases to consider. In the first case, $C(x+1)$ is not in the same block as the first column to its left, so we need to multiply $C(x+1)$ by -1 . Then, since $C(x+1)$ is in the same block as $C(x+2), \ldots, C(2 n)$, we need to multiply the remaining $2 n-x-1$ columns by -1 as well. So we have done $t^{\prime}+2 n-x$ total multiplications. It remains to consider whether or not we need to multiply row $R(x)$ by -1 . Recall from Case 1(a) that after we are finished multiplying rows and columns and have obtained a checkerboard matrix, the entry $(R(x), C(x+1))$ must have positive sign if and only if $x$ is odd. Since $(-1)^{x>x+1}=1, \operatorname{sign}(x+1, x)=1$, and we multiplied $C(x+1)$ by -1 , we multiply $R(x)$ by -1 if and only if $x$ is odd. Therefore if $x$ is odd, we have done $t^{\prime}+2 n-x+1$ multiplications, and if $x$ is even, we have done $t^{\prime}+2 n-x$ multiplications. We have thus shown that $t$ has the same parity as $t^{\prime}$.

If $C(x+1)$ is in the same block as the first column to its left, we do not need to multiply $C(x+1)$ by -1 but we still need to multiply the remaining $2 n-x-1$ columns by -1 . So we have done $t^{\prime}+2 n-x-1$ total multiplications. Since we did not multiply $C(x+1)$ by -1 , we multiply $R(x)$ by -1 if and only if $x$ is even. Therefore, if $x$ is even, we have done $t^{\prime}+2 n-x$ total multiplications and if $x$ is odd we have done $t^{\prime}+2 n-x+1$ multiplications. Again, $t$ has the same parity as $t^{\prime}$.

In both cases, $t$ has the same parity as $\operatorname{sign}_{\mathrm{c}}(\mathbf{N})(-1)^{\sum_{i=1}^{2 k}\left\lfloor\frac{n_{i}}{2}\right\rfloor}$, which completes the proof when node 1 is black.

When node 1 is white, we have

$$
\cdots<s_{k}<u_{k-(2 n-x-1)}<\cdots<u_{k-1}<u_{k}
$$


but $\left(s_{k}, u_{k}\right)$ is not an inversion since node 1 is white. So we still have $\operatorname{sign}_{\mathrm{c}}(\mathbf{N})=(-1)^{2 n-x-1} \operatorname{sign}_{\mathrm{c}}\left(\mathbf{N}^{\prime}\right)$. Since $(2 n, 1)$ is a couple of consecutive white nodes,

$$
(-1)^{\sum_{i=1}^{2 k-2}\left\lfloor\frac{n_{i}^{\prime}}{2}\right\rfloor}=(-1)^{2 n-x-1}(-1)^{\left\lfloor\frac{x-1}{2}\right\rfloor}(-1)^{\left\lfloor\frac{x+1}{2}\right\rfloor}(-1)^{\sum_{i=1}^{2 k}\left\lfloor\frac{n_{i}}{2}\right\rfloor} .
$$

It follows that

$$
(-1)^{\sum_{i=1}^{2 k-2}\left\lfloor\frac{n_{i}^{\prime}}{2}\right\rfloor}=(-1)^{x}(-1)^{\sum_{i=1}^{2 k}\left\lfloor\frac{n_{i}}{2}\right\rfloor}
$$

so we conclude that

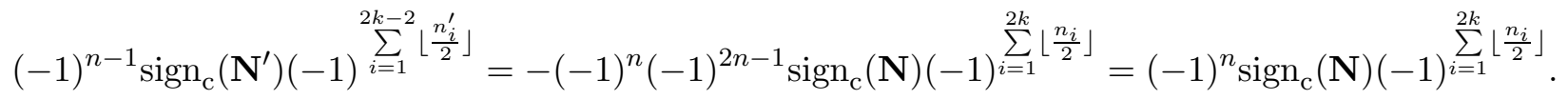

The rest of the argument is the same.

Case 2(b). $x+1=2 n$

If $\mathbf{N}^{\prime}$ has two fewer couples of consecutive nodes of the same color and compared to $\mathbf{N}$ and $x+1=2 n$, it must be the case that nodes $2 n-1$ and $2 n-2$ are both black and node 1 is white.

(i) Comparing $\operatorname{sign}_{\mathbf{c}}(\mathbf{N})$ to $\operatorname{sign}_{\mathbf{c}}\left(\mathbf{N}^{\prime}\right)$ and $(-1)^{\sum\left\lfloor\frac{n_{i}^{\prime}}{2}\right\rfloor}$ to $(-1)^{\sum\left\lfloor\frac{n_{i}}{2}\right\rfloor}$.

Removing nodes $2 n$ and $2 n-1$ does not remove any inversions with respect to the node coloring of $\mathbf{N}$ (recall that $\left(s_{k}, u_{k}\right)$ is not an inversion when node 1 is white). Thus $\operatorname{sign}_{\mathrm{c}}(\mathbf{N})=\operatorname{sign}_{\mathrm{c}}\left(\mathbf{N}^{\prime}\right)$.

Next observe that

$$
(-1)^{\sum_{i=1}^{2 k-2}\left\lfloor\frac{n_{i}^{\prime}}{2}\right\rfloor}=(-1)^{\left\lfloor\frac{2 n}{2}\right\rfloor}(-1)^{\left\lfloor\frac{2 n-2}{2}\right\rfloor}(-1)^{\sum_{i=1}^{2 k}\left\lfloor\frac{n_{i}}{2}\right\rfloor}=-(-1)^{\sum_{i=1}^{2 k}\left\lfloor\frac{n_{i}}{2}\right\rfloor} .
$$

Since node 1 is white, we have

$$
(-1)^{n-1} \operatorname{sign}_{\mathrm{c}}\left(\mathbf{N}^{\prime}\right)(-1)^{\sum_{i=1}^{2 j}\left\lfloor\frac{n_{i}^{\prime}}{2}\right\rfloor}=(-1)^{n} \operatorname{sign}_{\mathrm{c}}(\mathbf{N})(-1)^{\sum_{i=1}^{2 j}\left\lfloor\frac{n_{i}}{2}\right\rfloor} .
$$

\section{(ii) Comparing $t$ to $t^{\prime}$.}

This argument is identical to (ii) in Case 1(b), and we conclude that $t$ has the same parity as $t^{\prime}$, and therefore the same parity as $(-1)^{n} \operatorname{sign}_{\mathbf{c}}(\mathbf{N})(-1)^{\sum_{i=1}^{2 j}\left\lfloor\frac{n_{i}}{2}\right\rfloor}$.

Now that we have established Lemma 3.1.4, the proof of Theorem 1.3.2 is very similar to Kenyon and Wilson's proof of Theorem 1.2.1.

\section{Proof of Theorem 1.3.2.}

Without loss of generality ${ }^{9}$, we may assume that when we list the nodes in counterclockwise order starting with the red ones, they are in the order $1,2, \ldots, 2 n$. Combining Theorems 3.1.3 and 3.1.1 immediately gives a Pfaffian formula for the double-dimer model. For example, let $G$ be a graph with eight nodes where nodes $1,3,4$, and 6 are black. Assume the nodes are colored red, green and blue as shown to the right, so $\sigma=((1,8),(3,4),(5,6),(7,2))$. Then by Theorem 3.1.1,

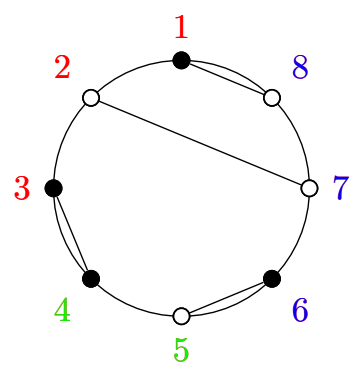




$$
\dddot{\operatorname{Pr}}(18|34| 56 \mid 72)=\left(\begin{array}{cccccccc}
0 & 0 & 0 & L_{1,4} & L_{1,5} & L_{1,6} & L_{1,7} & L_{1,8} \\
0 & 0 & 0 & L_{2,4} & L_{2,5} & L_{2,6} & L_{2,7} & L_{2,8} \\
0 & 0 & 0 & L_{3,4} & L_{3,5} & L_{3,6} & L_{3,7} & L_{3,8} \\
-L_{4,1} & -L_{4,2} & -L_{4,3} & 0 & 0 & L_{4,6} & L_{4,7} & L_{4,8} \\
-L_{5,1} & -L_{5,2} & -L_{5,3} & 0 & 0 & L_{5,6} & L_{5,7} & L_{5,8} \\
-L_{6,1} & -L_{6,2} & -L_{6,3} & -L_{6,4} & -L_{6,5} & 0 & 0 & 0 \\
-L_{7,1} & -L_{7,2} & -L_{7,3} & -L_{7,4} & -L_{7,5} & 0 & 0 & 0 \\
-L_{8,1} & -L_{8,2} & -L_{8,3} & -L_{8,4} & -L_{8,5} & 0 & 0 & 0
\end{array}\right)
$$

So making the substitution in Theorem 3.1.3 expresses $\widetilde{\operatorname{Pr}}(18|34| 56 \mid 72)$ as a Pfaffian, up to a global sign.

Presently, we explain how we can obtain a determinant formula from this Pfaffian formula. We make the substitution $L_{i, j} \rightarrow 0$ when $i$ and $j$ are both black or both white and we reorder the rows and columns so the black nodes are listed first. In the example, the above matrix becomes

$$
\left(\begin{array}{cccccccc}
0 & 0 & 0 & 0 & 0 & L_{1,5} & L_{1,7} & L_{1,8} \\
0 & 0 & 0 & 0 & 0 & L_{3,5} & L_{3,7} & L_{3,8} \\
0 & 0 & 0 & 0 & -L_{4,2} & 0 & L_{4,7} & L_{4,8} \\
0 & 0 & 0 & 0 & -L_{6,2} & -L_{6,5} & 0 & 0 \\
0 & 0 & L_{2,4} & L_{2,6} & 0 & 0 & 0 & 0 \\
-L_{5,1} & -L_{5,3} & 0 & L_{5,6} & 0 & 0 & 0 & 0 \\
-L_{7,1} & -L_{7,3} & -L_{7,4} & 0 & 0 & 0 & 0 & 0 \\
-L_{8,1} & -L_{8,3} & -L_{8,4} & 0 & 0 & 0 & 0 & 0
\end{array}\right) .
$$

Simultaneous swaps of two different rows and corresponding columns changes the sign of the Pfaffian. Assuming the graph has $2 k$ couples of consecutive nodes of the same color, we claim that the number of swaps needed so that the black nodes are listed first has the same parity as

$$
\frac{n(n-1)}{2}+\sum_{i=1}^{2 k}\left\lfloor\frac{n_{i}}{2}\right\rfloor,
$$

if node 1 is black. If node 1 is white, the number of swaps needed has the same parity as

$$
\frac{n(n+1)}{2}+\sum_{i=1}^{2 k}\left\lfloor\frac{n_{i}}{2}\right\rfloor .
$$

To prove this, we will first show that the number of node swaps needed to get from a node coloring with $2 k$ couples of consecutive nodes of the same color to a node coloring that alternates black and white has the same parity as $\sum_{i=1}^{2 k}\left\lfloor\frac{n_{i}}{2}\right\rfloor$.

We will prove this by induction on $k$. When $k=0,0$ swaps are needed, so the claim holds trivially. Assume the claim holds when $\mathbf{N}$ has $2(k-1)$ couples of consecutive nodes of the same color and suppose we have a set of nodes that has $2 k$ couples of consecutive nodes of the same color. Let $h$ be the smallest integer so that $n_{h-1}$ and $n_{h}$ are different colors. Then $n_{h-1}$ and $n_{h}$ are the same parity and there are an even number of nodes in the interval $\left[n_{h-1}+1, \ldots, n_{h}\right]$, which alternate in color. If we swap $n_{h}$ with $n_{h}-1, n_{h}-2$ with $n_{h}-3, \ldots, n_{h-1}+2$ with $n_{h-1}+1$, we will have done $\frac{n_{h}-n_{h-1}}{2}$ swaps and we will have a node coloring with $2(k-1)$ couples of consecutive

\footnotetext{
${ }^{9}$ We can renumber the nodes while preserving their cyclic order without changing the global sign of the Pfaffian in Theorem 3.1.1. This is because if we move the last row and column to be the first row and column, the sign of the Pfaffian changes. But since the entries above the diagonal must be non-negative, we negate the new first row and column and the Pfaffian changes sign again.
} 
nodes of the same color. If $n_{h}$ and $n_{h-1}$ are both even then $\frac{n_{h}-n_{h-1}}{2}$ clearly has the same parity as $\left\lfloor\frac{n_{h}}{2}\right\rfloor+\left\lfloor\frac{n_{h-1}}{2}\right\rfloor$. If $n_{h}$ and $n_{h-1}$ are both odd then by writing $\frac{n_{h}-n_{h-1}}{2}=\frac{n_{h}-1-\left(n_{h-1}-1\right)}{2}$ we see that $\frac{n_{h}-n_{h-1}}{2}$ and $\left\lfloor\frac{n_{h}}{2}\right\rfloor+\left\lfloor\frac{n_{h-1}}{2}\right\rfloor$ have the same parity. By the induction hypothesis, the number of swaps needed to get to a node coloring that alternates black and white has the same parity as

$$
\sum_{\substack{1 \leq i<2 k \\ i \neq h, h-1}}\left\lfloor\frac{n_{i}}{2}\right\rfloor
$$

The claim follows.

Assume node 1 is black. If there are no couples of consecutive nodes of the same color, the number of swaps needed to put the black nodes first is

$$
1+2+3+\cdots+(n-1)=\frac{n(n-1)}{2}
$$

because the third node requires 1 swap, the fifth node requires 2 swaps, the seventh node requires 3 swaps, $\ldots$, and the $(2 n-1)$ st node requires $n-1$ swaps. So if there are $2 k$ couples of consecutive nodes of the same color, since $\sum_{i=1}^{2 k}\left\lfloor\frac{n_{i}}{2}\right\rfloor$ node swaps are needed to get to a node coloring that alternates black and white, the number of swaps needed so that the black nodes are listed first has the same parity as

$$
\frac{n(n-1)}{2}+\sum_{i=1}^{2 k}\left\lfloor\frac{n_{i}}{2}\right\rfloor
$$

If node 1 is white, the number of swaps needed to put the black nodes first is

$$
1+2+3+\cdots+n=\frac{n(n+1)}{2}
$$

because the second node requires 1 swap, the fourth node requires 2 swaps, .., and the $(2 n)$ th node requires $n$ swaps. So the number of swaps needed so that the black nodes are listed first has the same parity as

$$
\frac{n(n+1)}{2}+\sum_{i=1}^{2 k}\left\lfloor\frac{n_{i}}{2}\right\rfloor
$$

Next, observe that after the rows and columns have been sorted, the matrix has the form

$$
\left(\begin{array}{cc}
0 & \pm L_{B, W} \\
\mp L_{W, B} & 0
\end{array}\right)
$$

where $B$ represents the black nodes, $W$ the white nodes, and the signs of the entries in $\pm L_{B, W}$ are + if the black node has a smaller label than the white node and - otherwise. The Pfaffian of this matrix is the determinant of the upper right submatrix times $(-1)^{\frac{n(n-1)}{2}}$. To summarize, after making the substitution $L_{i, j} \rightarrow 0$ when $i$ and $j$ are both black or both white and sorting the rows and columns so the black nodes are listed first,

$$
\operatorname{Pf}\left(\begin{array}{ccc}
0 & L_{R, G} & L_{R, B} \\
-L_{G, R} & 0 & L_{G, B} \\
-L_{B, R} & -L_{B, G} & 0
\end{array}\right)=(-1)^{\sum_{i=1}^{2 k}\left\lfloor\frac{n_{i}}{2}\right\rfloor} \operatorname{det}\left(L_{B, W}\right)
$$

when node 1 is black. When node 1 is white,

$$
\operatorname{Pf}\left(\begin{array}{ccc}
0 & L_{R, G} & L_{R, B} \\
-L_{G, R} & 0 & L_{G, B} \\
-L_{B, R} & -L_{B, G} & 0
\end{array}\right)=(-1)^{n}(-1)^{\sum_{i=1}^{2 k}\left\lfloor\frac{n_{i}}{2}\right\rfloor} \operatorname{det}\left(L_{B, W}\right) .
$$


In the example, after this substitution and reordering, the Pfaffian of matrix (3.1.3) is equal to

$$
(-1)^{1+3} \operatorname{det}\left(\begin{array}{cccc}
0 & L_{1,5} & L_{1,7} & L_{1,8} \\
0 & L_{3,5} & L_{3,7} & L_{3,8} \\
-L_{4,2} & 0 & L_{4,7} & L_{4,8} \\
-L_{6,2} & -L_{6,5} & 0 & 0
\end{array}\right)
$$

because $n_{1}=3$ and $n_{2}=7$.

Next we do the substitution $L_{i, j} \rightarrow \operatorname{sign}(i, j) Y_{i, j}$. The result is the matrix

$$
M=\left[1_{i, j} \text { RGB-colored differently }(-1)^{i>j} \operatorname{sign}(i, j) Y_{i, j}\right]_{j=w_{1}, w_{2}, \ldots, w_{n}}^{i=b_{1}, b_{2}, \ldots, b_{n}}
$$

where $b_{1}<b_{2}<\cdots<b_{n}$ are the black nodes listed in increasing order and $w_{1}<w_{2}<\cdots<w_{n}$ are the white nodes listed in increasing order. By Theorem 3.1.3,

$$
\widetilde{\operatorname{Pr}}(\sigma)=\operatorname{sign}_{O E}(\sigma) \operatorname{sign}_{\mathrm{c}}(\mathbf{N})(-1)^{\sum_{i=1}^{2 k}\left\lfloor\frac{n_{i}}{2}\right\rfloor} \operatorname{det}(M)
$$

if node 1 is black and

$$
\widetilde{\operatorname{Pr}}(\sigma)=\operatorname{sign}_{O E}(\sigma) \operatorname{sign}_{\mathrm{c}}(\mathbf{N})(-1)^{n}(-1)^{\sum_{i=1}^{2 k}\left\lfloor\frac{n_{i}}{2}\right\rfloor} \operatorname{det}(M)
$$

is node 1 is white. By Lemma 3.1.4, $M$ is a block matrix where within each block, the signs of the entries are staggered in a checkerboard pattern. Next, we multiply rows and columns of $M$ by -1 so that the signs of the matrix entries are staggered in a checkerboard pattern and the upper left entry is positive. Call the resulting matrix $\widetilde{M}$. By Lemma 3.1.4,

$$
\widetilde{\operatorname{Pr}}(\sigma)=\operatorname{sign}_{O E}(\sigma) \operatorname{det}(\widetilde{M})
$$

regardless of whether node 1 is black or white. Then, if we multiply every other row by -1 and every other column by -1 , the signs of all matrix entries are positive and the determinant is unchanged. We conclude that

$$
\widetilde{\operatorname{Pr}}(\sigma)=\operatorname{sign}_{O E}(\sigma) \operatorname{det}\left[1_{i, j} \text { RGB-colored differently } Y_{i, j}\right]_{j=w_{1}, w_{2}, \ldots, w_{n}}^{i=b_{1}, b_{2}, \ldots, b_{n}} .
$$

Returning to our example, we find that

$\widetilde{\operatorname{Pr}}(18|34| 56 \mid 72)=\operatorname{sign}_{O E}(18|34| 56 \mid 72) \operatorname{det}\left(\begin{array}{cccc}0 & Y_{1,5} & Y_{1,7} & Y_{1,8} \\ 0 & Y_{3,5} & Y_{3,7} & Y_{3,8} \\ Y_{4,2} & 0 & Y_{4,7} & Y_{4,8} \\ Y_{6,2} & Y_{6,5} & 0 & 0\end{array}\right)=-\operatorname{det}\left(\begin{array}{cccc}0 & Y_{1,5} & Y_{1,7} & Y_{1,8} \\ 0 & Y_{3,5} & Y_{3,7} & Y_{3,8} \\ Y_{4,2} & 0 & Y_{4,7} & Y_{4,8} \\ Y_{6,2} & Y_{6,5} & 0 & 0\end{array}\right)$.

3.2. Proof of Theorem 1.3.3. Now that we have established Theorem 1.3.2, Theorem 1.3.3 follows from the proof method described in Section 1.2.

Theorem 1.3.3. Let $G=\left(V_{1}, V_{2}, E\right)$ be a finite edge-weighted planar bipartite graph with a set of nodes $\mathbf{N}$. Divide the nodes into three circularly contiguous sets $R, G$, and $B$ such that $|R|,|G|$, and $|B|$ satisfy the triangle inequality and let $\sigma$ be the corresponding tripartite pairing. If $x, w \in V_{1}$ and $y, v \in V_{2}$ then

$$
\begin{aligned}
& \operatorname{sign}_{O E}(\sigma) \operatorname{sign}_{O E}\left(\sigma_{x y w v}^{\prime}\right) Z_{\sigma}^{D D}(G, \mathbf{N}) Z_{\sigma_{x y w v}}^{D D}(G, \mathbf{N}-\{x, y, w, v\}) \\
= & \operatorname{sign}_{O E}\left(\sigma_{x y}^{\prime}\right) \operatorname{sign}_{O E}\left(\sigma_{w v}^{\prime}\right) Z_{\sigma_{x y}}^{D D}(G, \mathbf{N}-\{x, y\}) Z_{\sigma_{w v}}^{D D}(G, \mathbf{N}-\{w, v\}) \\
& -\operatorname{sign}_{O E}\left(\sigma_{x v}^{\prime}\right) \operatorname{sign}_{O E}\left(\sigma_{w y}^{\prime}\right) Z_{\sigma_{x v}}^{D D}(G, \mathbf{N}-\{x, v\}) Z_{\sigma_{w y}}^{D D}(G, \mathbf{N}-\{w, y\})
\end{aligned}
$$

where for $i, j \in\{x, y, w, v\}, \sigma_{i j}$ is the unique planar pairing on $\mathbf{N}-\{i, j\}$ in which like colors are not paired together, and $\sigma_{i j}^{\prime}$ is the pairing after the the node set $\mathbf{N}-\{i, j\}$ has been relabeled so that the nodes are numbered consecutively. 
Proof. First we assume that all pairings in the theorem statement exist. Let

$$
M=\left[1_{i, j} \text { RGB-colored differently } Y_{i, j}\right]_{j=w_{1}, w_{2}, \ldots, w_{n}}^{i=b_{1}, b_{2}, \ldots, b_{n}}
$$

and let $r_{x}$ and $r_{w}$ denote the rows corresponding to nodes $x$ and $w$, respectively (so we are assuming that $x$ and $w$ are colored black).

We first move the columns corresponding to $y$ and $v$ (i.e. the columns with entries $Y_{i, y}$ and $Y_{i, v}$, respectively) to the columns $r_{x}$ and $r_{w}$. We observe that we can do this without exchanging the column with entries $Y_{i, y}$ with the column with entries $Y_{i, v}$. For example, if $r_{x}<c_{y}<r_{w}<c_{v}$, we swap column $c_{y}$ with column $c_{y}-1$, then column $c_{y}-1$ with column $c_{y}-2, \ldots$, and column $r_{x}+1$ with column $r_{x}$. Next, we swap column $c_{v}$ with column $c_{v}-1, \ldots$, and column $r_{w}+1$ with column $r_{w}$. If instead $c_{y}<c_{v}<r_{x}<r_{w}$, we swap column $c_{v}$ with column $c_{v}+1, \ldots$, and column $r_{w}-1$ with column $r_{w}$ before swapping $c_{y}$ with column $c_{y}+1, \ldots$, and column $r_{x}-1$ with column $r_{x}$.

Without loss of generality we assume that we move the column with entries $Y_{i, y}$ to column $r_{x}$ and the column with entries $Y_{i, v}$ to column $r_{w}$ to obtain the matrix $\widetilde{M}$. Let $s_{y}$ denote the number of column swaps we make in the process of moving the column with entries $Y_{i, y}$. Let $s_{v}$ denote the number of column swaps we make in the process of moving the column with entries $Y_{i, v}$. Note that $s_{y}$ and $s_{v}$ are well-defined up to parity. Note also that after making these swaps, the columns are still in ascending order, aside from the columns with entries $Y_{i, y}$ and $Y_{i, v}$.

By the Desnanot-Jacobi identity,

$$
\operatorname{det}(\widetilde{M}) \operatorname{det}\left(\widetilde{M}_{r_{x}, r_{w}}^{r_{x}, r_{w}}\right)=\operatorname{det}\left(\widetilde{M}_{r_{x}}^{r_{x}}\right) \operatorname{det}\left(\widetilde{M}_{r_{w}}^{r_{w}}\right)-\operatorname{det}\left(\widetilde{M}_{r_{w}}^{r_{x}}\right) \operatorname{det}\left(\widetilde{M}_{r_{x}}^{r_{w}}\right),
$$

where recall that $M_{s}^{t}$ is the matrix $M$ with row $s$ and column $t$ removed.

We apply Theorem 1.3.2 to each term in equation (3.2.1). First consider $\operatorname{det}\left(\widetilde{M}_{r_{x}}^{r_{x}}\right)$. In order to apply Theorem 1.3.2 we must reorder the columns. Since we have removed the column $r_{x}$ which had entries $Y_{i, y}, s_{v}$ column swaps will put the columns in the correct (ascending) order. This follows from the previous observation that we moved the columns corresponding to $y$ and $v$ without exchanging the column with entries $Y_{i, y}$ with the column with entries $Y_{i, v}$.

We must also relabel the nodes $\mathbf{N}-\{x, y\}$ so that they are numbered consecutively. Recall that $\sigma_{x y}$ denotes the unique planar pairing of $\mathbf{N}-\{x, y\}$ in which like colors are not paired together. When we relabel $\mathbf{N}-\{x, y\}$ we relabel $\sigma_{x y}$ as well. Call the resulting node set $\mathbf{N}^{\prime}$ and the resulting pairing $\sigma_{x y}^{\prime}$. Then by Theorem 1.3.2,

$$
\operatorname{det}\left(\widetilde{M}_{r_{x}}^{r_{x}}\right)=(-1)^{s_{v}} \operatorname{sign}_{O E}\left(\sigma_{x y}^{\prime}\right) \frac{Z_{\sigma_{x y}^{\prime}}^{D D}\left(G, \mathbf{N}^{\prime}\right)}{\left(Z^{D}(G)\right)^{2}},
$$

and thus

$$
\operatorname{det}\left(\widetilde{M}_{r_{x}}^{r_{x}}\right)=(-1)^{s_{v}} \operatorname{sign}_{O E}\left(\sigma_{x y}^{\prime}\right) \frac{Z_{\sigma_{x y}}^{D D}(G, \mathbf{N}-\{x, y\})}{\left(Z^{D}(G)\right)^{2}} .
$$

Similarly, we have

$$
\begin{aligned}
& \operatorname{det}\left(\widetilde{M}_{r_{w}}^{r_{w}}\right)=(-1)^{s_{y}} \operatorname{sign}_{O E}\left(\sigma_{w v}^{\prime}\right) \frac{Z_{\sigma_{w v}}^{D D}(G, \mathbf{N}-\{w, v\})}{\left(Z^{D}(G)\right)^{2}}, \\
& \operatorname{det}\left(\widetilde{M}_{r_{x}}^{r_{w}}\right)=(-1)^{s_{y}} \operatorname{sign}_{O E}\left(\sigma_{x v}^{\prime}\right) \frac{Z_{\sigma_{x v}}^{D D}(G, \mathbf{N}-\{x, v\})}{\left(Z^{D}(G)\right)^{2}}, \text { and } \\
& \operatorname{det}\left(\widetilde{M}_{r_{w}}^{r_{x}}\right)=(-1)^{s_{v}} \operatorname{sign}_{O E}\left(\sigma_{y w}^{\prime}\right) \frac{Z_{\sigma_{y w}}^{D D}(G, \mathbf{N}-\{y, w\})}{\left(Z^{D}(G)\right)^{2}} .
\end{aligned}
$$


It follows that the right hand side of equation (3.2.1) is

$$
\begin{aligned}
& (-1)^{s_{y}}(-1)^{s_{v}}\left(\operatorname{sign}_{O E}\left(\sigma_{x y}^{\prime}\right) \operatorname{sign}_{O E}\left(\sigma_{w v}^{\prime}\right) \frac{Z_{\sigma_{x y}}^{D D}(G, \mathbf{N}-\{x, y\})}{\left(Z^{D}(G)\right)^{2}} \frac{Z_{\sigma_{w v}}^{D D}(G, \mathbf{N}-\{w, v\})}{\left(Z^{D}(G)\right)^{2}}\right. \\
& \left.-\operatorname{sign}_{O E}\left(\sigma_{x v}^{\prime}\right) \operatorname{sign}_{O E}\left(\sigma_{y w}^{\prime}\right) \frac{Z_{\sigma_{x v}}^{D D}(G, \mathbf{N}-\{x, v\})}{\left(Z^{D}(G)\right)^{2}} \frac{Z_{\sigma_{y w}}^{D D}(G, \mathbf{N}-\{y, w\})}{\left(Z^{D}(G)\right)^{2}}\right) .
\end{aligned}
$$

Applying Theorem 1.3.2 to the left hand side of equation (3.2.1), we have

$$
\begin{aligned}
\operatorname{det}(\widetilde{M}) & =(-1)^{s_{y}}(-1)^{s_{v}} \operatorname{sign}_{O E}(\sigma) \frac{Z_{\sigma}^{D D}(G, \mathbf{N})}{\left(Z^{D}(G)\right)^{2}}, \text { and } \\
\operatorname{det}\left(\widetilde{M}_{r_{x}, r_{w}}^{r_{x}, r_{w}}\right) & =\operatorname{sign}_{O E}\left(\sigma_{x y w v}^{\prime}\right) \frac{Z_{\sigma_{x y w v}}^{D D}(G, \mathbf{N}-\{x, y, w, v\})}{\left(Z^{D}(G)\right)^{2}} .
\end{aligned}
$$

We conclude that

$$
\begin{aligned}
& \operatorname{sign}_{O E}(\sigma) \operatorname{sign}_{O E}\left(\sigma_{x y w v}^{\prime}\right) Z_{\sigma}^{D D}(G, \mathbf{N}) Z_{\sigma_{x y w v}}^{D D}(G, \mathbf{N}-\{x, y, w, v\}) \\
= & \operatorname{sign}_{O E}\left(\sigma_{x y}^{\prime}\right) \operatorname{sign}_{O E}\left(\sigma_{w v}^{\prime}\right) Z_{\sigma_{x y}}^{D D}(G, \mathbf{N}-\{x, y\}) Z_{\sigma_{w v}}^{D D}(G, \mathbf{N}-\{w, v\}) \\
& -\operatorname{sign}_{O E}\left(\sigma_{x v}^{\prime}\right) \operatorname{sign}_{O E}\left(\sigma_{w y}^{\prime}\right) Z_{\sigma_{x v}}^{D D}(G, \mathbf{N}-\{x, v\}) Z_{\sigma_{w y}}^{D D}(G, \mathbf{N}-\{w, y\}) .
\end{aligned}
$$

It is not necessarily the case that the pairings $\sigma_{x y}, \sigma_{w v}, \sigma_{x v}, \sigma_{w y}$, and $\sigma_{x y w v}$ all exist. First consider the case where one of the pairings $\sigma_{x y}, \sigma_{w v}, \sigma_{x v}, \sigma_{w y}$ does not exist. Without loss of generality, assume that $\sigma_{x y}$ does not exist. This means that the number of nodes of different colors in $\mathbf{N}-\{x, y\}$ do not satisfy the triangle inequality. Then $\operatorname{det}\left(\widetilde{M}_{r_{x}}^{r_{x}}\right)=0$ since every black-white pairing contains an $R G B$-monochromatic pair. There are two possibilities in this case: either the theorem statement holds trivially, or

$$
Z_{\sigma}^{D D}(G, \mathbf{N}) Z_{\sigma_{x y w v}}^{D D}(G, \mathbf{N}-\{x, y, w, v\})=Z_{\sigma_{x v}}^{D D}(G, \mathbf{N}-\{x, v\}) Z_{\sigma_{w y}}^{D D}(G, \mathbf{N}-\{w, y\}) .
$$

Since the numbers of nodes of different colors in $\mathbf{N}-\{x, y\}$ do not satisfy the triangle inequality, without loss of generality, we may assume there are more red nodes than the combined number of blue and green nodes in $\mathbf{N}-\{x, y\}$. Since we assumed that in $\mathbf{N},|R|,|G|$, and $|B|$ satisfy the triangle inequality, it follows that $|R|=|G|+|B|$ in N. Assuming without loss of generality that when we list the nodes in counterclockwise order starting with the red ones, they are in the order $1,2, \ldots, 2 n$, this means $\sigma$ is the pairing $((1,2 n),(2,2 n-1), \ldots,(n, n+1))$. It must be the case that $x$ and $y$ are both green or blue, so if either $w$ or $v$ is green, then $\sigma_{x y w v}$ does not exist and either $\sigma_{x v}$ or $\sigma_{w v}$ does not exist, so the equality holds trivially. If both $w$ and $v$ are red, then $\sigma_{x v}, \sigma_{w v}$ and $\sigma_{x y w v}$ all exist. In this case, the rest of the proof proceeds as above and we have

$$
\begin{aligned}
& \operatorname{sign}_{O E}(\sigma) \operatorname{sign}_{O E}\left(\sigma_{x y w v}^{\prime}\right) Z_{\sigma}^{D D}(G, \mathbf{N}) Z_{\sigma_{x y w v}}^{D D}(G, \mathbf{N}-\{x, y, w, v\}) \\
= & -\operatorname{sign}_{O E}\left(\sigma_{x v}^{\prime}\right) \operatorname{sign}_{O E}\left(\sigma_{w y}^{\prime}\right) Z_{\sigma_{x v}}^{D D}(G, \mathbf{N}-\{x, v\}) Z_{\sigma_{w y}}^{D D}(G, \mathbf{N}-\{w, y\}) .
\end{aligned}
$$

Recall that inversions in a planar pairing correspond to nestings (see Remark 2.1.18). Because $\sigma$ is the pairing $((1,2 n),(2,2 n-1), \ldots,(n, n+1)), \operatorname{sign}\left(\sigma_{x v}^{\prime}\right)=\operatorname{sign}\left(\sigma_{w y}^{\prime}\right)$ and $\operatorname{sign}\left(\sigma_{x y w v}^{\prime}\right)=\operatorname{sign}(\sigma)$. $(-1)^{n-1} \cdot(-1)^{n-2}$. Equation (3.2.4) follows.

If $\sigma_{x y w v}$ does not exist, then this means that the numbers of nodes of different colors in $\mathbf{N}-$ $\{x, y, w, v\}$ do not satisfy the triangle inequality. Without loss of generality, we may assume there are more red nodes than the combined number of blue and green nodes in $\mathbf{N}-\{x, y, w, v\}$. By the same reasoning as above, $\operatorname{det}\left(\widetilde{M}_{r_{x}}^{r_{x}, r_{w}}\right)=0$. If any one of $x, y, w$, or $v$ is red, then the equation holds trivially. If all of $x, y, w$, and $v$ are green or blue, then in the original node set $\mathbf{N},|R|+2=|G|+|B|$. 
So each of the pairings $\sigma_{x y}^{\prime}, \sigma_{w v}^{\prime}, \sigma_{x v}^{\prime}$, and $\sigma_{w y}^{\prime}$ is $((1,2 n-2),(2,2 n-1), \ldots)$. Then we have

$$
\begin{aligned}
& \operatorname{sign}_{O E}\left(\sigma_{x y}^{\prime}\right) \operatorname{sign}_{O E}\left(\sigma_{w v}^{\prime}\right) Z_{\sigma_{x y}}^{D D}(G, \mathbf{N}-\{x, y\}) Z_{\sigma_{w v}}^{D D}(G, \mathbf{N}-\{w, v\}) \\
= & \operatorname{sign}_{O E}\left(\sigma_{x v}^{\prime}\right) \operatorname{sign}_{O E}\left(\sigma_{w y}^{\prime}\right) Z_{\sigma_{x v}}^{D D}(G, \mathbf{N}-\{x, v\}) Z_{\sigma_{w y}}^{D D}(G, \mathbf{N}-\{w, y\}) .
\end{aligned}
$$

Since inversions in a planar pairing correspond to nestings, all pairings have the same sign. So in this case,

$$
Z_{\sigma_{x y}}^{D D}(G, \mathbf{N}-\{x, y\}) Z_{\sigma_{w v}}^{D D}(G, \mathbf{N}-\{w, v\})=Z_{\sigma_{x v}}^{D D}(G, \mathbf{N}-\{x, v\}) Z_{\sigma_{w y}}^{D D}(G, \mathbf{N}-\{w, y\}) .
$$

Remark 3.2.1. To simplify the expression in Theorem 1.3.3, it suffices to know the RGB-coloring of the nodes $x, y, w, v$.

Without loss of generality, assume that when we list the nodes in counterclockwise order starting with the red ones, they are in the order $1,2, \ldots, 2 n$. Let $|R G(\sigma)|$ be the number of red-green pairs in $\sigma$. Define $|G B(\sigma)|$ and $|R B(\sigma)|$ similarly. Assume that $|R G(\sigma)|,|G B(\sigma)|$, and $|R B(\sigma)|$ are all nonzero.

If the set of nodes $\{x, y\}$ contains one red node and one blue node, then $\sigma_{x y}$ has one fewer red-blue pair than $\sigma$, but the number of red-green and green-blue pairs is the same (see Figure 15). By Remark 2.1.18, to determine the relationship between $\operatorname{sign}_{O E}(\sigma)$ and $\operatorname{sign}_{O E}\left(\sigma_{x y}^{\prime}\right)$ it suffices to count the number of nestings in the diagram of $\sigma$ that involve a red-blue pair $\left(n_{r}, n_{b}\right)$. There is one nesting for each red-blue pair other than $\left(n_{r}, n_{b}\right)$, one nesting for each red-green pair, and one nesting for each green-blue pair (see Figure 16). Therefore,

$$
\operatorname{sign}\left(\sigma_{x y}^{\prime}\right)=\operatorname{sign}_{O E}(\sigma) \cdot(-1)^{|R G(\sigma)|} \cdot(-1)^{|G B(\sigma)|} \cdot(-1)^{|R B(\sigma)|-1} .
$$
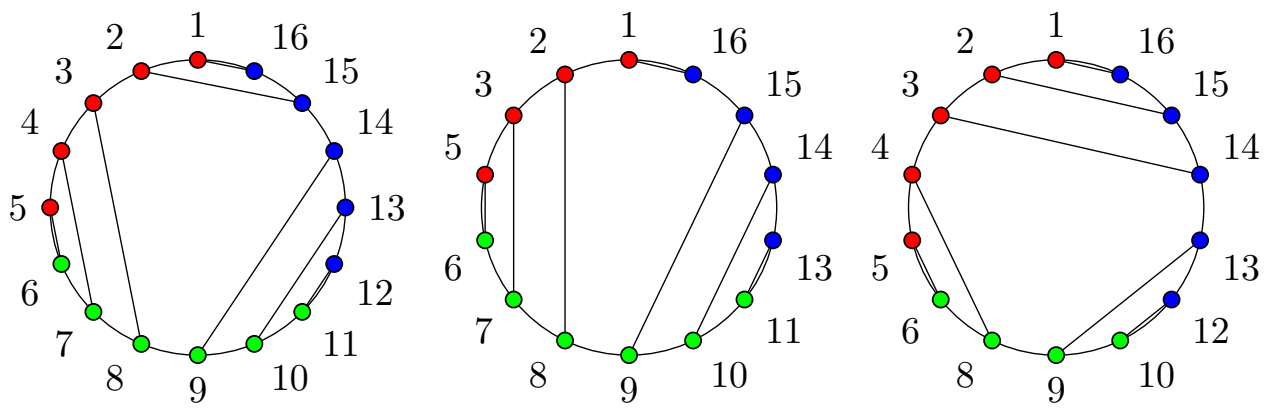

Figure 15. The tripartite pairing on $\mathbf{N}-\{4,12\}$ (shown center) has one fewer red-blue pair than the tripartite pairing on $\mathbf{N}$ (shown left). The tripartite pairing on $\mathbf{N}-\{7,11\}$ (shown right) has one fewer red-green pair, one fewer green-blue pair, and one more red-blue pair compared to the pairing on $\mathbf{N}$.

If the set of nodes $\{x, y\}$ contains one red node and one green node, then $\sigma_{x y}$ has one fewer redgreen pair than $\sigma$, but the number of red-blue and green-blue pairs is the same. So we count the number of nestings in the diagram of $\sigma$ that involve a red-green pair $\left(n_{r}, n_{g}\right)$. There is one nesting for each red-green pair other than $\left(n_{r}, n_{g}\right)$, and one nesting for each red-blue pair. Therefore,

$$
\operatorname{sign}_{O E}\left(\sigma_{x y}^{\prime}\right)=\operatorname{sign}_{O E}(\sigma) \cdot(-1)^{|R B(\sigma)|} \cdot(-1)^{|R G(\sigma)|-1} .
$$

Similarly, if the set of nodes $\{x, y\}$ contains one green node and one blue node, then

$$
\operatorname{sign}_{O E}\left(\sigma_{x y}^{\prime}\right)=\operatorname{sign}_{O E}(\sigma) \cdot(-1)^{|R B(\sigma)|} \cdot(-1)^{|G B(\sigma)|-1} .
$$

If both $x$ and $y$ are green nodes, then $\sigma_{x y}$ has one fewer red-green pair, one fewer green-blue pair, and one more red-blue pair, as shown in Figure 15. Removing a red-green pair from $\sigma$ removes 


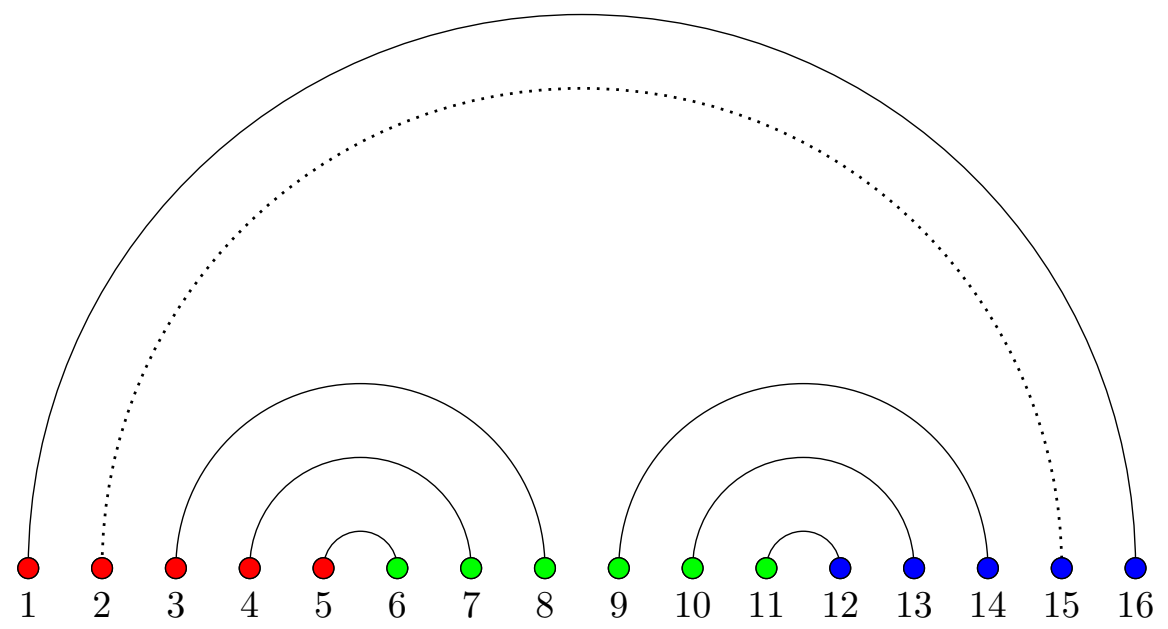

Figure 16. When a red-blue pair is removed from $\sigma$, the number of nestings in the diagram of $\sigma$ decreases by $|R B(\sigma)|-1+|R G(\sigma)|+|G B(\sigma)|$.

$|R B(\sigma)|+|R G(\sigma)|-1$ nestings. Then, removing a green-blue pair removes $|R B(\sigma)|+|G B(\sigma)|-1$ nestings. After these pairs have been removed, adding a red-blue pair results in $|R B(\sigma)|+|G B(\sigma)|-$ $1+|R G(\sigma)|-1$ additional nestings. Therefore,

$$
\operatorname{sign}_{O E}\left(\sigma_{x y}^{\prime}\right)=\operatorname{sign}_{O E}(\sigma) \cdot(-1)^{|R B(\sigma)|} .
$$

If both $x$ and $y$ are red nodes, $\sigma_{x y}$ has one fewer red-blue pair, one fewer red-green pair, and one more green-blue pair. Removing a red-blue pair from $\sigma$ removes $|R G(\sigma)|+|G B(\sigma)|+|R B(\sigma)|-1$ nestings. Then, removing a red-green pair removes $|R B(\sigma)|-1+|R G(\sigma)|-1$ nestings. After these pairs have been removed, adding a green-blue pair results in $|R B(\sigma)|-1+|G B(\sigma)|$ additional nestings. Thus

$$
\operatorname{sign}_{O E}\left(\sigma_{x y}^{\prime}\right)=\operatorname{sign}_{O E}(\sigma) \cdot(-1)^{|R B(\sigma)|} .
$$

Similarly, if both $x$ and $y$ are blue,

$$
\operatorname{sign}_{O E}\left(\sigma_{x y}^{\prime}\right)=\operatorname{sign}_{O E}(\sigma) \cdot(-1)^{|R B(\sigma)|} .
$$

If we assume that the nodes $x, y, w, v$ alternate black and white and the set $\{x, y, w, v\}$ contains at least one node of each RGB color, we can use Remark 3.2.1 to show that the all the signs in Theorem 1.3.3 are positive.

Theorem 1.0.2. Divide the nodes into three circularly contiguous sets $R, G$, and $B$ such that $|R|,|G|$ and $|B|$ satisfy the triangle inequality and let $\sigma$ be the corresponding tripartite pairing. Let $x, y, w, v$ be nodes appearing in a cyclic order such that the set $\{x, y, w, v\}$ contains at least one node of each RGB color. If $x$ and $w$ are both black and $y$ and $v$ are both white, then

$$
\begin{aligned}
Z_{\sigma}^{D D}(G, \mathbf{N}) Z_{\sigma_{x y w v}}^{D D}(G, \mathbf{N}-\{x, y, w, v\})= & Z_{\sigma_{x y} D}^{D D}(G, \mathbf{N}-\{x, y\}) Z_{\sigma_{w v}}^{D D}(G, \mathbf{N}-\{w, v\}) \\
& +Z_{\sigma_{x v}}^{D D}(G, \mathbf{N}-\{x, v\}) Z_{\sigma_{w y}}^{D D}(G, \mathbf{N}-\{w, y\}) .
\end{aligned}
$$

Proof. Without loss of generality, assume that when we list the nodes in counterclockwise order starting with the red ones, they are in the order $1,2, \ldots, 2 n$. Assume also that one of the nodes $x, y, w, v$ is red, two are green, and one is blue. The other cases are very similar. By the assumption that the nodes are in cyclic order, there are two possibilities ${ }^{10}$ :

\footnotetext{
${ }^{10}$ The assumption that the nodes $x, y, w, v$ are in cyclic order is required. Otherwise, it would be possible for $x$ to be red, $y$ to be green, $w$ to be blue, and $v$ to be green. In this case, the sets $\{x, y\}$ and $\{x, v\}$ consist of one red node and one green node, and the sets $\{w, v\}$ and $\{y, w\}$ consists of one green node and one blue node.
} 
(i) One of the sets $\{x, y\},\{w, v\}$ consists of a red node and a green node and the other consists of a green node and a blue node. Also, one of the sets $\{x, v\},\{y, w\}$ consists consists of one red node and one blue node, and the other consists of two green nodes.

(ii) One of the sets $\{x, v\},\{y, w\}$ consists of a red node and a green node and the other consists of a green node and a blue node. Also, one of the sets $\{x, y\},\{w, v\}$ consists consists of one red node and one blue node, and the other consists of two green nodes.

We only prove case (i), as case (ii) is essentially the same. By Remark 3.2.1,

$$
\begin{aligned}
\operatorname{sign}_{O E}\left(\sigma_{x y}^{\prime}\right) \operatorname{sign}_{O E}\left(\sigma_{w v}^{\prime}\right) & =(-1)^{|R B(\sigma)|}(-1)^{|R G(\sigma)|-1}(-1)^{|R B(\sigma)|}(-1)^{|G B(\sigma)|-1} \\
& =(-1)^{|R G(\sigma)|}(-1)^{|G B(\sigma)|}, \text { and } \\
\operatorname{sign}_{O E}\left(\sigma_{x v}^{\prime}\right) \operatorname{sign}_{O E}\left(\sigma_{w y}^{\prime}\right) & =(-1)^{|R G(\sigma)|}(-1)^{|G B(\sigma)|}(-1)^{|R B(\sigma)|-1}(-1)^{|R B(\sigma)|} \\
& =-(-1)^{|R G(\sigma)|}(-1)^{|G B(\sigma)|} .
\end{aligned}
$$

Since we can obtain $\sigma_{x y w v}$ by first removing the nodes $x, y$, and then removing the nodes $w, v$, $\operatorname{sign}_{O E}\left(\sigma_{x y w v}^{\prime}\right)=\operatorname{sign}_{O E}\left(\sigma_{x y}^{\prime}\right) \operatorname{sign}_{O E}\left(\sigma_{w v}^{\prime}\right)$. Thus by Theorem 1.3.3,

$$
\begin{aligned}
Z_{\sigma}^{D D}(G, \mathbf{N}) Z_{\sigma_{x y w v}}^{D D}(G, \mathbf{N}-\{x, y, w, v\})= & Z_{\sigma_{x y} D}^{D D}(G, \mathbf{N}-\{x, y\}) Z_{\sigma_{w v}}^{D D}(G, \mathbf{N}-\{w, v\}) \\
& +Z_{\sigma_{x v}}^{D D}(G, \mathbf{N}-\{x, v\}) Z_{\sigma_{w y}}^{D D}(G, \mathbf{N}-\{w, y\}) .
\end{aligned}
$$

\section{Acknowledgements}

I thank my advisor, Benjamin Young, for posing this problem and his invaluable guidance throughout this project. Thanks also to Richard Kenyon, who provided thoughtful comments on the introduction and to Gregg Musiker for a helpful discussion. Finally, thank you to the anonymous referee who provided numerous helpful comments.

\section{REFERENCES}

[Bri11] Tom Bridgeland. Hall algebras and curve-counting invariants. J. Amer. Math. Soc., 24(4):969-998, 2011.

[DFGG97] P. Di Francesco, O. Golinelli, and E. Guitter. Meanders and the Temperley-Lieb algebra. Commun. Math. Phys., 186(1):1-59, 1997.

[Dub19] Julien Dubédat. Double dimers, conformal loop ensembles and isomonodromic deformations. J. Eur. Math. Soc. (JEMS), 21(1):1-54, 2019.

[FZ02] Sergey Fomin and Andrei Zelevinsky. Cluster algebras. I. Foundations. J. Amer. Math. Soc., 15(2):497529, 2002.

[GR19] Nahid Ghodratipour and Shahin Rouhani. Connection probabilities in the double-dimer model-the case of two connectivity patterns. arXiv preprint arXiv:1908.07595, 2019.

[JWY20] Helen Jenne, Gautam Webb, and Benjamin Young. The combinatorial PT-DT correspondence. arXiv preprint arXiv:2012.08484, 2020.

[Kas67] P. W. Kasteleyn. Graph theory and crystal physics. In Graph Theory and Theoretical Physics, pages 43-110. Academic Press, London, 1967.

[Ken14] Richard Kenyon. Conformal invariance of loops in the double-dimer model. Comm. Math. Phys., 326(2):477-497, 2014.

[KP16] Richard Kenyon and Robin Pemantle. Double-dimers, the Ising model and the hexahedron recurrence. J. Combin. Theory Ser. A, 137:27-63, 2016.

[Kuo04] Eric H. Kuo. Applications of graphical condensation for enumerating matchings and tilings. Theoret. Comput. Sci., 319(1-3):29-57, 2004.

[Kup94] Greg Kuperberg. Symmetries of plane partitions and the permanent-determinant method. J. Comb. Theory, 68:115-151, 1994.

[KW09] Richard W. Kenyon and David B. Wilson. Combinatorics of tripartite boundary connections for trees and dimers. Electron. J. Comb., 16(1), 2009.

[KW11a] Richard W. Kenyon and David B. Wilson. Boundary partitions in trees and dimers. Trans. Amer. Math. Soc., 363(3):1325-1364, 2011. 
[KW11b] Richard W. Kenyon and David B. Wilson. Double-dimer pairings and skew Young diagrams. Electron. J. Combin., 18(1):Paper 130, 22, 2011.

[LM17] Tri Lai and Gregg Musiker. Beyond Aztec castles: Toric cascades in the dP $P_{3}$ quiver. Comm. Math. Phys., 356(3):823-881, 2017.

[LM20] Tri Lai and Gregg Musiker. Dungeons and Dragons: Combinatorics for the dP $P_{3}$ quiver. Ann. Comb., 24(2):257-309, 2020.

[Mac16] Percy A. MacMahon. Combinatory analysis. Cambridge University Press, The Edinburgh Building, Cambridge, UK, 1915-16.

[MNOP06a] D. Maulik, N. Nekrasov, A. Okounkov, and R. Pandharipande. Gromov-Witten theory and DonaldsonThomas theory, I. Compos. Math., 142(5):1263-1285, 2006.

[MNOP06b] D. Maulik, N. Nekrasov, A. Okounkov, and R. Pandharipande. Gromov-Witten theory and DonaldsonThomas theory, II. Compos. Math., 142(5):1286-1304, 2006.

[MOOP11] D. Maulik, A. Oblomkov, A. Okounkov, and R. Pandharipande. Gromov-Witten/Donaldson-Thomas correspondence for toric 3-folds. Invent. Math., 186(2):435-479, 2011.

[PT09a] Rahul Pandharipande and Richard P. Thomas. The 3-fold vertex via stable pairs. Geom. Topol., 13(4):1835-1876, 2009.

[PT09b] Rahul Pandharipande and Richard P. Thomas. Curve counting via stable pairs in the derived category. Invent. Math., 178(2):407-447, 2009.

[Tod10] Yukinobu Toda. Curve counting theories via stable objects I. DT/PTcorrespondence. J. Amer. Math. Soc., 23(4):1119-1157, 2010.

[Wil14] Lauren K. Williams. Cluster algebras: an introduction. Bull. Amer. Math. Soc. (N.S.), 51(1):1-26, 2014. 